\title{
Analysis of the Stick-Slip Problem for Non-Newtonian Flows
}

\author{
Marco A. Fontelos \\ Institute for Mathematics and its Applications, University of Minnesota, \\ Minneapolis, Minnesota 55455. \\ Avner Friedman \\ University of Minnesota, Department of Mathematics, \\ Minneapolis, Minnesota 55410.
}

August 1, 1999

\begin{abstract}
In this paper we consider the stick-slip problem for non-Newtonian flows: the fluid emerges from a 2-dimensional bounded strip $\{(x, y)$; $-N<x<0,|y|<1\}$ into the space $\{0<x<N\}$ and its free boundary is linearized to $\{x>0 ;|y|=1\}$; here for simplicity $N$ is a finite number which can be taken, however, arbitrarily large. In Part 1 we formulate the problem for a class of Oldroyd models under certain conditions on its parameters and then transform it, by means of integration along streamlines, in such a way that the solution is identified with a fixed point of a mapping $W$. In order to analyze the properties of $W$ we first solve, in Part 2, a hyperbolic system along streamlines; the analysis is most delicate near the points $(0, \pm 1)$ where the stick and slip boundaries meet. Next, in Part 3, we solve an elliptic problem for the biharmonic operator in the strip, with boundary conditions that change abruptly at $(0, \pm 1)$. Finally, on the basis of the results or Parts 2 and 3, we establish in Part 4 the existence and uniqueness of a fixed point for $W$. The singularity of the solution at $(0, \pm 1)$, to a leading order, is Newtonian; the next order term, however, is non-Newtonian: it is highly oscillating near the slip boundary. In Part 4 we also show in a formal way that, if the conditions on the parameters (mentioned above) are not satisfied, then the Oldroyd-modelled flow develops, to a leading order, a non-Newtonian singularity at $(0, \pm 1)$ in a boundary layer near $y= \pm 1$, and we derive the explicit structure of this singularity in some cases.
\end{abstract}




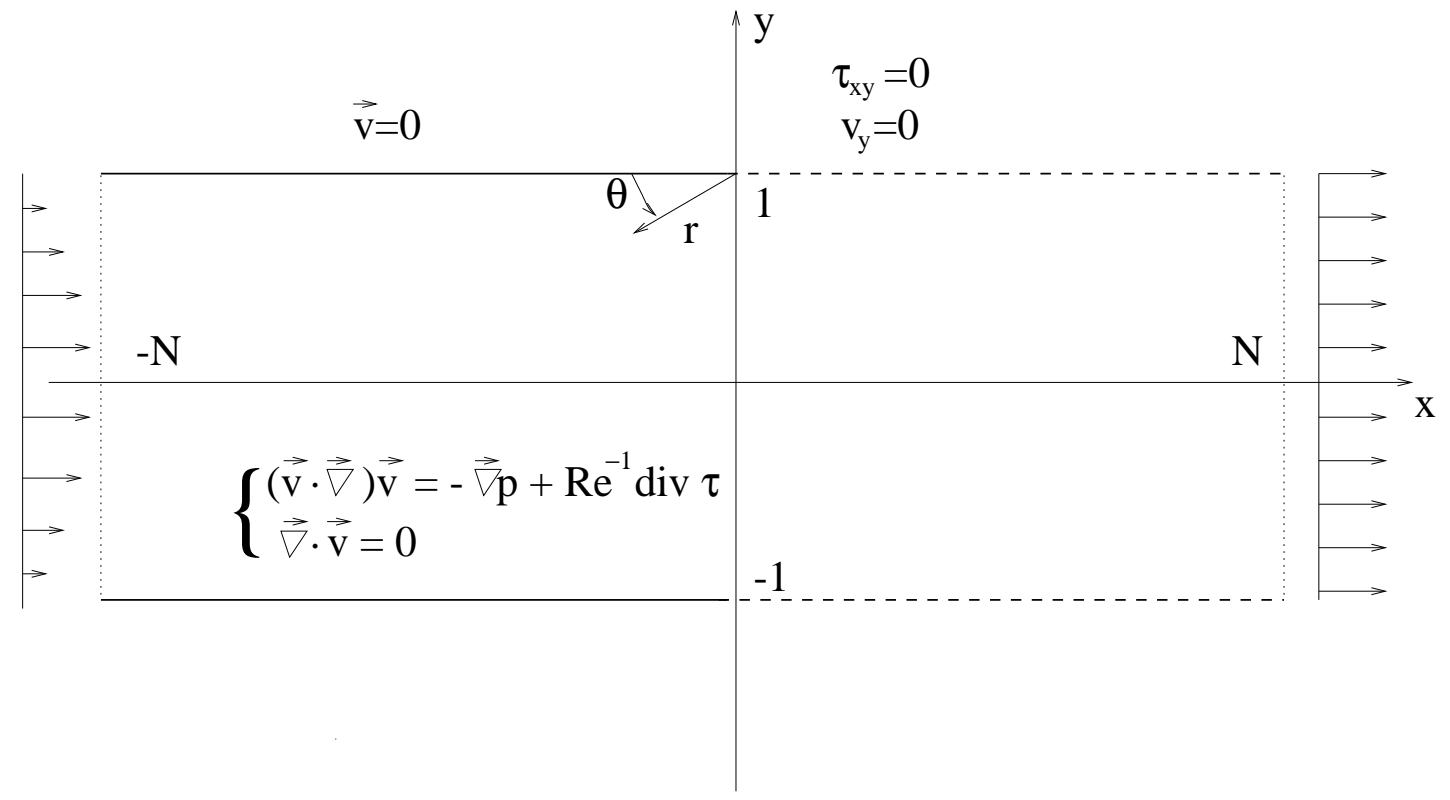

Figure 1: The stick-slip problem

\section{Part I}

\section{Formulation of the problem and outline of its solution}

\section{Introduction}

The die-swell problem models either a 3-dimensional axially symmetric jet flow from a pipe or its 2-dimensional counterpart (symmetric planar flow). Its linearized version is called the stick-slip problem (see Figure 1). Both problems have been studied for Newtonian flows. The stick-slip problem for Stokes flow (Newtonian fluid without inertia) was completely solved by Richardson (cf. [12]) in the planar case and by Trogdon and Joseph (cf. [16], [17]) in the 3-d axisymmetric case, while the die-swell problem has been solved very recently by Solonnikov (cf. [15]). For non-Newtonian fluids, the question of well-posedness of these problems remains open. Numerical simulations (see for example [1], [13], [14] and the references contained there) suggest that for some viscoelastic models, such as Maxwell or Oldroyd-B models, non-integrable singularities in the stresses do appear. Some authors have proposed several alternative models which would yield integrable Newtonian-like stresses. One of these is the modified upper convected Maxwell model (MUCM) which introduces a dependence of the relaxation time on the trace of the stress tensor (see [1]). Another model allows some slippage at the solid walls in order to alleviate the otherwise strongly singular stresses (see [14]). 
In this paper we study the stick-slip problem for some non-Newtonian flows in a strip

$$
\Sigma=\{(x, y) ;-N<x<N,-1<y<1\}
$$

and establish its well posedness; our results can probably be extended to $N=$ $\infty$, but the assumption that $N$ is finite simplifies the analysis.

The fluid is assumed to satisfy the conservation of momentum and mass equations:

$$
\begin{aligned}
(\vec{v} \cdot \nabla) \vec{v} & =-\nabla p+\frac{1}{\operatorname{Re}} \operatorname{div} \tau \text { in } \Sigma \\
\nabla \cdot \vec{v} & =0 \quad \text { in } \Sigma
\end{aligned}
$$

where $\vec{v}=\left(v_{1}, v_{2}\right)$, Re is the Reynolds number and $\tau$ satisfies a constitutive equation. For Newtonian fluids, the constitutive relation is simply

$$
\boldsymbol{\tau}=\mathbf{D}
$$

or, in components,

$$
\tau_{i j}=D_{i j} \equiv \frac{1}{2}\left(\frac{\partial v_{i}}{\partial x_{j}}+\frac{\partial v_{j}}{\partial x_{i}}\right),
$$

while for non-Newtonian fluids the relation between the tensors $\boldsymbol{\tau}$ and $\mathbf{D}$ is usually non-linear and involves derivatives of both tensors. We focus our attention on Oldroyd models in the Johnson-Segalman form. These models are characterized by a constitutive relation (see [5]):

$$
\boldsymbol{\tau}+\alpha_{1}\left(\boldsymbol{\tau}_{(1)}+c(\operatorname{Tr} \boldsymbol{\tau}) \mathbf{D}+d(\boldsymbol{\tau}: \mathbf{D}) \mathbf{I}\right)=\mathbf{D}+\alpha_{2}\left(\mathbf{D}_{(1)}+d(\mathbf{D}: \mathbf{D}) \mathbf{I}\right)
$$

where

$$
\boldsymbol{\tau}: \mathbf{D}=\sum_{i, j} \tau_{i j} D_{i j}, \mathbf{D}: \mathbf{D}=\sum_{i, j} D_{i j} D_{i j}
$$

$c$ and $d$ are any real parameters and $\alpha_{1}$ and $\alpha_{2}$ are positive constants, called "relaxation times", and $\boldsymbol{\tau}_{(1)}, \mathbf{D}_{(1)}$ are tensorial material derivatives; note that (by (1.2)) $\operatorname{Tr} \mathbf{D}=0$. An invariance argument shows that the general form of $\boldsymbol{\tau}_{(1)}$ and $\mathbf{D}_{(1)}$ are (cf [5]; Chap. 1):

$$
\begin{aligned}
\boldsymbol{\tau}_{(1)} & =(\vec{v} \cdot \nabla) \boldsymbol{\tau}-\mathbf{W} \boldsymbol{\tau}+\boldsymbol{\tau} \mathbf{W}-a(\mathbf{D} \boldsymbol{\tau}+\boldsymbol{\tau} \mathbf{D}) \\
\mathbf{D}_{(1)} & =(\vec{v} \cdot \nabla) \mathbf{D}-\mathbf{W D}+\mathbf{D W}-2 a \mathbf{D D},
\end{aligned}
$$

where

$$
\begin{gathered}
\mathbf{W}=\frac{1}{2}\left(\nabla \vec{v}-(\nabla \vec{v})^{T}\right), \\
\nabla \vec{v}=\left(\begin{array}{cc}
\frac{\partial v_{x}}{\partial x} & \frac{\partial v_{x}}{\partial y} \\
\frac{\partial v_{y}}{\partial x} & \frac{\partial v_{y}}{\partial y}
\end{array}\right), \quad\left(v_{x}=v_{1}, v_{y}=v_{2}\right),
\end{gathered}
$$

and $a$ is a real parameter; note that

$$
\mathbf{D}=\frac{1}{2}\left(\nabla \vec{v}+(\nabla \vec{v})^{T}\right) .
$$


The expression in (1.4) for $\boldsymbol{\tau}_{(1)}$ (or $\mathbf{D}_{(1)}$ ) is called the upper convected derivative when $a=1$, the lower convected derivative when $a=-1$, and the corotational or Jaumann derivative when $a=0$. If $c=0$ then the constitutive law (1.3) corresponds to an Oldroyd interpolated model.

We shall be looking for solutions

$$
\boldsymbol{\tau}=\left(\begin{array}{cc}
\tau_{x x} & \tau_{x y} \\
\tau_{y x} & \tau_{y y}
\end{array}\right)
$$

of (1.1), (1.2) for which $\tau_{x y}=\tau_{y x}$; this is compatible with the fact that equation (1.4) is identical to its transpose.

The non-Newtonian law (1.3) with $\boldsymbol{\tau}_{(1)}$ and $\mathbf{D}_{(1)}$ defined by (1.4) presents serious difficulties for both analysis and computation of the flow near the initial points of the free boundary, i.e., near the points $(0, \pm 1)$. Following other authors who have done numerical computations (see [1], [13], [14]) and asymptotic analysis (see [11]), we shall replace (in all but the last section of this paper) $\vec{v}$ in the definitions of $\boldsymbol{\tau}_{(1)}$ and $\mathbf{D}_{(1)}$ by a prescribed Newtonian velocity $\vec{v}_{0}$. Thus we shall work with the non-Newtonian law:

$$
\boldsymbol{\tau}+\alpha_{1}\left(\boldsymbol{\tau}_{(1)}+c(\operatorname{Tr} \boldsymbol{\tau}) \mathbf{D}_{0}+d\left(\boldsymbol{\tau}: \mathbf{D}_{0}\right) \mathbf{I}\right)=\mathbf{D}+\alpha_{2}\left(\mathbf{D}_{(1)}+d\left(\mathbf{D}: \mathbf{D}_{0}\right) \mathbf{I}\right)
$$

where

$$
\begin{aligned}
\boldsymbol{\tau}_{(1)} & =\left(\vec{v}_{0} \cdot \nabla\right) \boldsymbol{\tau}-\mathbf{W}_{0} \boldsymbol{\tau}+\boldsymbol{\tau} \mathbf{W}_{0}-a\left(\mathbf{D}_{0} \boldsymbol{\tau}+\boldsymbol{\tau} \mathbf{D}_{0}\right) \\
\mathbf{D}_{(1)} & =\left(\vec{v}_{0} \cdot \nabla\right) \boldsymbol{\tau}-\mathbf{W}_{0} \mathbf{D}+\mathbf{D} \mathbf{W}_{0}-a\left(\mathbf{D}_{0} \mathbf{D}+\mathbf{D D}_{0}\right)
\end{aligned}
$$

and

$$
\begin{aligned}
\mathbf{W}_{0} & =\frac{1}{2}\left(\nabla \vec{v}_{0}-\left(\nabla \vec{v}_{0}\right)^{T}\right), \\
\mathbf{D}_{0} & =\frac{1}{2}\left(\nabla \vec{v}_{0}+\left(\vec{v}_{0}\right)^{T}\right), \\
\nabla \vec{v}_{0} & =\left(\begin{array}{ll}
\frac{\partial v_{0, x}}{\partial x} & \frac{\partial v_{0, x}}{\partial y} \\
\frac{\partial v_{0, y}}{\partial x} & \frac{\partial v_{0, y}}{\partial y}
\end{array}\right) .
\end{aligned}
$$

The Newtonian velocity field $\overrightarrow{v_{0}}$ will be a flow similar to that computed by Richardson [12] for the Newtonian stick-slip problem in a strip; a precise set of conditions on $\overrightarrow{v_{0}}$ will be given in Definition 2.2.

The boundary conditions are no-slip at the solid surfaces

$$
\vec{v}=0 \text { at } \Gamma_{0}=\{(x, y) ;-N<x<0, y= \pm 1\}
$$

and, at the fluid lines, $\Gamma_{1}$ :

$$
\begin{gathered}
\vec{v} \cdot \vec{n}=0 \quad \text { at } \quad \Gamma_{1}=\{(x, y) ; 0<x<N, y= \pm 1\} \\
\tau_{x y}=0 \quad \text { at } \Gamma_{1}=\{(x, y) ; 0<x<N, y= \pm 1\} .
\end{gathered}
$$

Condition (1.12) means that $\Gamma_{1}$ is a stream line, while Condition (1.13) means that no shear stress is exerted by the fluid at $\Gamma_{1}$. These two conditions form 
the linearized version of the full boundary conditions in the die-swell problem when the free boundary problem is approximated by $\Gamma_{1}$.

We shall also impose flux conditions at $x= \pm N$ :

$$
\begin{aligned}
& \vec{v}=\vec{v}_{1}(y)=\left(g_{1}(y), 0\right) \text { at } x=-N, \\
& \vec{v}=\vec{v}_{2}(y)=\left(g_{2}(y), 0\right) \text { at } x=N
\end{aligned}
$$

with the constraint

$$
\int_{-1}^{1} g_{1}(y) d y=\int_{-1}^{1} g_{2}(y) d y
$$

and the compatibility condition

$$
\vec{v}_{1}( \pm 1)=0
$$

The constraint (1.16) follows by conservation of mass, and this condition together with (1.11) assure that $\vec{v}$ is continuous at $(-N, \pm 1)$.

We shall later on prescribe values for $g_{1}(y), g_{2}(y)$ in a rather natural way.

In this paper, we present a systematic analysis of the models of the type just described under some assumptions on the parameters $a, c$ and $d$. The behavior of the non-Newtonian flow will depend, in particular, on the parameters

$$
\begin{aligned}
b & =\frac{3 d a-2 a^{2}-3 d c+2 a c}{1-d a+d c+a^{2}-a c}, \\
b^{\prime} & =2 c a-6 c d-4 a^{2}+12 a d-4 d^{2}
\end{aligned}
$$

note that if $d=a$ then $b=a^{2}-a c$ and $b^{\prime}=4\left(a^{2}-a c\right)=4 b$. Most of the literature is concentrated on the so called Oldroyd A and B models, for which $a= \pm 1, c=d=0$. When $a=c=d=0$, the model we obtain is called Jeffrey's model and may be considered equivalent to Oldroyd A and B models with a corotational derivative instead of upper or lower convected derivatives (cf. [5] or the chapters 7 and 8 of [2] for an extensive description of the models based on a corotational derivative). In all but the last section of the paper we assume that $d=a$, and $b=a^{2}-a c$ lies in the interval $\left(0, \frac{3}{2}\right)$. We establish existence and uniqueness of the flow, and derive a genuinely non-Newtonian feature, namely:

Near the points $(0, \pm 1)$ there appears, in addition to a leading order Newtonian singularity, a highly oscillatory lower order term.

Our method extends to Jeffrey's model, which is briefly discussed in Section 13. In this case the highest order singularity in non-Newtonian:

$$
\psi_{y y} \sim C \log \frac{1-|y|}{x} \text { as }|y| \rightarrow 1,1-|y|<<x^{\frac{3}{2}}, x>0
$$

where $\psi$ is the stream function, whereas for Newtonian flow $\psi_{y y}$ is bounded in the same region.

In the last section of the paper (Section 14) we drop the condition $d=a$ and, by formal arguments based on the analysis done in previous sections, derive the following result:

The flow develops a non-Newtonian singularity within a neighborhood of $(0, \pm 1)$, in a boundary layer near to the free boundary, where very high stresses 
are concentrated (the stresses are nevertheless integrable). The non-Newtonian singularity can be expressed in the form

$$
\psi_{y y} \sim C\left(\frac{1}{(1-|y|)^{\frac{2}{3}} x^{\frac{1}{3}}}\right)^{\frac{2}{5} b} \text { as }|y| \rightarrow 1,1-|y|<<x^{\frac{\frac{3}{2}-\frac{2}{15} b}{1+\frac{4}{15} b}}, x>0
$$

This formal result is consistent with observations of authors that have performed numerical experiments (cf. [1], [13], [14] and the references mentioned there, and [11] for results on a related problem). Indeed these experiments show the appearance of regions close to the free boundary in a neighborhood of $(0, \pm 1)$ where very high stresses concentrate, as well as the onset of very strong oscillations.

Throughout Sections 1-12 we assume that:

$$
\begin{aligned}
& d=a \\
& 0<b \equiv a^{2}-c a<\frac{3}{2}
\end{aligned}
$$

Re and the absolute value of $\epsilon \equiv 1-\frac{\alpha_{2}}{\alpha_{1}}$ are small.

We note that the smallness of $|\epsilon|$ and Re do not depend on how large $N$ is.

Definition 1.1 The problem of solving the system consisting of the differential equations (1.1), (1.2) with the non-Newtonian constitutive laws (1.5)-(1.10) and the boundary conditions (1.11)-(1.15) subject to (1.16), (1.17) and (1.22)(1.24) will be called Problem (A).

In the next section we reformulate Problem (A) in a more convenient form which will enable us to reduce it to a statement that a linear mapping $W$ has a fixed point. $W$ is defined by solving a hyperbolic system in the strip $\Sigma$, followed by solving an elliptic problem for the biharmonic operator, with boundary conditions that change abruptly at $(0, \pm 1)$. The hyperbolic system is analyzed in Part 2, and the elliptic problem is analyzed in Part 3. Finally, in Part 4 we prove that $W$ has a fixed point, and, in the last section, we consider the case $d \neq a$. Section 3 (Part 1) contains some results (needed in Part 2) on a linear system of differential equations along characteristics.

\section{Reformulation of Problem (A)}

If we substitute from (1.6)-(1.10) into (1.5) and use the facts that $\operatorname{div} \overrightarrow{v_{0}}=$ $\frac{\partial v_{0, x}}{\partial x}+\frac{\partial v_{0, y}}{\partial y}=0, \tau_{x y}=\tau_{y x}$ and $\operatorname{Tr} \mathbf{D}=0$ we obtain, in matrix notation,

$$
\begin{aligned}
& \left(\begin{array}{c}
\tau_{x x} \\
\tau_{y y} \\
\tau_{x y}
\end{array}\right)+\alpha_{1}\left((\vec{v} \cdot \nabla)\left(\begin{array}{c}
\tau_{x x} \\
\tau_{y y} \\
\tau_{x y}
\end{array}\right)+A\left(\begin{array}{c}
\tau_{x x} \\
\tau_{y y} \\
\tau_{x y}
\end{array}\right)\right) \\
= & \left(\begin{array}{c}
D_{x x} \\
D_{y y} \\
D_{x y}
\end{array}\right)+\alpha_{2}\left((\vec{v} \cdot \nabla)\left(\begin{array}{c}
D_{x x} \\
D_{y y} \\
D_{x y}
\end{array}\right)+A\left(\begin{array}{c}
D_{x x} \\
D_{y y} \\
D_{x y}
\end{array}\right)\right)
\end{aligned}
$$


where

$$
\begin{gathered}
A=A\left(\vec{v}_{0}\right)= \\
\left(\begin{array}{cc}
(-2 a+c+2 d) \frac{\partial v_{0, x}}{\partial x} & (c-2 d) \frac{\partial v_{0, x}}{\partial x} \\
(-c+2 d) \frac{\partial v_{0, x}}{\partial x} & (2 a-c-2 d) \frac{\partial v_{0, x}}{\partial x} \\
-\frac{1+a-c}{2} \frac{\partial v_{0, y}}{\partial x}+\frac{1-a+c}{2} \frac{\partial v_{0, x}}{\partial y} & \frac{1-a+c}{2} \frac{\partial v_{0, y}}{\partial x}-\frac{1+a-c}{2} \frac{\partial v_{0, x}}{\partial y} \\
\frac{\partial v_{0, y}}{\partial x}-\frac{\partial v_{0, x}}{\partial y}+(d-a)\left(\frac{\partial v_{0, y}}{\partial x}+\frac{\partial v_{0, x}}{\partial y}\right) \\
-\frac{\partial v_{0, y}}{\partial x}+\frac{\partial v_{0, x}}{\partial y}+(d-a)\left(\frac{\partial v_{0, y}}{\partial x}+\frac{\partial v_{0, x}}{\partial y}\right) \\
0
\end{array}\right)
\end{gathered}
$$

Using the assumption $d=a$, the matrix $A\left(\vec{v}_{0}\right)$ simplifies to

$$
\begin{gathered}
A=A\left(\vec{v}_{0}\right) \\
=\left(\begin{array}{ccc}
c \frac{\partial v_{0, x}}{\partial x} & (c-2 a) \frac{\partial v_{0, x}}{\partial x} & \frac{\partial v_{0, y}}{\partial x}-\frac{\partial v_{0, x}}{\partial y} \\
(-c+2 a) \frac{\partial v_{0, x}}{\partial x} & -c \frac{\partial v_{0, x}}{\partial x} & -\frac{\partial v_{0, y}}{\partial x}+\frac{\partial v_{0, x}}{\partial y} \\
-\frac{1+a-c}{2} \frac{\partial v_{0, y}}{\partial x}+\frac{1-a+c}{2} \frac{\partial v_{0, x}}{\partial y} & \frac{1-a+c}{2} \frac{\partial v_{0, y}}{\partial x}-\frac{1+a-c}{2} \frac{\partial v_{0, x}}{\partial y} & 0
\end{array}\right)
\end{gathered}
$$

Introducing the differential operator

$$
K T \equiv T+\alpha_{1}\left(\left(\vec{v}_{0} \cdot \nabla\right) T+A\left(\vec{v}_{0}\right) T\right),
$$

we can rewrite $(2.1)$ in the form

$$
K T=\epsilon D
$$

where

$$
T=\left(\begin{array}{c}
T_{x x} \\
T_{y y} \\
T_{x y}
\end{array}\right)=\left(\begin{array}{c}
\tau_{x x} \\
\tau_{y y} \\
\tau_{x y}
\end{array}\right)-\frac{\alpha_{2}}{\alpha_{1}}\left(\begin{array}{c}
D_{x x} \\
D_{y y} \\
D_{x y}
\end{array}\right) \equiv \tau-\frac{\alpha_{2}}{\alpha_{1}} D
$$

and $\epsilon=1-\frac{\alpha_{2}}{\alpha_{1}}$ (see (1.24)); note that $T_{x y}=T_{y x}$. The idea of formulating the hyperbolic problem associated to the constitutive equations in the form (2.5) was introduced in [11] (see also [3]).

Equation (2.4) has to be complemented by prescribing a boundary condition on $T$ at $x=-N$. Since we wish to approximate the solution of the stick-slip problem in the entire strip $\{-1<y<1\}$, a natural choice for $T$ is its formal limit when $N \rightarrow-\infty$. This leads to:

$$
\begin{gathered}
T_{x x}(-N, y)=\frac{1}{2} \alpha_{1} \epsilon \frac{g_{1}^{\prime 2}}{1+\alpha_{1}^{2} g_{1}^{\prime 2}} \\
T_{y y}(-N, y)=-\frac{1}{2} \alpha_{1} \epsilon \frac{g_{1}^{\prime 2}}{1+\alpha_{1}^{2} g_{1}^{\prime 2}} \\
T_{x y}(-N, y)=\frac{1}{2} \epsilon \frac{1}{1+\alpha_{1}^{2} g_{1}^{\prime 2}}
\end{gathered}
$$

where $g_{1}$ satisfies the differential equation

$$
\frac{1}{2}\left(\frac{\alpha_{2}}{\alpha_{1}} g_{1}^{\prime}+\left(1-\frac{\alpha_{2}}{\alpha_{1}}\right) \frac{g_{1}^{\prime}}{1+(c-a) \alpha_{1}^{2} g^{\prime 2}}\right)=-y
$$


with $g_{1}( \pm 1)=0$ (by (1.17)).

Indeed, if $\vec{v}=\vec{v}(y)$ (as $x \rightarrow-\infty)$, then (1.2) implies that

$$
\vec{v}=\left(g_{1}(y), 0\right)
$$

and (1.1) reduces to

$$
-\nabla p+\frac{1}{\operatorname{Re}} \operatorname{div} \boldsymbol{\tau}=0 .
$$

We also have $g_{1}( \pm 1)=0$. A simple computation shows that (1.4) reduces to

$$
=g_{1}^{\prime}\left(\begin{array}{cc}
\boldsymbol{\tau}_{(1)} \\
-\tau_{x y} \\
\frac{1}{2}\left((1-a+c) \tau_{y y}-(1+a-c) \tau_{x x}\right)
\end{array}\right)
$$

and, similarly,

$$
\mathbf{D}_{(1)}=-g_{1}^{\prime}\left(\begin{array}{cc}
\frac{1}{2} g_{1}^{\prime} & 0 \\
0 & \frac{1}{2} g_{1}^{\prime}
\end{array}\right)
$$

so that the system (1.3) becomes

$$
\begin{aligned}
\tau_{x x}-\alpha_{1} g_{1}^{\prime} \tau_{x y} & =-\frac{\alpha_{2}}{2} g_{1}^{\prime 2}, \\
\tau_{x y}+\frac{\alpha_{1}}{2} g_{1}^{\prime}\left((1-a+c) \tau_{y y}-(a+1-c) \tau_{x x}\right) & =\frac{1}{2} g_{1}^{\prime} \\
\tau_{y y}-\alpha_{1} g_{1}^{\prime} \tau_{x y} & =-\frac{\alpha_{2}}{2} g_{1}^{\prime 2} .
\end{aligned}
$$

We easily find that

$$
\begin{aligned}
\tau_{x x} & =\frac{1}{2} \alpha_{1}\left(1-\frac{\alpha_{2}}{\alpha_{1}}\right) \frac{g_{1}^{\prime 2}}{1+(1+c-a) \alpha_{1}^{2} g_{1}^{\prime 2}}, \\
\tau_{y y} & =\frac{1}{2} \alpha_{1}\left(1-\frac{\alpha_{2}}{\alpha_{1}}\right) \frac{g_{1}^{\prime 2}}{1+(1+c-a) \alpha_{1}^{2} g_{1}^{\prime 2}},
\end{aligned}
$$

and

$$
\tau_{x y}=\frac{1}{2}\left(\frac{\alpha_{2}}{\alpha_{1}} g_{1}^{\prime}+\left(1-\frac{\alpha_{2}}{\alpha_{1}}\right) \frac{g_{1}^{\prime}}{1+(1+c-a) \alpha_{1}^{2} g_{1}^{\prime 2}}\right) .
$$

Taking the curl (defined by $\left.\operatorname{curl} \vec{w}=\operatorname{curl}\left(w_{1}, w_{2}\right)=\frac{\partial w_{1}}{\partial y}-\frac{\partial w_{2}}{\partial x}\right)$ of (2.10) and using the fact that $\tau_{x x}, \tau_{y y}, \tau_{x y}$ do not depend on $x$ (as $x \rightarrow-\infty$ ), we obtain

$$
\frac{d^{2} \tau_{x y}}{d y^{2}}=0 \quad \text { at } x=-\infty
$$

Comparing this with equation (2.13) and assuming that $g_{1}(y)$ is symmetric in $y$, yields equation (2.8).

Remark 2.1. The above analysis can also be extended to the case $x=+\infty$ and it shows that $g_{2}(y)=$ constant. By (1.16) we then have

$$
g_{2}=\frac{1}{2} \int_{-1}^{1} g_{1}(y) d y
$$


Since $\vec{v}_{0}$ is a vector field in $\Sigma$ which describes a flow that enters $\Sigma$ at $x=-N$ and does not leave $\Sigma$ except at the boundary $x=N$, one should be able, in principle, to solve (2.4) for $T$ (in terms of $D$ ) subject to the conditions (2.7) at $x=-N$. We shall denote this solution by $\epsilon K^{-1} D$, or, in matrix form

$$
\mathbf{T}=\left(\begin{array}{cc}
T_{x x} & T_{x y} \\
T_{y x} & T_{y y}
\end{array}\right) \equiv \epsilon \mathbf{K}^{-1} \mathbf{D}
$$

Applying the curl operator to the conservation of momentum equation (1.1) we get

$$
\operatorname{Re} \operatorname{curl}(\vec{v} \cdot \nabla) \vec{v}=\operatorname{curl} \operatorname{div}(\tau) .
$$

By (1.2), there exists a stream function $\psi$ such that

$$
v_{x}=\psi_{y}, \quad v_{y}=-\psi_{x}
$$

From the definition of $T$ in (2.6) and (2.17), (2.16), we then have,

$$
\operatorname{Re} \operatorname{curl}(\vec{v} \cdot \nabla) \vec{v}=\operatorname{curl} \operatorname{div}\left(\mathbf{T}+\frac{\alpha_{2}}{\alpha_{1}} \mathbf{D}\right)=\frac{\alpha_{2}}{\alpha_{1}} \Delta^{2} \psi+\operatorname{curl} \operatorname{div}\left(\epsilon \mathbf{K}^{-1} \mathbf{D}\right) .
$$

Thus the stream function $\psi$ satisfies the equation

$$
\Delta^{2} \psi=\frac{\alpha_{1}}{\alpha_{2}} \operatorname{Re} \operatorname{curl}(\vec{v} \cdot \nabla) \vec{v}+\frac{\epsilon}{\epsilon-1} \operatorname{curldiv}\left(\mathbf{K}^{-1} \mathbf{D}\right) \text { in } \Sigma .
$$

From the boundary conditions (1.11), (1.12), we derive the following conditions for $\psi$ :

$$
\begin{aligned}
& \psi=\psi_{y}=0 \quad \text { at } \Gamma_{0}=\{(x, y) ;-N<x<0, y= \pm 1\} \\
& \psi=0 \quad \text { at } \Gamma_{1}=\{(x, y) ; 0<x<N, y= \pm 1\}
\end{aligned}
$$

Taking into account (2.6) and (1.24), the boundary condition $\tau_{x y}=0$ (see (1.13)) may be written as

$$
D_{x y}=\frac{1}{\epsilon-1} T_{x y} \text { on } \Gamma_{1}=\{(x, y) ; 0<x<N, y= \pm 1\} .
$$

Note that the last condition on $\vec{v}$ can be written, in terms of $\psi$, as $\psi_{y y}-$ $\psi_{x x}=\frac{1}{\epsilon-1} T_{x y}$, or, by $(2.20)$, as

$$
\psi_{y y}=-\frac{1}{1-\epsilon} T_{x y} \text { on } \Gamma_{1}=\{(x, y) ; 0<x<N, y= \pm 1\}
$$

Definition 2.1 The problem of solving the differential equation (2.18) with the boundary conditions (2.19),(2.20), (2.21) and (1.14), (1.15) ( $g_{1}$ is defined by (2.8) with $g_{1}( \pm 1)=0$ and $g_{2}$ is a constant satisfying (2.15)) with $\vec{v}=$ $\left(\psi_{y},-\psi_{x}\right)$ will be called Problem $(L)$.

Having reduced Problem (A) to Problem (L), we shall henceforth concentrate on the latter problem.

In order to complete the formulation of Problem (L) (or (A)) we need to impose precise conditions on $\vec{v}_{0}$. 
Definition 2.2 A vector field $\vec{v}=\left(v_{x}, v_{y}\right)$ defined in $\bar{\Sigma}$ is said to belong to class $V_{0}$ if

(i) $\vec{v}$ is in $C^{1+\alpha}(\bar{\Sigma})$;

(ii) $\vec{v}$ describes a flow that enters $\Sigma$ from $x=-N$, does not leave $\Sigma$ at $y= \pm 1$, and exits $\Sigma$ at $x=N$, and moreover, $v_{x}( \pm N, y)>0$ for $|y|<1$;

(iii) there exists a function $\psi$ such that $\vec{v}=\left(\psi_{y},-\psi_{x}\right)$ with $\nabla \psi \neq 0$ in $\Sigma$, and the characteristic curves defined as the solutions of

$$
\frac{d x}{d s}=\psi_{y}, \frac{d y}{d s}=-\psi_{x}
$$

passing through a point in $\Sigma$ remain in $\Sigma$ as long as $|x|<N$.

(iv) $\psi$ satisfies the boundary conditions (2.19), (2.20), (2.21) at $y= \pm 1$;

(v) $\vec{v}$ satisfies the boundary conditions (1.14) and (1.15) at $x= \pm N$;

(vi) Near the points $(0, \pm 1) \vec{v}$ has a "sharp Newtonian singularity" namely,

$$
\psi=C r^{\frac{3}{2}}\left(\cos \frac{3 \theta}{2}-\cos \frac{\theta}{2}\right)
$$

where $(r, \theta)$ are polar coordinates about each of the two points with $\theta=0$ in the direction of the solid wall.

In the sequel we always assume that $\overrightarrow{v_{0}} \in V_{0}$. However, condition (vi) can be relaxed to allow a Newtonian singularity of the form established by Richardson for the Newtonian stick-slip problem (cf. [12]); see Remark 12.2.

The main difficulty in solving Problem $(\mathbf{L})$ is inverting the hyperbolic operator $K$ (with $\vec{v}_{0}$ in $V_{0}$ ) defined in (2.4) with boundary condition (2.7).

The solution of Problem (L) will be divided into several steps. The first step is solving the following problem:

Definition 2.3 Problem $\left(\mathbf{H}_{0}\right)$. Let $K$ be the hyperbolic operator defined in (2.4). Given any $F$ (from a class of functions), find $T$ (in a corresponding class of functions to be defined later on) such that

$$
K T=F \text { in } \Sigma \text {, and } T \text { satisfies (2.7). }
$$

Our strategy for solving Problem (L), is as follows:

Given a velocity field $\overrightarrow{\vec{v}}$ in a class $\mathcal{A}$, to be defined later on, we set

$$
F=\epsilon\left(D_{0}+\widetilde{D}\right)
$$

where $D_{0}$ is the vector $D$ corresponding to $\overrightarrow{v_{0}}$ and $\widetilde{D}$ is the vector $D$ corresponding to $\overrightarrow{\vec{v}}$. In Part 2 we solve Problem $\left(\mathbf{H}_{0}\right)$ for any such $\overrightarrow{\vec{v}}$, and in Part 3 we solve the elliptic problem (2.18)-(2.20), (2.21) and (1.14), (1.15) corresponding to $\vec{v}=\overrightarrow{\widetilde{v}}$ and $T=K^{-1}\left(\epsilon\left(D_{0}+\widetilde{D}\right)\right)$. Denoting this solution by $\psi^{*}$ we define the velocity field $\overrightarrow{v^{*}}$ by

$$
\overrightarrow{v^{*}}=\left(\psi_{y}^{*},-\psi_{x}^{*}\right)
$$

and write $\overrightarrow{v^{*}}=W[\overrightarrow{\vec{v}}]$.

In Part 4 we shall prove that $W$ maps $\mathcal{A}$ into itself and is a contraction. Hence $W$ has a unique fixed point in $\mathcal{A}$, and this determines the unique solution of Problem $(L)$. 


\section{$3 \quad$ An auxiliary linear problem}

Consider Problem $\left(\mathbf{H}_{0}\right)$ with

$$
F=\epsilon\left(D_{0}+\widetilde{D}\right) \equiv \epsilon D
$$

If we write $T=U V$ where $U$ is a $3 \times 3$ matrix and $V$ is a column vector with three components, then (recalling(2.4)), equation (2.5) becomes

$$
U V+\alpha_{1}\left(\frac{d U}{d s} V+U \frac{d V}{d s}+A\left(\overrightarrow{v_{0}}\right) U V\right)=\epsilon D
$$

where (by Definition 2.2 (iii))

$$
\frac{d}{d s}=\overrightarrow{v_{0}} \cdot \nabla
$$

We shall require that $U=U(s)$ be a solution of

$$
\frac{d U}{d s}+A\left(\overrightarrow{v_{0}}\right) U=0
$$

where $s$ is the parameter along a characteristic curve; $U$ depends of course also on the initial point of the characteristic curve. Then $V(s)$, the value of $V$ at the point corresponding to the parameter $s$ is related to $V\left(s_{0}\right)$ (with $s_{0}<s$ ) by the formula

$$
V(s)=e^{\frac{-s+s_{0}}{\alpha_{1}}} V\left(s_{0}\right)+\epsilon \alpha_{1}^{-1} \int_{s_{0}}^{s} e^{\frac{-s+\sigma}{\alpha_{1}}} U^{-1}(\sigma) D(x(\sigma), y(\sigma)) d \sigma .
$$

In this section we solve the linear problem (3.1) and then use it in Part 2 to solve Problem $\left(\mathbf{H}_{0}\right)$. By a classical result

$$
\frac{d}{d s} \operatorname{det} U+\left(\operatorname{Tr} A\left(\overrightarrow{v_{0}}\right)\right) \operatorname{det} U=0,
$$

and, since $\operatorname{Tr} A\left(\overrightarrow{v_{0}}\right)=0$,

$$
\operatorname{det} U\left(s_{1}\right)=\operatorname{det} U\left(s_{0}\right) \text { for any } s_{1}, s_{0}
$$

which implies that the matrix $U\left(s_{1}\right)$ is invertible provided we choose an initial matrix $U\left(s_{0}\right)$ which is invertible.

We shall analyze the matrix $U$ in different regions of $\Sigma$. First we consider it in a neighborhood of the point $(0,1)$ or $(0,-1)$ and use polar coordinates for which the fluid lies in $0<\theta<\pi, \theta=0$ is the solid wall and $\theta=\pi$ is the linearized free boundary (see Figure 1). Since $\overrightarrow{v_{0}} \in V_{0}$, it follows that the characteristics are solutions of

$$
\begin{aligned}
r \frac{d \theta}{d s} & =-\psi_{r}=-\frac{3}{2} r^{\frac{1}{2}} f(\theta), \\
\frac{d r}{d s} & =\frac{1}{r} \psi_{\theta}=r^{\frac{1}{2}} f^{\prime}(\theta)
\end{aligned}
$$


where

$$
f(\theta)=\cos \frac{3 \theta}{2}-\cos \frac{\theta}{2}
$$

(we have taken, for simplicity, $C=1$ in (2.22)).

This system can be solved for any initial $\left.r\right|_{s=s_{0}}=r_{0},\left.\theta\right|_{s=s_{0}}=\theta_{0}$. We shall denote the solution by $r\left(s ; r_{0}, \theta_{0}\right), \theta\left(s ; r_{0}, \theta_{0}\right)$. Along characteristics

$$
\frac{d}{d s}=\frac{d \theta}{d s} \frac{d}{d \theta}=-\frac{3}{2} r^{-\frac{1}{2}} f(\theta) \frac{d}{d \theta} .
$$

The matrix $A\left(\overrightarrow{v_{0}}\right)$ takes the very simple form

$$
A\left(\overrightarrow{v_{0}}\right)=r^{-\frac{1}{2}} G(\theta)
$$

where for general $d$ (i.e., when (1.22) is not imposed)

$$
G(\theta)=\left(\begin{array}{ccc}
(2 a-c-2 d) \theta & (-c+2 d) \theta & -2-2(d-a) \\
(c-2 d) \theta & (-2 a+c+2 d) \theta & 2-2(d-a) \\
(1-a+c) & (-1-a+c) & 0
\end{array}\right)+O\left(\theta^{2}\right)
$$

near $\theta=0$ and

$$
\begin{aligned}
G(\theta)= & \left(\begin{array}{ccc}
c-2 a+2 d & c-2 d & \frac{11}{2}(\pi-\theta) \\
-c+2 a & 2 a-c-2 d & -\frac{11}{2}(\pi-\theta) \\
-\frac{11}{4}(\pi-\theta) & \frac{11}{4}(\pi-\theta) & 0
\end{array}\right) \\
& +O(\pi-\theta)
\end{aligned}
$$

near $\theta=\pi$.

Thus (3.1) can be written in the form

$$
-\frac{3}{2} f(\theta) \frac{d U}{d \theta}+G(\theta) U=0
$$

or,

$$
\frac{d U}{d \varphi}+\widetilde{G}(\varphi) U=0
$$

where

$$
\varphi=-\frac{2}{3} \int_{1}^{\theta} \frac{d \vartheta}{f(\vartheta)}
$$

and $\widetilde{G}(\varphi(\theta))=G(\theta)$. Notice that $f(\theta)<0$ for $0<\theta<\pi$ and $f(\theta)=-\theta^{2}+$ $O\left(\theta^{4}\right)$ as $\theta \rightarrow 0, f(\theta)=-2(\pi-\theta)+O\left((\pi-\theta)^{3}\right)$ as $\theta \rightarrow \pi$. Hence as $\theta$ increases from $\theta=0$ to $\theta=\pi, \varphi$ increases from $\varphi=-\infty$ to $\varphi=\infty$, and

$$
\begin{aligned}
\varphi & \sim-\frac{2}{3} \frac{1}{\theta}\left(1+O\left(\theta^{2}\right)\right) \quad \text { as } \theta \rightarrow 0, \\
\varphi & \sim-\frac{4}{3} \log (\pi-\theta)\left(1+O\left((\pi-\theta)^{2}\right)\right) \quad \text { as } \theta \rightarrow \pi .
\end{aligned}
$$

Consider first the case $\theta \sim 0$. The eigenvalues of $G(\theta)$ are

$$
\begin{aligned}
\lambda_{0} & =-2 \frac{3 d a-2 a^{2}-3 d c+2 a c}{1-d a+d c+a^{2}-a c} \theta+o\left(\theta^{2}\right) \equiv-2 b \theta+o\left(\theta^{2}\right) \\
\lambda_{ \pm} & = \pm 2 i \sqrt{1-a c-d a+d c+a^{2}}+\frac{3 d a-2 a^{2}-3 d c+2 a c}{1-d a+d c+a^{2}-a c} \theta+o\left(\theta^{2}\right) \\
& \equiv \pm 2 \gamma i+b \theta+o\left(\theta^{2}\right) .
\end{aligned}
$$


One can verify that

$$
G(\theta)=M_{-}^{-1}(\theta) \Lambda(\theta) M_{-}(\theta)
$$

where

$$
\Lambda(\theta)=\left(\begin{array}{ccc}
\lambda_{0} & 0 & 0 \\
0 & \lambda_{+} & 0 \\
0 & 0 & \lambda_{-}
\end{array}\right)
$$

and $M_{-}(\theta)$ is smooth in $\theta$. The precise expression for $M_{-}(0)$ is complicated; however, in case $d=a$, it simplifies to

$$
M_{-}(0)=\left(\begin{array}{ccc}
\frac{1}{2}(1-a+c) & \frac{1}{2}(1-a+c) & 0 \\
\frac{1}{4}(-1+a-c) & \frac{1}{4}(1+a-c) & \frac{1}{2} i \\
\frac{1}{4}(-1+a-c) & \frac{1}{4}(1+a-c) & -\frac{1}{2} i
\end{array}\right)
$$

and then

$$
M_{-}^{-1}(0)=\left(\begin{array}{ccc}
\frac{1+a-c}{1-a+c} & -1 & -1 \\
1 & 1 & 1 \\
0 & -i & i
\end{array}\right)
$$

Equation (3.8) can be written as

$$
-\frac{3}{2} f(\theta) M_{-}(\theta) \frac{d U}{d \theta}+\Lambda(\theta) M_{-}(\theta) U=0
$$

or,

$$
-\frac{3}{2} f(\theta) \frac{d\left(M_{-}(\theta) U\right)}{d \theta}+\Lambda(\theta)\left(M_{-}(\theta) U\right)=-\frac{3}{2} f(\theta) \frac{d M_{-}(\theta)}{d \theta} U
$$

The homogeneous version of equation (3.13) (i.e. the right hand side is zero) has a general solution of the form

$$
\begin{aligned}
& e^{\frac{2}{3} \int^{\theta} \frac{\Lambda(\eta)}{f(\eta)} d \eta} M_{-}(0) C_{-} \\
& =\left(\begin{array}{ccc}
\theta^{\frac{4}{3} b}(1+O(\theta)) & 0 & 0 \\
0 & \theta^{-\frac{2}{3} b} e^{\frac{4}{3} \frac{1}{\theta} \gamma i}(1+O(\theta)) & 0 \\
0 & 0 & \theta^{-\frac{2}{3} b} e^{-\frac{4}{3} \frac{1}{\theta} \gamma i}(1+O(\theta))
\end{array}\right) C_{-}
\end{aligned}
$$

and therefore the general solution of equation (3.13) is,

$$
M_{-}(\theta) U(\theta)=e^{\frac{2}{3} \int^{\theta} \frac{\Lambda(\eta)}{f(\eta)} d \eta} M_{-}(0) C_{-}+\frac{3}{2} \int_{0}^{\theta} e^{\frac{2}{3} \int_{\theta^{\prime}}^{\theta} \frac{\Lambda(\eta)}{f(\eta)} d \eta} f\left(\theta^{\prime}\right) \frac{d M_{-}\left(\theta^{\prime}\right)}{d \theta} U\left(\theta^{\prime}\right) d \theta^{\prime}
$$

We can easily estimate

$$
\left|e^{\frac{2}{3} \int_{\theta^{\prime}}^{\theta} \frac{\Lambda(\eta)}{f(\eta)} d \eta}\right| \leq C\left(\frac{\theta}{\theta^{\prime}}\right)^{\frac{4}{3} b}
$$

If we assume that

$$
|U(\theta)| \leq \theta^{-\frac{2}{3} b}
$$


then we get (since $b<\frac{3}{2}$ by $(1.23)$ ),

$$
\left|\frac{3}{2} \int_{0}^{\theta} e^{\frac{2}{3} \int_{\theta^{\prime}}^{\theta} \frac{\Lambda(\eta)}{f(\eta)} d \eta} f\left(\theta^{\prime}\right) \frac{d M_{-}\left(\theta^{\prime}\right)}{d \theta^{\prime}} U\left(\theta^{\prime}\right) d \theta^{\prime}\right| \leq C \theta^{\frac{4}{3} b} \theta^{3-2 b} .
$$

This yields

$$
\begin{aligned}
& M_{-}(\theta) U(\theta) \\
= & \left(\begin{array}{ccc}
\theta^{\frac{4}{3} b}(1+O(\theta)) & 0 & 0 \\
0 & \theta^{-\frac{2}{3} b} e^{\frac{4}{3} \frac{1}{\theta} \gamma i}(1+O(\theta)) & 0 \\
0 & 0 & \theta^{-\frac{2}{3} b} e^{-\frac{4}{3} \frac{1}{\theta} \gamma i}(1+O(\theta))
\end{array}\right) M_{-}(0) C_{-} \\
& +\theta^{\frac{4}{3} b} O\left(\theta^{3-2 b}\right)
\end{aligned}
$$

The second term on the right hand side of (3.14) is small with respect to each term in the matrix since $b<\frac{3}{2}$. A straightforward fixed point argument can now be used to establish the following lemma:

Lemma 3.1 For any nonsingular matrix $C_{-}$with real elements there exists a unique nontrivial solution of (3.8) near $\theta=0$ of the form

$$
\begin{gathered}
U=M_{-}^{-1}(0) \\
\times\left(\begin{array}{ccc}
\theta^{\frac{4}{3} b}(1+O(\theta)) & 0 & 0 \\
0 & \theta^{-\frac{2}{3} b} e^{\frac{4}{3} \theta^{-1} \gamma i}(1+O(\theta)) & 0 \\
0 & 0 & \theta^{-\frac{2}{3} b} e^{-\frac{4}{3} \theta^{-1} \gamma i}(1+O(\theta))
\end{array}\right) M_{-}(0) C_{-} \\
+O\left(\theta^{\frac{4}{3} b+b_{0}}\right) \\
\text { as } \theta \rightarrow 0
\end{gathered}
$$

where $M_{-}(0)$ is given by (3.12) and $b_{0}=\frac{3}{2}-b$.

Next we consider the behavior of $U(\theta)$ near $\theta=\pi$, or as $\varphi \rightarrow \infty$ (see (3.11)). In this case

$$
\widetilde{G}(\varphi) \sim\left(\begin{array}{ccc}
-2 a+c+2 d & c-2 d & 0 \\
-c+2 d & 2 a-c-2 d & 0 \\
0 & 0 & 0
\end{array}\right)+O\left(e^{-\frac{3}{4} \varphi}\right) .
$$

The eigenvalues of $\widetilde{G}(\varphi)$ are

$$
\begin{aligned}
& \lambda_{0}=O\left(e^{-\frac{3}{4} \varphi}\right) \\
& \lambda_{ \pm}= \pm 2 \sqrt{b^{\prime}} i \pm O\left(e^{-\frac{3}{4} \varphi}\right)
\end{aligned}
$$

where $b^{\prime}$ is defined in (1.19); in case $d=a$, we have $b^{\prime}=4 b$. One can verify, when $d=a$, that

$$
\widetilde{G}(\varphi)=M_{+}^{-1}(\theta) \Lambda_{0}^{\prime}(\theta) M_{+}(\theta)=\left(\begin{array}{ccc}
c & c-2 a & 0 \\
-c+2 a & -c & 0 \\
0 & 0 & 0
\end{array}\right)+O\left(e^{-\frac{3}{4} \varphi}\right)
$$


where

$$
\begin{gathered}
\Lambda_{0}^{\prime}(\pi)=\left(\begin{array}{ccc}
0 & 0 & 0 \\
0 & -2 \sqrt{b^{\prime}} i & 0 \\
0 & 0 & 2 \sqrt{b^{\prime}} i
\end{array}\right), \\
M_{+}(\pi)=\left(\begin{array}{ccc}
0 & 0 & 1 \\
\frac{1}{4} \frac{i}{\sqrt{b^{\prime}}}(-c+2 a) & -\frac{1}{4} i \frac{2 i \sqrt{b^{\prime}}+c}{\sqrt{b^{\prime}}} & 0 \\
-\frac{1}{4} \frac{i}{\sqrt{b^{\prime}}}(-c+2 a) & \frac{1}{4} i \frac{-2 i \sqrt{b^{\prime}}+c}{\sqrt{b^{\prime}}} & 0
\end{array}\right),
\end{gathered}
$$

and

$$
M_{+}^{-1}(\pi)=\left(\begin{array}{ccc}
0 & \frac{-2 \sqrt{b^{\prime}} i+c}{-c+2 a} & \frac{2 \sqrt{b^{\prime}} i+c}{-c+2 a} \\
0 & 1 & 1 \\
1 & 0 & 0
\end{array}\right) .
$$

Proceeding as above we obtain the following analog of Lemma 3.1.

Lemma 3.2 For any nonsingular matrix $C_{+}$with real elements, there exists a unique nontrivial solution of (3.8) near $\theta=\pi$ of form

$$
\begin{array}{r}
U=M_{+}^{-1}(\pi)\left(\begin{array}{ccc}
1 & 0 & 0 \\
0 & e^{-\frac{8}{3} \sqrt{b^{\prime}} \log (\pi-\theta) i} & 0 \\
0 & 0 & e^{\frac{8}{3} \sqrt{b^{\prime}} \log (\pi-\theta) i}
\end{array}\right) M_{+}(\pi) C_{+} \\
\\
\quad+O\left((\pi-\theta)^{\beta}\right) \quad \text { as } \theta \rightarrow \pi
\end{array}
$$

where $\beta$ is a small positive number.

We next consider the matrix $U$ away from the points $(0, \pm 1)$, in a neighborhood of $\left\{y= \pm 1,-N<x<-\delta_{0}<0\right\}$ where the non-slip boundary conditions hold. From the boundary conditions on $\overrightarrow{v_{0}}=\left(v_{0, x}, v_{0, y}\right)$ and (iii) of Definition 2.2 , we get

$$
\begin{aligned}
v_{0, x} & \sim a_{1}(x)(|y|-1) \text { as }|y| \rightarrow 1, \quad-N<x<0, a_{1}(x) \neq 0, \\
v_{0, y} & \sim \frac{a_{1}^{\prime}(x)}{2}(|y|-1)^{2} \text { as }|y| \rightarrow 1, \quad-N<x<0, \\
\frac{\partial v_{0, x}}{\partial x} & \sim a_{1}^{\prime}(x)(|y|-1) \text { as }|y| \rightarrow 1, \quad-N<x<0, \\
\frac{\partial v_{0, x}}{\partial y} & \sim a_{1}(x) \text { as }|y| \rightarrow 1, \quad-N<x<0, \\
\frac{\partial v_{0, y}}{\partial x} & \sim \frac{a_{1}^{\prime \prime}(x)}{2}(|y|-1)^{2} \text { as }|y| \rightarrow 1, \quad-N<x<0 .
\end{aligned}
$$

Similarly, in the neighborhood of $\left\{y= \pm 1,0<\delta_{0}<x<N\right\}$,

$$
\begin{aligned}
v_{0, x} & \sim b_{1}(x) \text { as }|y| \rightarrow 1,0<x<N, \\
v_{0, y} & \sim b_{1}^{\prime}(x)(|y|-1) \text { as }|y| \rightarrow 1,0<x<N, \\
\frac{\partial v_{0, x}}{\partial x} & \sim b_{1}^{\prime}(x) \text { as }|y| \rightarrow 1,0<x<N, \\
\frac{\partial v_{0, x}}{\partial y} & \sim-b_{1}^{\prime \prime}(x)(|y|-1)^{2} \text { as }|y| \rightarrow 1,0<x \leq N, \\
\frac{\partial v_{0, y}}{\partial x} & \sim b_{1}^{\prime \prime}(x)(|y|-1) \text { as }|y| \rightarrow 1,0<x \leq N .
\end{aligned}
$$


By a straightforward calculation

$$
\begin{aligned}
A & =A\left(\overrightarrow{v_{0}}\right)=\left(\begin{array}{ccc}
0 & 0 & 1 \\
0 & 0 & -1 \\
-\frac{1+c-a}{2} & \frac{1-c+a}{2} & 0
\end{array}\right) a_{1}(x)+O(1-|y|) \\
& =-\frac{1}{2} G(0) a_{1}(x)+O(1-|y|) \text { if }-N \leq x<-\delta_{0} \text { and }|y| \rightarrow 1,
\end{aligned}
$$

and

$$
A=\left(\begin{array}{ccc}
c & c-2 a & 0 \\
-c+2 a & -c & 0 \\
0 & 0 & 0
\end{array}\right) b_{1}^{\prime}(x)+O(1-|y|) \quad \text { if } \delta_{0}<x<N \quad \text { and }|y| \rightarrow 1
$$

where $G_{0}$ is the same matrix as in (3.6) with $\theta=0$.

Along characteristics passing near $|y|=1, x<0$

$$
d s=(1-|y|)^{-1} a_{1}^{-1}(x)(1+O(1-|y|)) d x .
$$

Proceeding as in the proof of Lemma 3.1 we get

$$
\frac{d\left(M_{-} U\right)}{d s}-\frac{1}{2} \Lambda_{0} a_{1}(x)\left(M_{-} U\right)=O(1-|y|) U
$$

and, by integration,

$$
\begin{aligned}
& U=M_{-}^{-1}(x) \\
& \times\left(\begin{array}{ccc}
\eta^{\frac{4}{3} b}(1+o(\eta)) & 0 & 0 \\
0 & \eta^{-\frac{2}{3} b} e^{-\frac{g(x)}{\eta} \gamma i}(1+o(\eta)) & 0 \\
0 & 0 & \eta^{-\frac{2}{3} b} e^{\frac{g(x)}{\eta} \gamma i}(1+o(\eta)
\end{array}\right) M_{-}(x) C_{-} \\
& +\eta^{-\frac{2}{3} b} O\left(\eta^{b_{0}}\right) \quad \text { for }-N \leq x<0
\end{aligned}
$$

where $\eta=1-|y|, g(x)$ is a bounded and regular function, and $C_{-}$is any nonsingular matrix with real elements. Notice that the structure given by (3.17) implies very strong oscillations as $y \rightarrow \pm 1$.

The situation for $\delta_{0}<x \leq N$ is much simpler. We have the equation

$b_{1}(x)(|y|-1) \frac{\partial U}{\partial y}+b_{1}(x) \frac{\partial U}{\partial x}+\left(\begin{array}{ccc}c & c-2 a & 0 \\ -c+2 a & -c & 0 \\ 0 & 0 & 0\end{array}\right) b_{1}^{\prime}(x) U=O(1-|y|) U$

whose solution can be written in the form

$$
U=M_{+}^{-1}(x)\left(\begin{array}{ccc}
1 & 0 & 0 \\
0 & e^{\frac{8}{3} \sqrt{b} \log (1-y) i} & 0 \\
0 & 0 & e^{-\frac{8}{3} \sqrt{b} \log (1-y) i}
\end{array}\right) M_{+}(x) C_{+}+O(1-|y|)
$$

where $M_{+}(x)$ a matrix with real bounded elements.

The assumptions on $\overrightarrow{v_{0}}$ ensure that equation (3.1) has a unique solution through any point in $\Sigma$; the solution can be extended from $x=-N$ to $x=+N$, and it is in $C^{1+\alpha}$ in any region $|y| \leq 1-\varepsilon_{0}\left(\varepsilon_{0}>0\right)$; near $y=1$ the solution behaves in accordance with Lemmas 3.1, 3.2 and (3.17), (3.18). The following theorem summarizes the results of this section. 
Theorem 3.3 Given initial condition $U_{0}(y)$ at $x=-N$, with $\operatorname{det} U_{0}(y) \neq 0$ for $|y| \leq 1$, Equation (3.1) has a unique solution along any characteristic in $\Sigma\left(\right.$ from $x=-N$ to $x=N$ ) with $\left.U\right|_{x=-N}=U_{0}(y)$, and $\operatorname{det} U \neq 0$ for all $s$; $U(x, y)$ satisfies the following properties in $\Sigma$ :

1) In the disc of radius $\delta$ around the points $(0, \pm 1)$ intersected with $\Sigma$,

$$
U=U(\theta)
$$

with

$$
\begin{gathered}
U(\theta)=M_{-}^{-1}(0) \\
\times\left(\begin{array}{ccc}
\theta^{\frac{4}{3} b}(1+O(\theta)) & 0 & 0 \\
0 & \theta^{-\frac{2}{3} b} e^{\frac{4}{3} \theta^{-1} \gamma i}(1+O(\theta)) & 0 \\
0 & \theta^{-\frac{2}{3} b} e^{-\frac{4}{3} \theta^{-1} \gamma i}(1+O(\theta))
\end{array}\right) \\
+O\left(\theta^{-\frac{2}{3} b+b_{0}}\right) \\
U(\theta)=M_{+}^{-1}(\pi)\left(\begin{array}{ccc}
e^{-\frac{8}{3} \sqrt{b^{\prime}} \log (\pi-\theta) i} & 0 \\
0 & e^{\frac{8}{3} \sqrt{b^{\prime}} \log (\pi-\theta) i} & 0 \\
0 & 0 & 1
\end{array}\right) M_{+}(\pi) C_{+} \\
+O\left((\pi-\theta)^{\beta}\right) \\
\text { as } \theta \rightarrow \pi
\end{gathered}
$$

for some $\beta>0$ and for some real constant matrices $C_{+}$and $C_{-}$whose elements are $O(1)$;

2) In the neighborhood of the lines $y= \pm 1$ outside the disc of radius $\delta$ around the point $(0, \pm 1)$ :

$$
\begin{aligned}
& U(x, y)=M_{-}^{-1}(x) \\
& \times\left(\begin{array}{ccc}
\eta^{\frac{4}{3} b}(1+o(\eta)) & 0 & 0 \\
0 & \eta^{-\frac{2}{3} b} e^{\frac{4}{3} \frac{g(x)}{\eta} \gamma i}(1+o(\eta)) & 0 \\
0 & 0 & \eta^{-\frac{2}{3} b} e^{-\frac{4}{3} \frac{g(x)}{\eta} \gamma i}(1+o(\eta))
\end{array}\right) M_{-}(x) C_{-} \\
& +O\left(\eta^{-\frac{2}{3} b+b_{0}}\right) \text { as as }|y| \rightarrow 1, \text { for }-N<x<0 \text { as }|y| \rightarrow 1
\end{aligned}
$$

where

$$
\eta=1-|y|
$$

and

$$
\begin{gathered}
U(x, y)=M_{+}^{-1}(x)\left(\begin{array}{ccc}
e^{-\frac{8}{3} \sqrt{b^{\prime}}(\log \eta) i} & 0 & 0 \\
0 & e^{\frac{8}{3} \sqrt{b^{\prime}}(\log \eta) i} & 0 \\
0 & 0 & 1
\end{array}\right) M_{+}(x) C_{+} \\
+O(\eta) \text { as }|y| \rightarrow 1, \text { for } 0<x<N
\end{gathered}
$$

for some matrices $M_{-}(x), M_{+}(x)$ and $C_{-}, C_{+}$with bounded real elements;

3) In the remaining portion of the strip $\Sigma, U$ is in $C^{\alpha}$. 
Remark 3.1. From the proof of Theorem 3.3 one can check that the error terms $O\left(\theta^{-\frac{2}{3} b+b_{0}}\right)$ depend in a $C^{\alpha}$ way on $\theta$.

\section{Part II}

\section{Analysis of the hyperbolic problem}

In this part we analyze the solution of the hyperbolic Problem $\left(\mathbf{H}_{0}\right)$ and derive $C^{\alpha}$ estimates. We deduce all the estimates for the case $d=a$.

The most delicate analysis occurs in the neighborhood of the points $( \pm 1,0)$. This analysis is presented in this and the next three sections. In Section 8 we complete the derivation of the $C^{\alpha}$ bounds in the entire flow region.

\section{$4 \quad L^{\infty}$ bound on the principal term}

We shall continue to adopt the notation of Section 3 with polar coordinates $(r, \theta)$ centered at $( \pm 1,0)$ such that $\theta=0$ is in the direction of the fixed boundary and the fluid lies in the region $0<\theta<\pi$. Consider an arbitrary point in this neighborhood and denote its polar coordinates by $\left(r_{f}, \theta_{f}\right)$. We shall parametrize the characteristic line through $\left(r_{f}, \theta_{f}\right)$ by $\theta$ instead of the parameter $s$. The relation between $\theta$ and $s$ is given by (3.3) and (3.4), i.e.,

$$
r \frac{d \theta}{d s}=v_{\theta}=-\psi_{r}=-\frac{3}{2} r^{\frac{1}{2}} f(\theta), f(\theta) \text { as in }(3.4)
$$

together with the fact that $\psi$ is constant along the characteristic, that is (by $(2.22))$

$$
r^{\frac{3}{2}}(\theta) f(\theta)=r_{0}^{\frac{3}{2}} f\left(\theta_{0}\right)
$$

where $\left(r_{0}, \theta_{0}\right)$ is some "reference point" that we will take such that $r_{0}=\delta_{0}$, $0<\theta_{0}<\widetilde{\theta_{0}}$ where $\delta_{0}, \widetilde{\theta_{0}}$ are small and fixed, and $\widetilde{\theta_{0}}<<\delta_{0}$.

Using (3.2), (4.1), (4.2), we can write the solution of the hyperbolic problem in the form

$$
T=T_{h}+T_{s}
$$

where

$$
\begin{gathered}
T_{h}\left(\theta_{f}\right)=U\left(\theta_{f}\right) U^{-1}\left(\theta_{0}\right) e^{-\frac{2}{3 \alpha_{1}} \int_{\theta f}^{\theta} \frac{r(\theta)^{\frac{1}{2}}}{|f(\theta)|} d \theta} T\left(\theta_{0}\right) \\
T_{s}\left(\theta_{f}\right)=-\frac{2 \epsilon}{3 \alpha_{1}} U\left(\theta_{f}\right) \int_{\theta_{0}}^{\theta_{f}} U^{-1}(\theta) e^{-\frac{2}{3 \alpha_{1}} \int_{\theta f}^{\theta} \frac{r(\theta)^{\frac{1}{2}}}{f(\theta) \mid} d \theta}\left(r^{\frac{1}{2}} D\right) \frac{d \theta}{f(\theta)} .
\end{gathered}
$$

We first consider $T_{s}\left(\theta_{f}\right)$ and decompose it into two terms

$$
\begin{gathered}
T_{s}\left(\theta_{f}\right)=-\frac{2 \epsilon}{3 \alpha_{1}} \int_{\theta_{0}}^{\theta_{f}} U\left(\theta_{f}\right) U^{-1}(\theta) e^{-\frac{2}{3 \alpha_{1}} \int_{\theta f}^{\theta} \frac{r(\theta)^{\frac{1}{2}}}{|f(\theta)|} d \theta}\left(r^{\frac{1}{2}} D_{0}\right) \frac{d \theta}{f(\theta)} \\
-\frac{2 \epsilon}{3 \alpha_{1}} \int_{\theta_{0}}^{\theta_{f}} U\left(\theta_{f}\right) U^{-1}(\theta) e^{-\frac{2}{3 \alpha_{1}} \int_{\theta f}^{\theta} \frac{r(\theta)^{\frac{1}{2}}}{\mid f(\theta)} d \theta}\left(r^{\frac{1}{2}} \widetilde{D}\right) \frac{d \theta}{f(\theta)}
\end{gathered}
$$




$$
\equiv I\left(\theta_{0}, \theta_{f}\right)+J\left(\theta_{0}, \theta_{f}\right) .
$$

$I\left(\theta_{0}, \theta_{f}\right)$ is the principal (and most singular) term and $J\left(\theta_{0}, \theta_{f}\right)$ is the (less singular but also less explicit) perturbation. In this section we derive $L^{\infty}$ bounds on $I$ and in Section 5 we obtain $C^{\alpha}$ estimates on $I$. Similar results will be established for $J$ in Sections 6 , 7. Section 8 summarizes, in a convenient form, all these estimates for $T_{s}$ as well as the corresponding estimates for $T_{h}$.

Recall that $\frac{d \theta}{d s}>0$ along characteristics, so that $\theta_{0}<\theta_{f}<\pi$. Let us consider separately the three cases

$$
\theta_{0}<\theta_{f}<\theta_{1}, \theta_{1}<\theta_{f}<\theta_{2}, \theta_{2}<\theta_{f}<\pi
$$

where $\theta_{1}, \theta_{2}$ are chosen so that

$$
\theta_{0}<<\theta_{1}<<1, \quad \pi-\theta_{2}<<1
$$

Lemma 4.1 If $\theta_{0}<\theta_{f}<\theta_{1}$ then $I\left(\theta_{0}, \theta_{f}\right)$ is bounded, i.e.

$$
\left|I\left(\theta_{0}, \theta_{f}\right)\right| \leq C|\epsilon|
$$

for some constant $C$ independent of $\epsilon$.

Proof. We can write

$$
D_{0}=\left(\begin{array}{c}
D_{0, X X} \\
D_{0, Y Y} \\
D_{0, X Y}
\end{array}\right)=\left(\begin{array}{c}
\Psi_{X Y} \\
-\Psi_{X Y} \\
\Psi_{Y Y}-\Psi_{X X}
\end{array}\right)
$$

where

$$
\begin{gathered}
\Psi=r^{\frac{3}{2}} f(\theta), \quad f(\theta)=\cos \frac{3 \theta}{2}-\cos \frac{\theta}{2}, \\
X=r \cos \theta, Y=r \sin \theta .
\end{gathered}
$$

Since

$$
f(\theta)=-\theta^{2}\left(1+O\left(\theta^{2}\right)\right)
$$

we have

$$
D_{0}=\left(\begin{array}{c}
\frac{Y}{|X|^{\frac{3}{2}}} \\
-\frac{Y}{|X|^{\frac{3}{2}}} \\
-\frac{1}{|X|^{\frac{1}{2}}}
\end{array}\right)\left(1+O\left(\theta^{2}\right)\right)=\frac{1}{r^{\frac{1}{2}}}\left(\begin{array}{c}
\theta \\
-\theta \\
-1
\end{array}\right)\left(1+O\left(\theta^{2}\right)\right)
$$

Using (4.2) we compute,

$$
\begin{aligned}
& \int_{\theta f}^{\theta} \frac{r(\theta)^{\frac{1}{2}}}{|f(\theta)|} d \theta=r_{f}^{\frac{1}{2}}\left|f\left(\theta_{f}\right)\right|^{\frac{1}{3}} \int_{\theta f}^{\theta} \frac{1}{|f(\theta)|^{\frac{4}{3}}} d \theta \\
& =\frac{3}{5 \alpha_{1}} r_{f}^{\frac{1}{2}} \theta_{f}^{\frac{2}{3}}\left(\theta_{f}^{-\frac{5}{3}}-\theta^{-\frac{5}{3}}\right)\left(1+O\left(\theta^{2}\right)\right) .
\end{aligned}
$$


By Lemma 3.1 and (4.9)

$$
\begin{gathered}
I\left(\theta_{0}, \theta_{f}\right)=\frac{2 \epsilon}{3 \alpha_{1}} \int_{\theta_{0}}^{\theta_{f}} M_{-}^{-1}(0)\left(\begin{array}{ccc}
\theta_{f}^{\frac{4}{3} b} & 0 & 0 \\
0 & \theta_{f}^{-\frac{2}{3} b} e^{\frac{4}{3} \theta_{f}^{-1} i} & 0 \\
0 & 0 & \theta_{f}^{-\frac{2}{3} b} e^{-\frac{4}{3} \theta_{f}^{-1} i}
\end{array}\right) \\
\times\left(\begin{array}{ccc}
\theta^{-\frac{4}{3} b} & 0 & 0 \\
0 & \theta^{\frac{2}{3} b} e^{-\frac{4}{3} \theta^{-1} i} & 0 \\
0 & 0 & \theta^{\frac{2}{3} b} e^{-\frac{4}{3} \theta^{-1} i}
\end{array}\right) M_{-}(0)\left(\begin{array}{c}
-\theta \\
\theta \\
1
\end{array}\right) \\
\times e^{-\frac{2}{3 \alpha_{1}} \int_{\theta f}^{\theta} \frac{r(\theta) \frac{1}{2}}{|f(\theta)|} d \theta}\left(1+O\left(\theta^{b_{0}}\right)\right) \frac{d \theta}{f(\theta)}
\end{gathered}
$$

Next, using (4.10), making a change of variables $\theta=\theta_{f} u^{-1}$, and setting

$$
\rho=\frac{2}{5 \alpha_{1}} \frac{r_{f}^{\frac{1}{2}}}{\theta_{f}}
$$

(notice that $\rho$ may take any value in $(0, \infty)$ ), we get

$$
\begin{aligned}
& I\left(\theta_{0}, \theta_{f}\right)= \\
& \frac{2 \epsilon}{3 \alpha_{1} \theta_{f}} \int_{1}^{\frac{\theta_{f}}{\theta_{0}}} e^{-\rho\left(u^{\frac{5}{3}}-1\right)\left(1+O\left(\theta^{2}\right)\right)} M_{-}^{-1}(0)\left(\begin{array}{ccc}
u^{\frac{4}{3} b} & 0 & 0 \\
0 & u^{-\frac{2}{3} b} e^{-\frac{4}{3 \theta_{f}}(u-1) i} & 0 \\
0 & 0 & u^{-\frac{2}{3} b} e^{-\frac{4}{3 \theta_{f}}(u-1) i}
\end{array}\right) \\
& \times M_{-}(0) \frac{\theta_{f}}{u}\left(\begin{array}{c}
-1 \\
1 \\
\frac{u}{\theta_{f}}
\end{array}\right)\left(1+O\left(\theta^{b_{0}}\right)\right) d u .
\end{aligned}
$$

We first consider the case $O\left(\theta^{b_{0}}\right) \equiv 0$.

Introducing the notation

$$
\lambda=\frac{1}{4}(1-a+c), \quad \mu=\frac{1}{4}(1+a-c)
$$

we have

$$
\begin{aligned}
& M_{-}^{-1}(0)\left(\begin{array}{ccc}
u^{\frac{4}{3} b} & 0 & 0 \\
0 & u^{-\frac{2}{3} b} e^{-\frac{4}{3 \theta_{f}}(u-1) i} & 0 \\
0 & 0 & u^{-\frac{2}{3} b} e^{\frac{4}{3 \theta_{f}}(u-1) i}
\end{array}\right) M_{-}(0) \\
& =\left(\begin{array}{ccc}
\frac{u^{-\frac{2}{3} b} \lambda \cos \frac{4}{3 \theta_{f}}(u-1)+\mu u^{\frac{4}{3} b}}{\mu+\lambda} & -\mu \frac{u^{-\frac{2}{3} b} \cos \frac{4}{3 \theta_{f}}(u-1)-u^{\frac{4}{3} b}}{\mu+\lambda} & -\frac{1}{2} u^{-\frac{2}{3} b} \frac{\sin \frac{4}{3 \theta_{f}}(u-1)}{\mu+\lambda} \\
-\lambda \frac{u^{-\frac{2}{3} b} \cos \frac{4}{3 \theta_{f}}(u-1)-u^{\frac{4}{3} b}}{\mu+\lambda} & \frac{u^{-\frac{2}{3} b} \mu \cos \frac{4}{3 \theta_{f}}(u-1)+u^{\frac{4}{3} b} \lambda}{\mu+\lambda} & \frac{1}{2} u^{-\frac{2}{3} b \frac{\sin \frac{4}{3 \theta_{f}}(u-1)}{\mu+\lambda}} \\
2 u^{-\frac{2}{3} b} \lambda \sin \frac{4}{3 \theta_{f}}(u-1) & -2 u^{-\frac{2}{3} b \mu \sin \frac{4}{3 \theta_{f}}(u-1)} & u^{-\frac{2}{3} b} \cos \frac{4}{3 \theta_{f}}(u-1)
\end{array}\right)
\end{aligned}
$$


so that

$$
\begin{aligned}
& \theta_{f}^{-1}\left(\begin{array}{cccc}
\frac{u^{-\frac{2}{3} b} \lambda \cos \frac{4}{3 \theta_{f}}(u-1)+\mu u^{\frac{4}{3} b}}{\mu+\lambda} & -\mu \frac{u^{-\frac{2}{3} b} \cos \frac{4}{3 \theta_{f}}(u-1)-u^{\frac{4}{3} b}}{\mu+\lambda} & -\frac{1}{2} u^{-\frac{2}{3} b} \frac{\sin \frac{4}{3 \theta_{f}}(u-1)}{\mu+\lambda} \\
-\lambda \frac{u^{-\frac{2}{3} b} \cos \frac{4}{3 \theta_{f}}(u-1)-u^{\frac{4}{3} b}}{\mu+\lambda} & \frac{u^{-\frac{2}{3} b} \mu \cos \frac{4}{3 \theta_{f}}(u-1)+u^{\frac{4}{3} b} \lambda}{\mu+\lambda} & \frac{1}{2} u^{-\frac{2}{3} b \frac{\sin \frac{4}{3 \theta_{f}}(u-1)}{\mu+\lambda}} \\
2 u^{-\frac{2}{3} b} \lambda \sin \frac{4}{3 \theta_{f}}(u-1) & -2 u^{-\frac{2}{3} b} \mu \sin \frac{4}{3 \theta_{f}}(u-1) & u^{-\frac{2}{3} b} \cos \frac{4}{3 \theta_{f}}(u-1)
\end{array}\right) \\
& \times\left(\begin{array}{c}
-1 \\
1 \\
\frac{u}{\theta_{f}}
\end{array}\right) \frac{\theta_{f}}{u} \\
& =\left(\begin{array}{c}
-\frac{1}{2} u^{-1-\frac{2}{3} b} \frac{2 \lambda \cos \left(\frac{4}{3 \theta_{f}}(u-1)\right)+2 \mu \cos \left(\frac{4}{3 \theta_{f}}(u-1)\right)+\theta_{f}^{-1} u \sin \left(\frac{4}{3 \theta_{f}}(u-1)\right)}{\mu+\lambda} \\
\frac{1}{2} u^{-1-\frac{2}{3} b} \frac{2 \lambda \cos \left(\frac{4}{3 \theta_{f}}(u-1)\right)+2 \mu \cos \left(\frac{4}{3 \theta_{f}}(u-1)\right)+\theta_{f}^{-1} u \sin \left(\frac{4}{3 \theta_{f}}(u-1)\right)}{u^{\mu+\lambda}} \\
-u^{-1-\frac{2}{3} b} 2 \lambda \sin \left(\frac{4}{3 \theta_{f}}(u-1)\right)+2 \mu \sin \left(\frac{4}{3 \theta_{f}}(u-1)\right)-\theta_{f}^{-1} u \cos \left(\frac{4}{3 \theta_{f}}(u-1)\right)
\end{array}\right)
\end{aligned}
$$

Each component of this last vector is a linear combination with uniformly bounded coefficients of the functions

$$
\theta_{f}^{-1} u^{-\frac{2}{3} b} e^{ \pm \frac{4}{3 \theta_{f}}(u-1) i}, u^{-1-\frac{2}{3} b} e^{ \pm \frac{4}{3 \theta_{f}}(u-1) i} .
$$

Remark 4.1. Notice the following important fact: the terms of the form $u^{\frac{4}{3} b}$ have cancelled out; this is due to the special condition $d=a$ which resulted in the simplified matrix $A\left(\overrightarrow{v_{0}}\right)$ in (2.3).

We conclude that $I\left(\theta_{0}, \theta_{f}\right)$ is a linear combination (with coefficients which are uniformly bounded by a constant times $|\epsilon|$ ) of integrals of the form

$$
\begin{aligned}
J & =\theta_{f}^{-1} \int_{1}^{\frac{\theta_{f}}{\theta_{0}}} e^{-\rho\left(u^{\frac{5}{3}}-1\right)\left(1+O\left(\theta^{2}\right)\right)} u^{-\delta} e^{ \pm \frac{4}{3} \frac{i}{\theta_{f}}(u-1)} d u \quad \text { with } \delta=\frac{2 b}{3} \\
K & =\int_{1}^{\frac{\theta_{f}}{\theta_{0}}} e^{-\rho\left(u^{\frac{5}{3}}-1\right)\left(1+O\left(\theta^{2}\right)\right)} u^{-\delta} e^{ \pm \frac{4}{3} \frac{i}{\theta_{f}}(u-1)} d u \quad \text { with } \delta=\frac{2 b}{3}+1
\end{aligned}
$$

To estimate $J$ we write

$$
\begin{gathered}
J=\theta_{f}^{-1} \int_{1}^{\frac{\theta_{f}}{\theta_{0}}} e^{-\rho\left(u^{\frac{5}{3}}-1\right)} u^{-\delta} e^{ \pm \frac{4}{3} \frac{i}{\theta_{f}}(u-1)} d u \\
+\theta_{f}^{-1} \int_{1}^{\frac{\theta_{f}}{\theta_{0}}} e^{-\rho\left(u^{\frac{5}{3}}-1\right)}\left[e^{-\rho\left(u^{\frac{5}{3}}-1\right) O\left(\theta^{2}\right)}-1\right] u^{-\delta} e^{ \pm \frac{4}{3} \frac{i}{\theta_{f}}(u-1)} d u \\
\equiv L_{1}\left(\rho, \theta_{f}\right)+R\left(\rho, \theta_{f}\right)
\end{gathered}
$$

where

$$
\left|R\left(\rho, \theta_{f}\right)\right| \leq \theta_{f}^{-1} \int_{1}^{\frac{\theta_{f}}{\theta_{0}}} e^{-\rho\left(u^{\frac{5}{3}}-1\right)}\left|e^{-\rho\left(u^{\frac{5}{3}}-1\right) O\left(\theta^{2}\right)}-1\right| u^{-\delta} d u
$$




$$
\begin{gathered}
\leq C r_{f}^{\frac{1}{2}} \int_{1}^{\infty} e^{-\rho\left(u^{\frac{5}{3}}-1\right)}\left|\left(u^{\frac{5}{3}}-1\right) u^{-2}\right| u^{-\delta} d u \leq C r_{f}^{\frac{1}{2}} \int_{1}^{\infty} e^{-\rho\left(u^{\frac{5}{3}}-1\right)} u^{-\delta-\frac{1}{3}} d u \\
\leq C r_{f}^{\frac{1}{2}} \rho^{-\left(\frac{2}{3}-\delta\right) \frac{3}{5}} \leq C .
\end{gathered}
$$

Thus it remains to estimate $L_{1}\left(\rho, \theta_{f}\right)$. By a change of variables $1-u=-\theta_{f} v$ we get

$$
\begin{gathered}
L_{1}\left(\rho, \theta_{f}\right)=\int_{0}^{\frac{\theta_{f}}{\theta_{0}}}\left(1+\theta_{f} v\right)^{-\delta} e^{\frac{4}{3} v i} e^{-\rho\left(\left(1+\theta_{f} v\right)^{\frac{5}{3}}-1\right)} d v \\
=\int_{0}^{\infty}\left(1+\theta_{f} v\right)^{-\delta} e^{\frac{4}{3} v i} e^{-\rho\left(\left(1+\theta_{f} v\right)^{\frac{5}{3}}-1\right)} d v+O(1) \equiv \widetilde{L_{1}}\left(\rho, \theta_{f}\right)+O(1) .
\end{gathered}
$$

We may change the path of integration (in the complex plane) to the line $e^{\frac{3 \pi}{10} i} s$, $0<s<+\infty$, to obtain

$\widetilde{L_{1}}\left(\rho, \theta_{f}\right)=e^{\frac{3 \pi}{10} i} \int_{0}^{\infty}\left(1+\theta_{f} e^{\frac{3 \pi}{10} i} s\right)^{-\delta} e^{\frac{4}{3}\left(\cos \frac{3 \pi}{10}\right) s i} e^{-\frac{4}{3}\left(\sin \frac{3 \pi}{10}\right) s} e^{-\rho\left(\left(1+\theta_{f} e^{\frac{3 \pi}{10} i} s\right)^{\frac{5}{3}}-1\right)} d s$.

It is easy to verify that

$$
\operatorname{Re}\left(\left(1+e^{\frac{3 \pi}{10} i} x\right)^{\frac{5}{3}}-1\right)
$$

is positive for $x>0$ and that

$$
\left|\left(1+\theta_{f} e^{\frac{3 \pi}{10} i} s\right)^{-\delta}\right| \leq C .
$$

Therefore

$$
\begin{aligned}
\left|\widetilde{L_{1}}\left(\rho, \theta_{f}\right)\right| & \leq C \int_{0}^{\infty} e^{-\frac{4}{3}\left(\sin \frac{3 \pi}{10}\right) s}\left|e^{-\rho\left(\left(1+\theta_{f} e^{\frac{3 \pi}{10} i} s\right)^{\frac{5}{3}}-1\right)}\right| d s \\
& \leq \int_{0}^{\infty} e^{-\frac{4}{3}\left(\sin \frac{3 \pi}{10}\right) s} d s=\frac{1}{\frac{4}{3} \sin \frac{3 \pi}{10}} .
\end{aligned}
$$

We have thus proved the boundedness of $J$.

To estimate $K$ we write

$$
\begin{gathered}
|K| \leq \int_{1}^{\frac{\theta_{f}}{\theta_{0}}} e^{-\rho\left(u^{\frac{5}{3}}-1\right)} u^{-\delta} d u+\int_{1}^{\frac{\theta_{f}}{\theta_{0}}} e^{-\rho\left(u^{\frac{5}{3}}-1\right)}\left[e^{-\rho\left(u^{\frac{5}{3}}-1\right) O\left(\theta^{2}\right)}-1\right] u^{-\delta} d u \\
\equiv L_{2}\left(\rho, \theta_{f}\right)+R\left(\rho, \theta_{f}\right) .
\end{gathered}
$$

We estimate $R\left(\rho, \theta_{f}\right)$ as in the case of $J$. As for $L_{2}\left(\rho, \theta_{f}\right)$, we have

$$
\begin{aligned}
L_{2}\left(\rho, \theta_{f}\right) & =\int_{1}^{\frac{\theta_{f}}{\theta_{0}}} e^{-\rho\left(u^{\frac{5}{3}}-1\right)} u^{-\delta} d u=\int_{1}^{\infty} e^{-\rho\left(u^{\frac{5}{3}}-1\right)} u^{-\delta} d u+O(1) \\
& \equiv \widetilde{L_{2}}\left(\rho, \theta_{f}\right)+O(1) \text { as } \theta_{f} \rightarrow 0
\end{aligned}
$$

and

$$
\widetilde{L_{2}}\left(\rho, \theta_{f}\right)=\int_{1}^{\infty} e^{-\rho\left(u^{\frac{5}{3}}-1\right)} u^{-\delta} d u=\frac{3}{5} \int_{1}^{\infty} e^{-\rho(v-1)} v^{\frac{3}{5}(1-\delta)-1} d v
$$

which is bounded provided $\delta>1$. This completes the proof of the boundedness of $K$ and thus also of $I\left(\theta_{0}, \theta_{f}\right)$ if $O\left(\theta^{b_{0}}\right) \equiv 0$. Finally, the integral corresponding to $O\left(\theta^{b_{0}}\right)$ in $I\left(\theta_{0}, \theta_{f}\right)$ can be treated by the same argument as the error term in (4.16). 
Lemma 4.2 If $\theta_{1}<\theta_{f}<\theta_{2}$ then $I\left(\theta_{0}, \theta_{f}\right)$ is bounded, i.e.

$$
\left|I\left(\theta_{0}, \theta_{f}\right)\right| \leq C|\epsilon|
$$

for some constant $C$ independent of $\epsilon$.

Proof. We can write

$$
I\left(\theta_{0}, \theta_{f}\right)=\frac{2 \epsilon}{3 \alpha_{1}} U\left(\theta_{f}\right)\left(\int_{\theta_{0}}^{\theta_{1}}+\int_{\theta_{1}}^{\theta_{f}}\right) \equiv I_{2}+I_{3}
$$

where

$$
\begin{aligned}
I_{2} & =\frac{2 \epsilon}{3 \alpha_{1}} \int_{\theta_{0}}^{\theta_{1}} U\left(\theta_{f}\right) U^{-1}(\theta) e^{-\frac{2}{3 \alpha_{1}} \int_{\theta f}^{\theta_{1}} \frac{r(\eta)}{\mid f(\eta)} d \eta} e^{-\frac{2}{3 \alpha_{1}} \int_{\theta 1}^{\theta} \frac{r(\eta)^{\frac{1}{2}}}{f(\eta) \mid} d \eta}\left(r^{\frac{1}{2}} D_{0}\right) \frac{d \theta}{f(\theta)}= \\
& =\frac{2 \epsilon}{3 \alpha_{1}} e^{-\frac{2}{3 \alpha_{1}} \int_{\theta f}^{\theta_{1}} \frac{r(\eta)^{\frac{1}{2}}}{f(\eta) \mid} d \eta} \int_{\theta_{0}}^{\theta_{1}} U\left(\theta_{f}\right) U^{-1}(\theta) e^{-\frac{2}{3 \alpha_{1}} \int_{\theta 1}^{\theta} \frac{r(\eta)^{\frac{1}{2}}}{f(\eta) \mid} d \eta}\left(r^{\frac{1}{2}} D_{0}\right) \frac{d \theta}{f(\theta)}
\end{aligned}
$$

and

$$
I_{3}=\frac{2 \epsilon}{3 \alpha_{1}} \int_{\theta_{1}}^{\theta_{f}} U\left(\theta_{f}\right) U^{-1}(\theta) e^{-\frac{2}{3 \alpha_{1}} \int_{\theta f}^{\theta} \frac{r(\eta)^{\frac{1}{2}}}{|f(\eta)|} d \eta}\left(r^{\frac{1}{2}} D_{0}\right) \frac{d \theta}{f(\theta)} .
$$

From (4.2), (4.8) and $\theta_{1}<\theta<\theta_{f}$ we have

$$
\theta_{0}^{2} \sim C r_{f}^{\frac{3}{2}}
$$

where $C$ depends on $\delta$ and $\theta_{1}$, and

$$
r^{\frac{1}{2}}(\theta) \sim r_{0}^{\frac{1}{2}}\left(\frac{\theta_{0}^{2}}{|f(\theta)|}\right)^{\frac{1}{3}}
$$

We use these relations in order to compute

$$
\int_{\theta_{a}}^{\theta_{b}} \frac{r^{\frac{1}{2}}(\eta)}{|f(\eta)|} d \eta=r_{0}^{\frac{1}{2}} \theta_{0}^{\frac{2}{3}} \int_{\theta_{a}}^{\theta_{b}} \frac{1}{|f(\eta)|^{\frac{4}{3}}} d \eta \sim C r_{f}^{\frac{1}{2}} \int_{\theta_{a}}^{\theta_{b}} \frac{1}{|f(\eta)|^{\frac{4}{3}}} d \eta
$$

If $\theta_{1} \leq \theta_{b}<\theta_{a} \leq \theta_{2}$ then the integral on the right-hand side of (4.23) is bounded and, therefore,

$$
\left|I_{2}\right| \leq \frac{2|\epsilon|}{3 \alpha_{1}} e^{-\frac{2}{3 \alpha_{1}} \int_{\theta f}^{\theta_{1}} \frac{r(\eta) \frac{1}{2}}{f(\eta)} d \eta}\left|I\left(\theta_{0}, \theta_{1}\right)\right| \leq C|\epsilon| .
$$

To estimate $I_{3}$, observe that the integrand in $I_{3}$ is bounded and that the integration is over a bounded interval in $\theta$, so that

$$
\left|I_{3}\right| \leq C|\epsilon|
$$

and this completes the proof of the lemma. $\square$ 
Lemma 4.3 If $\theta_{f}>\theta_{2}$ then $I\left(\theta_{0}, \theta_{f}\right)$ is bounded, i.e.

$$
\left|I\left(\theta_{0}, \theta_{f}\right)\right| \leq C|\epsilon|
$$

for some constant $C$ independent of $\epsilon$.

Proof. We can write

$$
I\left(\theta_{0}, \theta_{f}\right)=\frac{2 \epsilon}{3 \alpha_{1}} U\left(\theta_{f}\right)\left(\int_{\theta_{0}}^{\theta_{1}}+\int_{\theta_{1}}^{\theta_{2}}+\int_{\theta_{2}}^{\theta_{f}}\right) \equiv I_{4}+I_{5}+I_{6}
$$

The terms $I_{4}, I_{5}, I_{6}$ contain the function $e^{-\frac{2}{3 \alpha_{1}} \int_{\theta f}^{\theta} \frac{r(\eta)^{\frac{1}{2}}}{f(\eta)} d \eta}$, whose exponent may be expressed in the form

$$
\frac{2}{3 \alpha_{1}} \int_{\theta f}^{\theta} \frac{r(\eta)^{\frac{1}{2}}}{|f(\eta)|} d \eta=\frac{2}{3 \alpha_{1}} r_{f}^{\frac{1}{2}} f^{\frac{1}{3}}\left(\theta_{f}\right) \int_{\theta f}^{\theta} \frac{1}{|f(\eta)|^{\frac{4}{3}}} d \eta .
$$

If we take into consideration formula (4.8) as well as

$$
f(\theta)=-(\pi-\theta)\left(1+O\left((\pi-\theta)^{2}\right)\right) \text { for } \theta_{2}<\theta<\pi
$$

then we get

$$
\begin{gathered}
\int_{\theta f}^{\theta} \frac{1}{|f(\eta)|^{\frac{4}{3}}} d \eta \sim-3\left(\pi-\theta_{f}\right)^{-\frac{1}{3}}-\frac{3}{5} \theta^{-\frac{5}{3}} \text { if } \theta, \pi-\theta_{f}<<1, \\
\int_{\theta f}^{\theta} \frac{1}{|f(\eta)|^{\frac{4}{3}}} d \eta \sim-3\left(\pi-\theta_{f}\right)^{-\frac{1}{3}} \text { if } \pi-\theta_{f}<<1, \theta>\theta_{1}, \\
\int_{\theta f}^{\theta} \frac{1}{|f(\eta)|^{\frac{4}{3}}} d \eta \sim-3\left(\left(\pi-\theta_{f}\right)^{-\frac{1}{3}}-(\pi-\theta)^{-\frac{1}{3}}\right) \text { if } \pi-\theta_{f}, \pi-\theta<<1 .
\end{gathered}
$$

Hence

$$
\begin{aligned}
I_{4} & \sim \frac{2 \epsilon}{3 \alpha_{1}} C_{1} \int_{\theta_{0}}^{\theta_{1}} U\left(\theta_{f}\right) U^{-1}(\theta) e^{\frac{2}{3 \alpha_{1}} r_{f}^{\frac{1}{2}}\left(\pi-\theta_{f}\right)^{\frac{1}{3}}\left(-3\left(\pi-\theta_{f}\right)^{-\frac{1}{3}}-\frac{3}{5} \theta^{-\frac{5}{3}}\right)}\left(r^{\frac{1}{2}} D_{0}\right) \frac{d \theta}{\theta^{2}}, \\
I_{5} & \sim \frac{2 \epsilon}{3 \alpha_{1}} \int_{\theta_{1}}^{\theta_{2}} U\left(\theta_{f}\right) U^{-1}(\theta) e^{-\frac{2}{3 \alpha_{1}} r_{f}^{\frac{1}{2}}\left(\pi-\theta_{f}\right)^{\frac{1}{3}} \int_{\theta_{f}}^{\theta} \frac{r(\eta) \frac{1}{2}}{|f(\eta)|} d \eta}\left(r^{\frac{1}{2}} D_{0}\right) \frac{d \theta}{f(\theta)}, \\
I_{6} & \sim \frac{2 \epsilon}{3 \alpha_{1}} C_{2} \int_{\theta_{2}}^{\theta_{f}} U\left(\theta_{f}\right) U^{-1}(\theta) e^{\frac{2}{\alpha_{1}} r_{f}^{\frac{1}{2}}\left(\pi-\theta_{f}\right)^{\frac{1}{3}}\left(\left(\pi-\theta_{f}\right)^{-\frac{1}{3}}-(\pi-\theta)^{-\frac{1}{3}}\right)}\left(r^{\frac{1}{2}} D_{0}\right) \frac{d \theta}{\pi-\theta},
\end{aligned}
$$

where $C_{1}, C_{2}$ are constants depending on $\theta_{1}, \theta_{2}, \delta, \alpha_{1}$. In the exact expression for $I_{4}, I_{5}$ and $I_{6}$ we need to replace $f(\theta) \sim-\theta^{2}$ near $\theta=0$ and $f(\theta) \sim-(\pi-\theta)$ near $\theta=\pi$ by their expressions from (4.8) and (4.25). To estimate $I_{5}$ we write

$$
\int_{\theta f}^{\theta} \frac{r(\eta)^{\frac{1}{2}}}{|f(\eta)|} d \eta=\int_{\theta f}^{\theta_{2}} \frac{r(\eta)^{\frac{1}{2}}}{|f(\eta)|} d \eta+\int_{\theta 2}^{\theta} \frac{r(\eta)^{\frac{1}{2}}}{|f(\eta)|} d \eta \quad\left(\theta_{1}<\theta<\theta_{2}\right)
$$


Then

$$
\left|e^{-\frac{2}{3 \alpha_{1}} \int_{\theta f}^{\theta_{2}} \frac{r(\eta)^{\frac{1}{2}}}{|f(\eta)|} d \eta}\right| \leq C .
$$

By the proof of the previous lemma with $\theta_{f}$ replaced by $\theta_{2}$ we then get

$$
\left|I_{5}\right| \leq C|\epsilon| \text {. }
$$

To estimate $I_{4}$ we first drop the terms $O\left(\theta^{2}\right)$ and $O\left((\pi-\theta)^{2}\right)$ in the formulas for $f(\theta)$ in (4.8) and (4.25). Then the exponent in (4.26) is of the form

$$
\begin{gathered}
\frac{1}{\alpha_{1}} r_{f}^{\frac{1}{2}}\left(\pi-\theta_{f}\right)^{\frac{1}{3}}\left(-3\left(\pi-\theta_{f}\right)^{-\frac{1}{3}}-\frac{3}{5} \theta^{-\frac{5}{3}}\right)=-\frac{3}{\alpha_{1}} r_{f}^{\frac{1}{2}}-\frac{3}{5} \frac{1}{\alpha_{1}} r_{f}^{\frac{1}{2}}\left(\pi-\theta_{f}\right)^{\frac{1}{3}} \theta^{-\frac{5}{3}} \\
=-\frac{3}{\alpha_{1}} r_{f}^{\frac{1}{2}}-\mu \theta^{-\frac{5}{3}}
\end{gathered}
$$

where

$$
\mu=\frac{2}{5} \frac{1}{\alpha_{1}} r_{f}^{\frac{1}{2}}\left(\pi-\theta_{f}\right)^{\frac{1}{3}} .
$$

If we expand $I_{4}$ as we did for $I_{1}$ and make the change of variables $\theta=\theta_{1} u^{-1}$, we obtain a linear combination of integrals of the form

$$
\begin{aligned}
& L_{3}\left(\mu, \theta_{f}\right)=C_{1} G\left(\theta_{f}\right) L_{1}\left(\mu \theta_{1}^{-\frac{5}{3}}, \theta_{1}\right), \\
& L_{4}\left(\mu, \theta_{f}\right)=C_{2} G\left(\theta_{f}\right) L_{2}\left(\mu \theta_{1}^{-\frac{5}{3}}, \theta_{1}\right)
\end{aligned}
$$

where $C_{1}, C_{2}$ are functions uniformly bounded by a constant times $|\epsilon|$, and

$$
G\left(\theta_{f}\right)=\left(\pi-\theta_{f}\right)^{ \pm \frac{8}{3} \sqrt{b^{\prime}} i} \quad \text { or } \quad G\left(\theta_{f}\right)=1
$$

(which come from $U\left(\theta_{f}\right)$ ); $\mu \rightarrow 0$ as $\theta_{f} \rightarrow \pi$. We can then prove boundedness for $L_{3}$ and $L_{4}$ in the same way as for $L_{1}, L_{2}$.

When the "O" terms in $f$ are not dropped,we need to break the corresponding integrals as in (4.15), (4.18), and then the resulting extra terms are estimated as in the case of (4.16).

Finally, we examine $I_{6}$ and, for simplicity, again drop the " $O$ " term from $f(\theta)$. If we take into account the formula for $M_{+}$given by (3.15) and make the change of variables $u=\log (\pi-\theta)$, we find that $I_{6}$ is a linear combination of the two integrals

$$
\begin{aligned}
& K_{1}\left(\rho, \theta_{f}\right)=\int_{-\log \left(\pi-\theta_{2}\right)}^{-\log \left(\pi-\theta_{f}\right)} e^{ \pm \frac{8}{3} \sqrt{b^{\prime}} u i} e^{-\rho e^{-\frac{1}{3} u}} d u \\
& K_{2}\left(\rho, \theta_{f}\right)=\int_{-\log \left(\pi-\theta_{2}\right)}^{-\log \left(\pi-\theta_{f}\right)} e^{-u} e^{-\rho e^{-\frac{1}{3} u}} d u
\end{aligned}
$$

with bounded coefficients of the form $|\epsilon|$ times $G\left(\theta_{f}\right)$ as defined in (4.31); here

$$
\rho=\frac{2}{\alpha_{1}} r_{f}^{\frac{1}{2}}\left(\pi-\theta_{f}\right)^{\frac{1}{3}}
$$


The terms $e^{ \pm \frac{8}{3} \sqrt{b} u i}$ and $e^{-u}$ originate from $U^{-1}(\theta)$, and $e^{-\rho e^{-\frac{1}{3} u}}$ comes from

$$
e^{-\frac{2}{\alpha_{1}} r_{f}^{\frac{1}{2}}\left(\pi-\theta_{f}\right)^{\frac{1}{3}}(\pi-\theta)^{-\frac{1}{3}}}=e^{-\rho(\pi-\theta)^{-\frac{1}{3}}}
$$

in (4.28). It remains to establish the boundedness of $K_{j}\left(\rho, \theta_{f}\right)$ as $\rho \rightarrow 0$. In order to do that, we expand $e^{-\rho e^{-\frac{1}{3} u}}$ in powers of $\rho e^{-\frac{1}{3} u}$ :

$$
e^{-\rho e^{-\frac{1}{3} u}}=\sum_{n=0}^{\infty} \frac{(-1)^{n}}{n !} \rho^{n} e^{-\frac{n}{3} u}
$$

and integrate term by term. We obtain then

$$
\begin{aligned}
K_{1}\left(\rho, \theta_{f}\right) & =\sum_{n=0}^{\infty} \frac{(-1)^{n}}{n !} \rho^{n} \int_{-\log \left(\pi-\theta_{2}\right)}^{-\log \left(\pi-\theta_{f}\right)} e^{ \pm \frac{8}{3} \sqrt{b^{\prime}} u i} e^{-\frac{n}{3} u} \\
& =\sum_{n=0}^{\infty} \frac{(-1)^{n}}{n !} \rho^{n}\left[\frac{e^{\left( \pm \frac{8}{3} \sqrt{b^{\prime}} i-\frac{n}{3}\right) u}}{\left( \pm \frac{8}{3} \sqrt{b^{\prime}} i-\frac{n}{3}\right)}\right]_{-\log \left(\pi-\theta_{2}\right)}^{-\log \left(\pi-\theta_{f}\right)}
\end{aligned}
$$

and, therefore,

$$
\begin{aligned}
&\left|K_{1}\left(\rho, \theta_{f}\right)\right| \leq \\
&\left|\sum_{n=0}^{\infty} \frac{(-1)^{n}}{n !} \rho^{n}\left[\frac{e^{\left( \pm \frac{8}{3} \sqrt{b^{\prime}} i-\frac{n}{3}\right) u}}{\left( \pm \frac{8}{3} \sqrt{b^{\prime}} i-\frac{n}{3}\right)}\right]_{-\log \left(\pi-\theta_{2}\right)}^{-\log \left(\pi-\theta_{f}\right)}\right| \leq C\left|\frac{3}{8 \sqrt{b^{\prime}}} \sum_{n=0}^{\infty} \frac{(-1)^{n}}{n !} \rho^{n}\right| \leq C .
\end{aligned}
$$

As for $K_{2}\left(\rho, \theta_{f}\right)$, we immediately have

$$
\left|K_{2}\left(\rho, \theta_{f}\right)\right| \leq\left|\int_{0}^{\infty} e^{-u} e^{-\rho e^{-\frac{1}{3} u}} d u\right| \leq C
$$

and this ends the proof. $\square$

\section{Hölder estimates for the principal term}

In this section we derive a Hölder estimate for the function $I\left(\theta_{0}, \theta_{f}\right)$ (of course, $\theta_{0}=\theta_{0}\left(\theta_{f}\right)$ is a function of $\left.\theta_{f}\right)$, but we shall not indicate this explicitly). As in Section 4 we consider separately the three regions in (4.6) with $\theta_{0}, \theta_{1}, \theta_{2}$ as in (4.7), and it suffices to estimate

$$
\left|I\left(\theta_{0,1}, \theta_{f, 1}\right)-I\left(\theta_{0,2}, \theta_{f, 2}\right)\right|
$$

where $\theta_{f, 1}, \theta_{f, 2}$ both belong to one of these three regions, i.e.,

$$
\begin{aligned}
& \theta_{0}<\theta_{f, 1}<\theta_{f, 2}<\theta_{1}, \\
& \theta_{1}<\theta_{f, 1}<\theta_{f, 2}<\theta_{2}, \\
& \theta_{2}<\theta_{f, 1}<\theta_{f, 2}<\pi .
\end{aligned}
$$

In the sequel we assume that

$$
\frac{r_{f, 1}}{2}<r_{f, 2}<\frac{3 r_{f, 1}}{2}, \frac{\theta_{f, 1}}{2}<\theta_{f, 2}<\frac{3 \theta_{f, 1}}{2} .
$$


Lemma 5.1 In the case (5.1), there holds

$$
\left|I\left(\theta_{0,1}, \theta_{f, 1}\right)-I\left(\theta_{0,2}, \theta_{f, 2}\right)\right| \leq C|\epsilon|\left(\frac{\left|\theta_{f, 1}-\theta_{f, 2}\right|^{\alpha}}{\theta_{f, 2}^{\alpha}}+\frac{\left|r_{f, 1}-r_{f, 2}\right|^{\alpha}}{r_{f, 2}^{\alpha}}\right)
$$

where $C$ is a constant independent of $\epsilon$.

Proof. For simplicity we shall first drop the term $O\left(\theta^{b_{0}}\right)$ in $I\left(\theta_{0}, \theta_{f}\right)$ (see (4.12)). Then from the structure of $I\left(\theta_{0}, \theta_{f}\right)$ (see (4.12) and the subsequent detailed expansion) we see that it suffices to establish (5.5) for the integrals $J=J\left(r_{f}, \theta_{f}\right)$ and $K=K\left(r_{f}, \theta_{f}\right)$ defined in (4.13), (4.14). Clearly,

$$
\begin{aligned}
\Delta J= & \theta_{f, 1}^{-1} \int_{1}^{\frac{\theta_{f, 1}}{\theta_{0,1}}} e^{-\rho_{1}\left(u^{\frac{5}{3}}-1\right)\left(1+O\left(\theta^{2}\right)\right)} u^{-\delta} e^{ \pm \frac{4}{3} \frac{i}{\theta_{f, 1}}(u-1)} d u- \\
& -\theta_{f, 2}^{-1} \int_{1}^{\frac{\theta_{f, 2}}{\theta_{0,2}}} e^{-\rho_{2}\left(u^{\frac{5}{3}}-1\right)\left(1+O\left(\theta^{2}\right)\right)} u^{-\delta} e^{ \pm \frac{4}{3} \frac{i}{\theta_{f, 2}}(u-1)} d u \\
\Delta K= & \int_{1}^{\frac{\theta_{f, 1}}{\theta_{0,2}}} e^{-\rho_{1}\left(u^{\frac{5}{3}}-1\right)\left(1+O\left(\theta^{2}\right)\right)} u^{-\delta} d u \\
& -\int_{1}^{\frac{\theta_{f, 2}}{\theta_{0,1}}} e^{-\rho_{2}\left(u^{\frac{5}{3}}-1\right)\left(1+O\left(\theta^{2}\right)\right)} u^{-\delta} d u
\end{aligned}
$$

where $\delta=\frac{2 b}{3}$ for the first integral and $\delta=\frac{2 b}{3}+1$ for the second integral. Here

$$
\rho_{1}=\frac{2}{5 \alpha_{1}} \frac{r_{f, 1}^{\frac{1}{2}}}{\theta_{f, 1}}, \rho_{2}=\frac{2}{5 \alpha_{1}} \frac{r_{f, 2}^{\frac{1}{2}}}{\theta_{f, 2}}
$$

and $\theta_{0,1}=C r_{f, 1}^{\frac{3}{4}} \theta_{f, 1}, \theta_{0,2}=C r_{f, 2}^{\frac{3}{4}} \theta_{f, 2}$.

We claim that

$$
\left|\rho_{1}-\rho_{2}\right| \leq C \rho_{1}\left(\frac{\left|\theta_{f, 1}-\theta_{f, 2}\right|^{\alpha}}{\theta_{f, 2}^{\alpha}}+\frac{\left|r_{f, 1}-r_{f, 2}\right|^{\alpha}}{r_{f, 2}^{\alpha}}\right)
$$

Indeed, writing

$$
\rho_{1}-\rho_{2}=\frac{3}{5 \alpha_{1}} r_{f, 1}^{\frac{1}{2}}\left(\frac{1}{\theta_{f, 1}}-\frac{1}{\theta_{f, 2}}\right)+\frac{3}{5 \alpha_{1}} \frac{r_{f, 2}^{\frac{1}{2}}}{\theta_{f, 2}} \frac{r_{f, 1}^{\frac{1}{2}}-r_{f, 2}^{\frac{1}{2}}}{r_{f, 2}^{\frac{1}{2}}}
$$

we have

$$
\left|\rho_{1}-\rho_{2}\right| \leq \rho_{1}\left|\frac{\theta_{f, 2}-\theta_{f, 1}}{\theta_{f, 2}}\right|+\rho_{2}\left|\frac{r_{f, 2}-r_{f, 1}}{r_{f, 2}}\right|^{\frac{1}{2}},
$$

and since, by (5.4), $\rho_{2} \leq C \rho_{1}$ and

$$
\left|\frac{\theta_{f, 2}-\theta_{f, 1}}{\theta_{f, 2}}\right| \leq C\left|\frac{\theta_{f, 2}-\theta_{f, 1}}{\theta_{f, 2}}\right|^{\alpha},\left|\frac{r_{f, 2}-r_{f, 1}}{r_{f, 2}}\right|^{\frac{1}{2}} \leq\left|\frac{r_{f, 2}-r_{f, 1}}{r_{f, 2}}\right|^{\alpha} \quad\left(\text { if } \alpha<\frac{1}{2}\right)
$$


(5.9) follows

To estimate $\Delta J$, we first take for simplicity $O\left(\theta^{2}\right) \equiv 0$ and write

$$
\Delta J=\Delta_{1}+\Delta_{2}+\Delta_{3}+\Delta_{4}
$$

where

$$
\begin{gathered}
\Delta_{1}= \\
\theta_{f, 1}^{-1} \int_{1}^{\frac{\theta_{f, 1}}{\theta_{0,1}}} e^{-\rho_{1}\left(u^{\frac{5}{3}}-1\right)} u^{-\delta} e^{ \pm \frac{4}{3} \frac{i}{\theta_{f, 1}}(u-1)} d u-\theta_{f, 1}^{-1} \int_{1}^{\frac{\theta_{f, 2}}{\theta_{0,2}}} e^{-\rho_{1}\left(u^{\frac{5}{3}}-1\right)} u^{-\delta} e^{ \pm \frac{4}{3} \frac{i}{\theta_{f, 1}}(u-1)} d u \\
\Delta_{2}=\left(\theta_{f, 1}^{-1}-\theta_{f, 2}^{-1}\right) \int_{1}^{\frac{\theta_{f, 2}}{\theta_{0,2}}} e^{-\rho_{1}\left(u^{\frac{5}{3}}-1\right)} u^{-\delta} e^{ \pm \frac{4}{3} \frac{i}{\theta_{f, 1}}(u-1)} d u \\
\Delta_{3}=\theta_{f, 2}^{-1} \int_{1}^{\frac{\theta_{f, 2}}{\theta_{0,2}}}\left(e^{-\rho_{1}\left(u^{\frac{5}{3}}-1\right)} u^{-\delta} e^{ \pm \frac{4}{3} \frac{i}{\theta_{f, 1}}(u-1)}-e^{-\rho_{2}\left(u^{\frac{5}{3}}-1\right)} u^{-\delta} e^{ \pm \frac{4}{3} \frac{i}{\theta_{f, 1}}(u-1)}\right) d u \\
\Delta_{4}=\theta_{f, 2}^{-1} \int_{1}^{\frac{\theta_{f, 2}}{\theta_{0,2}}}\left(e^{-\rho_{1}\left(u^{\frac{5}{3}}-1\right)} u^{-\delta} e^{ \pm \frac{4}{3} \frac{i}{\theta_{f, 1}}(u-1)}-e^{-\rho_{1}\left(u^{\frac{5}{3}}-1\right)} u^{-\delta} e^{ \pm \frac{4}{3} \frac{i}{\theta_{f, 2}}(u-1)}\right) d u
\end{gathered}
$$

By (4.2), (4.8) we deduce that

$$
r_{f}^{\frac{3}{2}} \theta_{f}^{2}\left(1+O\left(\theta_{f}^{2}\right)\right)=r_{0}^{\frac{3}{2}} \theta_{0}^{2}\left(1+O\left(\theta_{0}^{2}\right)\right)
$$

and, hence,

$$
r_{f}^{-\frac{3}{4}}=C_{0} \frac{\theta_{f}}{\theta_{0}} \frac{1+O\left(\theta_{f}^{2}\right)}{1+O\left(\theta_{0}^{2}\right)} \quad\left(C_{0} \text { constant }\right)
$$

from which we get

$$
\frac{\theta_{f}}{\theta_{0}}=C_{0}^{-1} r_{f}^{-\frac{3}{4}}\left(1+g\left(\theta_{f}, \theta_{0}\right)\right) \equiv r_{f}^{-\frac{3}{4}} C\left(\theta_{f}, \theta_{0}\right), \quad g \text { smooth }
$$

Setting $C_{1}=C\left(\theta_{f, 1}, \theta_{0,1}\right), C_{2}=C\left(\theta_{f, 2}, \theta_{0,2}\right)$ we then have

$$
\begin{aligned}
& \Delta_{1}=\theta_{f, 1}^{-1} \int_{\frac{\theta_{f, 2}}{\theta_{0,2}}}^{\frac{\theta_{f, 1}}{\theta_{0,1}}} e^{-\rho_{1}\left(u^{\frac{5}{3}}-1\right)} u^{-\delta} e^{ \pm \frac{4}{3} \frac{i}{\theta_{f, 1}}(u-1)} d u \\
& =\theta_{f, 1}^{-1} \int_{C_{1} r_{f, 1}^{-\frac{3}{4}}}^{C_{2} r_{f, 2}^{-\frac{3}{4}}} e^{-\rho_{1}\left(u^{\frac{5}{3}}-1\right)} u^{-\delta} e^{ \pm \frac{4}{3} \frac{i}{\theta_{f, 1}}(u-1)} d u
\end{aligned}
$$

and therefore (we assume, for simplicity, that $C_{2} r_{f, 2}^{-\frac{3}{4}}>C_{1} r_{f, 1}^{-\frac{3}{4}}$ )

$$
\begin{aligned}
\left|\Delta_{1}\right| \leq\left|\theta_{f, 1}^{-1} \int_{C_{1} r_{f, 1}^{-\frac{3}{4}}}^{C_{2} r_{f, 2}^{-\frac{3}{4}}} e^{-\rho_{1}\left(u^{\frac{5}{3}}-1\right)} u^{-\delta} e^{ \pm \frac{4}{3} \frac{i}{\theta_{f, 1}}(u-1)} d u\right| \\
\quad \leq \theta_{f, 1}^{-1}\left|\int_{C_{1} r_{f, 1}^{-\frac{3}{4}}}^{C_{2} r_{f, 2}^{-\frac{3}{4}}} e^{-\rho_{1}\left(u^{\frac{5}{3}}-1\right)} u^{-\delta} d u\right|
\end{aligned}
$$




$$
=\theta_{f, 1}^{-1} e^{\rho_{1}} \int_{C_{1} r_{f, 1}^{-\frac{3}{4}}}^{C_{2} r_{f, 2}^{-\frac{3}{4}}} e^{-\rho_{1} u^{\frac{5}{3}}} u^{-\delta} d u=\frac{3}{5} \theta_{f, 1}^{-1} e^{\rho_{1}} \int_{C_{1}^{\frac{5}{3}} r_{f, 1}^{-\frac{5}{4}}}^{C_{2}^{\frac{5}{3}} r_{f, 2}^{-\frac{5}{4}}} e^{-\rho_{1} v} v^{-\frac{3}{5} \delta} v^{-\frac{2}{5}} d v .
$$

Since $r_{f, 1}$ and $r_{f, 2}$ are small, using the formula

$$
\int_{A}^{B} e^{-\rho v} v^{-\gamma} d v=\left[\frac{1}{\rho} e^{-\rho v} v^{-\gamma}\left(1+O\left(\frac{1}{v}\right)\right]_{A}^{B} \quad \text { for large } A \text { and } B>A,\right.
$$

the right-hand side has an asymptotic behavior whose principal term is

$$
\begin{gathered}
\frac{3}{5} \frac{1}{\rho_{1}} \theta_{f, 1}^{-1} e^{\rho_{1}}\left(e^{-\rho_{1} C_{1}^{\frac{5}{3}} r_{f, 1}^{-\frac{5}{4}}}\left(C_{1}^{\frac{5}{3}} r_{f, 1}^{-\frac{5}{4}}\right)^{-\frac{2}{5}-\frac{3}{5} \delta}-e^{-\rho_{1} C_{2}^{\frac{5}{3}} r_{f, 2}^{-\frac{5}{4}}}\left(C_{2}^{\frac{5}{3}} r_{f, 2}^{-\frac{5}{4}}\right)^{-\frac{2}{5}-\frac{3}{5} \delta}\right) \\
\quad=C \frac{1}{r_{f, 1}^{\frac{1}{2}}} e^{\rho_{1}}\left(e^{-\rho_{1} C_{1}^{\frac{5}{3}} r_{f, 1}^{-\frac{5}{4}}} C_{1}^{-\frac{2}{3}-\delta} r_{f, 1}^{\frac{1}{2}+\frac{3}{4} \delta}-e^{-\rho_{1} C_{2}^{\frac{5}{3}} r_{f, 2}^{-\frac{5}{4}}} C_{2}^{-\frac{2}{3}-\delta} r_{f, 2}^{\frac{1}{2}+\frac{3}{4} \delta}\right) \\
=\Delta_{1,1}+\Delta_{1,2}+\Delta_{1,3}+\Delta_{1,4}
\end{gathered}
$$

where

$$
\begin{aligned}
& \Delta_{1,1}=C C_{1}^{-\frac{2}{3}-\delta} \frac{1}{r_{f, 1}^{\frac{1}{2}}} e^{-\rho_{1} C_{1}^{\frac{5}{3}} r_{f, 2}^{-\frac{5}{4}}} e^{\rho_{1}}\left(r_{f, 1}^{\frac{1}{2}+\frac{3}{4} \delta}-r_{f, 2}^{\frac{1}{2}+\frac{3}{4} \delta}\right) \\
& \Delta_{1,2}=C C_{1}^{-\frac{2}{3}-\delta} \frac{1}{r_{f, 1}^{\frac{1}{2}}} r_{f, 1}^{\frac{1}{2}+\frac{3}{4} \delta} e^{\rho_{1}}\left(e^{-\rho_{1} C_{1}^{-\frac{5}{3}} r_{f, 1}^{-\frac{5}{4}}}-e^{-\rho_{1} C_{1}^{\frac{5}{3}} r_{f, 2}^{-\frac{5}{4}}}\right), \\
& \Delta_{1,3}=C \frac{1}{r_{f, 1}^{\frac{1}{2}}} r_{f, 1}^{\frac{1}{2}+\frac{3}{4} \delta} e^{\rho_{1}} e^{-\rho_{1} C_{1}^{\frac{5}{3}} r_{f, 1}^{-\frac{5}{4}}}\left(C_{1}^{-\frac{2}{3}-\delta}-C_{2}^{-\frac{2}{3}-\delta}\right) \\
& \Delta_{1,4}=C C_{2}^{-\frac{2}{3}-\delta} \frac{1}{r_{f, 1}^{\frac{1}{2}}} e^{\rho_{1}} r_{f, 1}^{\frac{1}{2}+\frac{3}{4} \delta}\left(e^{-\rho_{1} C_{1}^{\frac{5}{3}} r_{f, 2}^{-\frac{5}{4}}}-e^{-\rho_{1} C_{2}^{\frac{5}{3}} r_{f, 2}^{-\frac{5}{4}}}\right)
\end{aligned}
$$

Using the inequality

$$
1-e^{-x} \leq C(\gamma) x^{\gamma} \text { for } 0 \leq \gamma \leq 1
$$

and the mean value theorem, we find that

$$
\Delta_{1,1} \leq\left|r_{f, 1}^{\frac{1}{2}+\frac{3}{4} \delta}-r_{f, 2}^{\frac{1}{2}+\frac{3}{4} \delta}\right| \leq C\left|r_{f, 1}-r_{f, 2}\right|^{\alpha}
$$

and

$$
\begin{aligned}
\Delta_{1,2} & \leq r_{f, 1}^{\frac{3}{4} \delta} e^{\rho_{1}} e^{-\rho_{1} C^{-\frac{5}{3}} r_{f, 1}^{-\frac{5}{4}}}\left|1-e^{-\rho_{1} C_{1}^{-\frac{5}{3}}\left(r_{f, 2}^{-\frac{5}{4}}-r_{f, 1}^{-\frac{5}{4}}\right)}\right| \\
& \leq C r_{f, 1}^{\frac{3}{4} \delta} e^{\rho_{1}} e^{-\rho_{1} C^{-\frac{5}{3}} r_{f, 1}^{-\frac{5}{4}}} \rho_{1}^{\gamma}\left|r_{f, 2}^{-\frac{5}{4}}-r_{f, 1}^{-\frac{5}{4}}\right|^{\gamma} \\
& \leq C r_{f, 1}^{\frac{3}{4} \delta} e^{\rho_{1}} e^{-\rho_{1} C^{-\frac{5}{3}} r_{f, 1}^{-\frac{5}{4}}} \rho_{1}^{\gamma} r_{f, 1}^{\left(-1-\frac{5}{4}\right) \gamma}\left|r_{f, 2}-r_{f, 1}\right|^{\frac{5}{4} \gamma} \leq C\left|r_{f, 2}-r_{f, 1}\right|^{\alpha},
\end{aligned}
$$


provided $\alpha$ is small enough.

Next by (5.12) and the smoothness of $g(\theta)$ we find that

$$
\begin{aligned}
& \left|\Delta_{1,3}\right| \leq C\left(\left|r_{f, 1}-r_{f, 2}\right|^{\alpha}+\left|\theta_{f, 1}-\theta_{f, 2}\right|^{\alpha}\right) \\
& \left|\Delta_{1,4}\right| \leq C\left(\left|r_{f, 1}-r_{f, 2}\right|^{\alpha}+\left|\theta_{f, 1}-\theta_{f, 2}\right|^{\alpha}\right) .
\end{aligned}
$$

Combining the above estimates we conclude that

$$
\left|\Delta_{1}\right| \leq C\left(\left|r_{f, 1}-r_{f, 2}\right|^{\alpha}+\left|\theta_{f, 1}-\theta_{f, 2}\right|^{\alpha}\right) .
$$

Next we turn to $\Delta_{2}$, which is easily estimated by

$$
\begin{gathered}
\Delta_{2} \leq\left|\theta_{f, 1}^{-1}-\theta_{f, 2}^{-1}\right|\left|\int_{1}^{\frac{\theta_{f, 2}}{\theta_{0,2}}} e^{-\rho_{1}\left(u^{\frac{5}{3}}-1\right)} u^{-\delta} e^{ \pm \frac{4}{3} \frac{i}{\theta_{f, 1}}(u-1)} d u\right| \\
\leq C\left|\theta_{f, 1}^{-1}-\theta_{f, 2}^{-1}\right|\left|\theta_{f, 2}\right| \leq C \frac{\left|\theta_{f, 1}-\theta_{f, 2}\right|^{\alpha}}{\left|\theta_{f, 1}\right|^{\alpha}} .
\end{gathered}
$$

To estimate $\Delta_{3}$ we write

$$
\begin{gathered}
\Delta_{3}=\theta_{f, 1}^{-1} \int_{1}^{\frac{\theta_{f, 2}}{\theta_{0,2}}}\left(e^{-\rho_{1}\left(u^{\frac{5}{3}}-1\right)}-e^{-\rho_{2}\left(u^{\frac{5}{3}}-1\right)}\right) u^{-\delta} e^{ \pm \frac{4}{3} \frac{i}{\theta_{f, 1}}(u-1)} d u \\
=\theta_{f, 1}^{-1} \int_{1}^{\infty}\left(e^{-\rho_{1}\left(u^{\frac{5}{3}}-1\right)}-e^{-\rho_{2}\left(u^{\frac{5}{3}}-1\right)}\right) u^{-\delta} e^{ \pm \frac{4}{3} \frac{i}{\theta_{f, 1}}(u-1)} d u \\
-\theta_{f, 1}^{-1} \int_{\frac{\theta_{f, 2}}{\theta_{0,2}}}^{\infty}\left(e^{-\rho_{1}\left(u^{\frac{5}{3}}-1\right)}-e^{-\rho_{2}\left(u^{\frac{5}{3}}-1\right)}\right) u^{-\delta} e^{ \pm \frac{4}{3} \frac{i}{\theta_{f, 1}}(u-1)} d u \equiv \Delta_{3,1}+\Delta_{3,2} .
\end{gathered}
$$

We may change of contour of integration $u \rightarrow 1 \pm \theta_{f, 1} e^{\frac{3 \pi}{10} i} s$ to get

$$
\begin{aligned}
\Delta_{3,1}= & \theta_{f, 1}^{-1} \int_{1}^{\infty}\left(e^{-\rho_{1}\left(u^{\frac{5}{3}}-1\right)}-e^{-\rho_{2}\left(u^{\frac{5}{3}}-1\right)}\right) u^{-\delta} e^{ \pm \frac{4}{3} \frac{i}{\theta_{f, 1}}(u-1)} d u \\
= & e^{\frac{3 \pi}{10} i} \int_{0}^{\infty}\left(1 \pm \theta_{f, 1} e^{\frac{3 \pi}{10} i} s\right)^{-\delta} e^{\frac{4}{3}\left(\cos \frac{3 \pi}{10}\right) s i} e^{-\frac{4}{3}\left(\sin \frac{3 \pi}{10}\right) s} \\
& \times\left(e^{-\rho_{1}\left(\left(1 \pm \theta_{f, 1} e^{\frac{3 \pi}{10} i} s\right)^{\frac{5}{3}}-1\right)}-e^{-\rho_{2}\left(\left(1 \pm \theta_{f, 1} e^{\frac{3 \pi}{10} i} s\right)^{\frac{5}{3}}-1\right)}\right) d s
\end{aligned}
$$

We then deduce, using again (5.13) (with $\gamma=2 \alpha$ )

$$
\begin{aligned}
\left|\Delta_{3,1}\right| & \leq C \int_{0}^{\infty}\left|1 \pm \theta_{f, 1} e^{\frac{3 \pi}{10} i} s\right|^{-\delta+\frac{5}{3} \gamma} e^{-\frac{4}{3}\left(\sin \frac{3 \pi}{10}\right) s}\left|e^{-\rho_{1}\left(\left(1 \pm \theta_{f, 1} e^{\frac{3 \pi}{10} i} s\right)^{\frac{5}{3}}-1\right)}\right|\left|\rho_{2}-\rho_{1}\right|^{\gamma} d s \\
& \leq C \theta_{f, 1}^{-\delta+\frac{5}{3} \gamma}\left|\rho_{2}-\rho_{1}\right|^{\gamma} \leq C \theta_{f, 1}^{-\delta+\frac{5}{3} \gamma} \rho_{1}^{\gamma}\left(\frac{\left|r_{f, 1}-r_{f, 2}\right|^{\alpha}}{r_{f, 1}^{\alpha}}+\frac{\left|\theta_{f, 1}-\theta_{f, 2}\right|^{\alpha}}{\theta_{f, 1}^{\alpha}}\right) .
\end{aligned}
$$


As for $\Delta_{3,2}$, setting $\widetilde{\rho}=\min \left(\rho_{1}, \rho_{2}\right)$ we have

$$
\begin{gathered}
\left|\Delta_{3,2}\right| \leq\left|\theta_{f, 1}^{-1} \int_{\frac{\theta_{f, 2}}{\theta_{0,2}}}^{\infty}\left(e^{-\rho_{1}\left(u^{\frac{5}{3}}-1\right)}-e^{-\rho_{2}\left(u^{\frac{5}{3}}-1\right)}\right) u^{-\delta} e^{ \pm \frac{4}{3} \frac{i}{\theta_{f, 1}}(u-1)} d u\right| \\
\leq\left|\theta_{f, 1}^{-1} \int_{C_{2} r_{f, 2}^{-\frac{3}{4}}}^{\infty}\right| e^{-\rho_{1}\left(u^{\frac{5}{3}}-1\right)}-e^{-\rho_{2}\left(u^{\frac{5}{3}}-1\right)}\left|u^{-\delta} d u\right| \\
\leq\left|\theta_{f, 1}^{-1} \int_{C_{2} r_{f, 2}^{-\frac{3}{4}}}^{\infty} e^{-\widetilde{\rho}\left(u^{\frac{5}{3}}-1\right)}\right| \rho_{1}-\rho_{2}\left|\left(u^{\frac{5}{3}}-1\right) u^{-\delta} d u\right| \quad \text { (by the mean value theorem) } \\
\leq C\left|\theta_{f, 1}^{-1} e^{\widetilde{\rho}} \int_{C_{2} r_{f, 2}^{-\frac{3}{4}}}^{\infty} e^{-\widetilde{\rho} u^{\frac{5}{3}}} u^{\frac{5}{3}-\delta} d u\right|\left|\rho_{1}-\rho_{2}\right| \\
=C \theta_{f, 1}^{-1} e^{\widetilde{\rho} \widetilde{\rho}\left(-\frac{8}{3}+\frac{3}{5} \delta\right)}\left|\int_{C_{2} \widetilde{\rho}^{\frac{3}{5}} r_{f, 2}^{-\frac{3}{4}}}^{\infty} e^{-v^{\frac{5}{3}}} v^{\frac{5}{3}-\delta} d v\right|\left|\rho_{1}-\rho_{2}\right| \quad\left(v=\widetilde{\rho}^{\frac{5}{3}} u\right) .
\end{gathered}
$$

Using the estimate

$$
\int_{A}^{\infty} e^{-v^{\frac{5}{3}}} v^{\gamma} d v \leq e^{-A^{\frac{5}{3}}} A^{\gamma} \quad(\text { for any } A>1)
$$

the inequalities in (5.4), (5.9), and

$$
\frac{\widetilde{\rho}}{r_{f, 1}^{\frac{5}{4}}} \leq C \frac{r_{f, 1}^{\frac{1}{2}}}{\theta_{f, 1} r_{f, 1}^{\frac{5}{4}}} \leq \frac{C}{\theta_{f, 1} r_{f, 1}^{\frac{3}{4}}}
$$

we conclude that

$$
\left|\Delta_{3,2}\right| \leq C\left(\frac{\left|r_{f, 1}-r_{f, 2}\right|^{\alpha}}{r_{f, 1}^{\alpha}}+\frac{\left|\theta_{f, 1}-\theta_{f, 2}\right|^{\alpha}}{\theta_{f, 1}^{\alpha}}\right) .
$$

Since the same estimate was also obtained for $\left|\Delta_{3,1}\right|$, we conclude that

$$
\left|\Delta_{3}\right| \leq C\left(\frac{\left|r_{f, 1}-r_{f, 2}\right|^{\alpha}}{r_{f, 1}^{\alpha}}+\frac{\left|\theta_{f, 1}-\theta_{f, 2}\right|^{\alpha}}{\theta_{f, 1}^{\alpha}}\right) .
$$

Finally we can write

$$
\begin{aligned}
\Delta_{4}= & \theta_{f, 2}^{-1} \int_{1}^{\infty} e^{-\rho_{1}\left(u^{\frac{5}{3}}-1\right)} u^{-\delta}\left(e^{ \pm \frac{4}{3} \frac{i}{\theta_{f, 1}}(u-1)}-e^{ \pm \frac{4}{3} \frac{i}{\theta_{f, 2}}(u-1)}\right) d u \\
& -\theta_{f, 2}^{-1} \int_{\frac{\theta_{f, 2}}{\theta_{0}}}^{\infty} e^{-\rho_{1}\left(u^{\frac{5}{3}}-1\right)} u^{-\delta}\left(e^{ \pm \frac{4}{3} \frac{i}{\theta_{f, 1}}(u-1)}-e^{ \pm \frac{4}{3} \frac{i}{\theta_{f, 2}}(u-1)}\right) d u \\
= & \Delta_{4,1}+\Delta_{4,2}
\end{aligned}
$$

By a change of contour of integration,

$$
\Delta_{4,1}=e^{\frac{3 \pi}{10} i} \theta_{f, 2}^{-1}
$$


$\times \int_{0}^{\infty}\left(\begin{array}{c}\theta_{f, 1}\left(1+\theta_{f, 1} e^{\frac{3 \pi}{10} i} s\right)^{-\delta} e^{-\rho_{1}\left(\left(1+\theta_{f, 1} e^{\frac{3 \pi}{10} i} s\right)^{\frac{5}{3}}-1\right)}- \\ -\theta_{f, 2}\left(1+\theta_{f, 2} e^{\frac{3 \pi}{10} i} s\right)^{-\delta} e^{-\rho_{1}\left(\left(1+\theta_{f, 2} e^{\frac{3 \pi}{10} i} s\right)^{\frac{5}{3}}-1\right)}\end{array}\right) e^{\frac{4}{3}\left(\cos \frac{3 \pi}{10}\right) s i} e^{-\frac{4}{3}\left(\sin \frac{3 \pi}{10}\right) s} d s$

Decomposing the expression in the large parenthesis into

$$
\begin{gathered}
\left(1+\theta_{f, 1} e^{\frac{3 \pi}{10} i} s\right)^{-\delta} e^{-\rho_{1}\left(\left(1+\theta_{f, 1} e^{\frac{3 \pi}{10} i} s\right)^{\frac{5}{3}}-1\right)}\left[\theta_{f, 1}-\theta_{f, 2}\right] \\
+\theta_{f, 2}\left[\left(1+\theta_{f, 2} e^{\frac{3 \pi}{10} i} s\right)^{-\delta}-\left(1+\theta_{f, 1} e^{\frac{3 \pi}{10} i} s\right)^{-\delta}\right] e^{-\rho_{1}\left(\left(1+\theta_{f, 2} e^{\frac{3 \pi}{10} i} s\right)^{\frac{5}{3}}-1\right)} \\
+\theta_{f, 2}\left(1+\theta_{f, 1} e^{\frac{3 \pi}{10} i} s\right)^{-\delta}\left[e^{-\rho_{1}\left(\left(1+\theta_{f, 2} e^{\frac{3 \pi}{10} i} s\right)^{\frac{5}{3}}-1\right)}-e^{-\rho_{2}\left(\left(1+\theta_{f, 1} e^{\frac{3 \pi}{10} i} s\right)^{\frac{5}{3}}-1\right)}\right]
\end{gathered}
$$

and correspondingly decomposing $\Delta_{4,1}$, one can estimate each of the resulting integrals in same way we did in previous cases, and obtain the same estimate for $\Delta_{4,1}$ as for $\Delta_{3}$; indeed, the third integral can be treated as $\Delta_{3,1}$, the first by using the $L^{\infty}$ bound as in Section 5 , and the second by using the mean value theorem and $L^{\infty}$ bound as in Section 5 .

Next, $\Delta_{4,2}$ can be estimated as $\Delta_{3,2}$ and thus we conclude that $\left|\Delta_{4}\right|$ is bounded by the right hand side of (5.5). Since the same was already proved for $\Delta_{1}, \Delta_{2}$ and $\Delta_{3}$, the estimate (5.5) holds for $\Delta J$.

So far we have assumed that $O\left(\theta^{2}\right) \equiv 0$ in (5.6). In the general case we need to add and substract a term as in (4.15) or (4.18); the resulting extra integrals that need to be estimated can easily be treated analogously to the term $R$ in (4.15) using the fact that $O\left(\theta^{2}\right)$ is actually a smooth function of $\theta^{2}$

We proceed to estimate for $\Delta K$ (defined in (5.7)). Taking for simplicity $O\left(\theta^{2}\right) \equiv 0$, we can write

$$
\Delta K=\Delta_{1}+\Delta_{2}
$$

where

$$
\begin{gathered}
\Delta_{1}=\int_{1}^{\frac{\theta_{f, 1}}{\theta_{0,1}}} e^{-\rho_{1}\left(u^{\frac{5}{3}}-1\right)} u^{-\delta} d u-\int_{1}^{\frac{\theta_{f, 2}}{\theta_{0,2}}} e^{-\rho_{1}\left(u^{\frac{5}{3}}-1\right)} u^{-\delta} d u \\
=\int_{\frac{\theta_{f, 2}}{\theta_{0,1}}}^{\frac{\theta_{f, 1}}{\theta_{0,2}}} e^{-\rho_{1}\left(u^{\frac{5}{3}}-1\right)} u^{-\delta} d u \\
\Delta_{2}=\int_{1}^{\frac{\theta_{f, 2}}{\theta_{0,2}}}\left(e^{-\rho_{1}\left(u^{\frac{5}{3}}-1\right)}-e^{-\rho_{2}\left(u^{\frac{5}{3}}-1\right)}\right) u^{-\delta} d u .
\end{gathered}
$$

$\Delta_{1}$ may be estimated as the first term at the right hand side of (5.10), and

$$
\begin{gathered}
\left|\Delta_{2}\right| \leq \int_{1}^{\infty}\left|e^{-\rho_{1}\left(u^{\frac{5}{3}}-1\right)}-e^{-\rho_{2}\left(u^{\frac{5}{3}}-1\right)}\right| u^{-\delta} d u \\
=\frac{3}{5} \int_{0}^{\infty}\left|e^{-\rho_{1} v}-e^{-\rho_{2} v}\right|(1+v)^{-\frac{2}{5}-\frac{3}{5} \delta} d v \\
\leq C \int_{0}^{\infty}\left|\rho_{1}-\rho_{2}\right|^{\gamma} v^{\gamma} e^{-\rho_{1} v}(1+v)^{-\frac{2}{5}-\frac{3}{5} \delta} d v \leq C\left|\rho_{1}-\rho_{2}\right|^{\gamma} \frac{1}{1+\rho_{1}} \\
\leq \frac{1}{r_{f, 1}^{\alpha}}\left|r_{f, 1}-r_{f, 2}\right|^{\alpha}+\frac{1}{\theta_{f, 1}^{\alpha}}\left|\theta_{f, 1}-\theta_{f, 2}\right|^{\alpha}
\end{gathered}
$$


where we have chosen $\gamma=2 \alpha$. Thus $|\Delta K|$ is bounded by the right hand side of (5.5). This completes the proof of Lemma 5.1 provided the term $O\left(\theta^{b_{0}}\right) \equiv 0$. Since $O\left(\theta^{b_{0}}\right)$ is a smooth function of $\theta^{b_{0}}$ (see Remark 3.1) the Hölder estimate of the term resulting from $O\left(\theta^{b_{0}}\right)$ can be obtained by just minor changes in some of the previous arguments (cf. the end of the proof of Lemma 4.1).

Lemma 5.2 In the case (5.2), there holds

$$
\left|I\left(\theta_{0,1}, \theta_{f, 1}\right)-I\left(\theta_{0,2}, \theta_{f, 2}\right)\right| \leq C|\epsilon|\left(\frac{\left|\theta_{f, 1}-\theta_{f, 2}\right|^{\alpha}}{\theta_{f, 2}^{\alpha}}+\frac{\left|r_{f, 1}-r_{f, 2}\right|^{\alpha}}{r_{f, 2}^{\alpha}}\right)
$$

where $C$ is a constant independent of $\epsilon$.

Since the proof involves estimates of integrals which already appeared in the proof of Lemma 5.1, we omit the details.

Lemma 5.3 In the case (5.3), there holds

$$
\left|I\left(\theta_{0,1}, \theta_{f, 1}\right)-I\left(\theta_{0,2}, \theta_{f, 2}\right)\right| \leq C|\epsilon|\left(\frac{\left|\theta_{f, 1}-\theta_{f, 2}\right|^{\alpha}}{\left(\pi-\theta_{f, 2}\right)^{\alpha}}+\frac{\left|r_{f, 1}-r_{f, 2}\right|^{\alpha}}{r_{f, 2}^{\alpha}}\right)
$$

where $C$ is a constant independent of $\epsilon$.

Proof. For simplicity we shall drop the error term $g$ in (5.12), i.e., we take $g \equiv 0$; the term involving $g$ can be estimated more easily using the smoothness of $g$.

We first estimate the Hölder coefficient of the term $I_{4}$ of $I$ (see (4.24)). To do that we need to estimate

$$
\begin{gathered}
\Delta J=L_{3}\left(\mu_{1}, \theta_{f, 1}\right)-L_{3}\left(\mu_{2}, \theta_{f, 2}\right), \\
\Delta K=L_{4}\left(\mu_{1}, \theta_{f, 1}\right)-L_{4}\left(\mu_{2}, \theta_{f, 2}\right)
\end{gathered}
$$

where $L_{3}$ and $L_{4}$ are defined by (4.29) and (4.30), respectively, and

$$
\mu_{1}=\frac{2}{5} \frac{1}{\alpha_{1}} r_{f, 1}^{\frac{1}{2}}\left(\pi-\theta_{f, 1}\right)^{\frac{1}{3}}, \quad \mu_{2}=\frac{2}{5} \frac{1}{\alpha_{1}} r_{f, 2}^{\frac{1}{2}}\left(\pi-\theta_{f, 2}\right)^{\frac{1}{3}}
$$

We can write

$$
\begin{aligned}
\Delta J & =C_{1}\left[\left(G\left(\theta_{f, 1}\right)-G\left(\theta_{f, 2}\right)\right) L_{1}\left(\mu_{1}, \theta_{1}\right)+G\left(\theta_{f, 2}\right)\left(L_{1}\left(\mu_{1}, \theta_{1}\right)-L_{1}\left(\mu_{2}, \theta_{1}\right)\right)\right] \\
\Delta K & =C_{2}\left[\left(G\left(\theta_{f, 1}\right)-G\left(\theta_{f, 2}\right)\right) L_{2}\left(\mu_{1}\right)+G\left(\theta_{f, 2}\right)\left(L_{1}\left(\mu_{1}\right)-L_{1}\left(\mu_{2}\right)\right)\right]
\end{aligned}
$$

where $G\left(\theta_{f}\right)$ is defined in (4.31). If we use the fact that

$$
\left|G\left(\theta_{f, 1}\right)-G\left(\theta_{f, 2}\right)\right| \leq \frac{\left|\theta_{f, 1}-\theta_{f, 2}\right|^{\alpha}}{\left(\pi-\theta_{f, 1}\right)^{\alpha}}
$$


and the Hölder estimate derived above for $L_{1}$ (in the proof of Lemma 5.1), we obtain

$$
\begin{aligned}
& |\Delta J| \leq C\left(\frac{\left|r_{f, 1}-r_{f, 2}\right|^{\alpha}}{r_{f, 1}^{\alpha}}+\frac{\left|\theta_{f, 1}-\theta_{f, 2}\right|^{\alpha}}{\left(\pi-\theta_{f, 1}\right)^{\alpha}}\right), \\
& |\Delta K| \leq C\left(\frac{\left|r_{f, 1}-r_{f, 2}\right|^{\alpha}}{r_{f, 1}^{\alpha}}+\frac{\left|\theta_{f, 1}-\theta_{f, 2}\right|^{\alpha}}{\left(\pi-\theta_{f, 1}\right)^{\alpha}}\right) .
\end{aligned}
$$

To estimate the Hölder constant of the term $I_{6}$ in (4.24), it suffices to do that for the functions $K_{1}\left(\rho, \theta_{f}\right)$ and $K_{2}\left(\rho, \theta_{f}\right)$ defined by (4.32)-(4.34) Thus we need to estimate.

$$
\Delta K_{1} \equiv \int_{-\log \left(\pi-\theta_{2}\right)}^{-\log \left(\pi-\theta_{f, 1}\right)} e^{ \pm \frac{8}{3} \sqrt{b^{\prime}} u i} e^{-\rho_{1} e^{-\frac{1}{3} u}} d u-\int_{-\log \left(\pi-\theta_{2}\right)}^{-\log \left(\pi-\theta_{f, 2}\right)} e^{ \pm \frac{8}{3} \sqrt{b^{\prime}} u i} e^{-\rho_{2} e^{-\frac{1}{3} u}} d u
$$

and

$$
\Delta K_{2} \equiv \int_{-\log \left(\pi-\theta_{2}\right)}^{-\log \left(\pi-\theta_{f, 1}\right)} e^{-u} e^{-\rho_{1} e^{-\frac{1}{3} u}} d u-\int_{-\log \left(\pi-\theta_{2}\right)}^{-\log \left(\pi-\theta_{f, 2}\right)} e^{-u} e^{-\rho_{2} e^{-\frac{1}{3} u}} d u .
$$

To estimate $\Delta K_{1}$ we write

$$
\Delta K_{1}=\Delta_{1}+\Delta_{2}
$$

where

$$
\begin{aligned}
& \Delta_{1}=\int_{-\log \left(\pi-\theta_{f, 2}\right)}^{-\log \left(\pi-\theta_{f, 1}\right)} e^{ \pm \frac{8}{3} \sqrt{b^{\prime}} u i} e^{-\rho_{1} e^{-\frac{1}{3} u} d u} \\
& \Delta_{2}=\int_{-\log \left(\pi-\theta_{2}\right)}^{-\log \left(\pi-\theta_{f, 2}\right)} e^{ \pm \frac{8}{3} \sqrt{b^{\prime}} u i}\left(e^{-\rho_{1} e^{-\frac{1}{3} u}}-e^{-\rho_{2} e^{-\frac{1}{3} u}}\right) d u .
\end{aligned}
$$

Then

$$
\begin{aligned}
\left|\Delta_{1}\right| & \leq \int_{-\log \left(\pi-\theta_{f, 2}\right)}^{-\log \left(\pi-\theta_{f, 1}\right)} e^{-\rho_{1} e^{-\frac{1}{3} u}} d u \leq \log \left(\pi-\theta_{f, 2}\right)-\log \left(\pi-\theta_{f, 1}\right) \\
& \leq \log \left(1+\frac{\theta_{f, 1}-\theta_{f, 2}}{\pi-\theta_{f, 1}}\right) \leq \frac{\left|\theta_{f, 2}-\theta_{f, 1}\right|}{\left(\pi-\theta_{f, 1}\right)} \leq \frac{\left|\theta_{f, 2}-\theta_{f, 1}\right|^{\alpha}}{\left(\pi-\theta_{f, 1}\right)^{\alpha}}
\end{aligned}
$$

Also

$$
\begin{gathered}
\left|\Delta_{2}\right| \leq\left|\int_{-\log \left(\pi-\theta_{2}\right)}^{-\log \left(\pi-\theta_{f, 2}\right)} e^{ \pm \frac{8}{3} \sqrt{b^{\prime}} u i}\left(e^{-\rho_{2} e^{-\frac{1}{3} u}}-e^{-\rho_{2} e^{-\frac{1}{3} u}}\right) d u\right| \\
\leq C\left|\int_{0}^{\infty}\left(1-e^{-\left(\rho_{2}-\rho_{1}\right) e^{-\frac{1}{3} u}}\right) d u\right| \leq C\left|\int_{0}^{\infty} e^{-\frac{1}{3} u} d u\right|\left|\rho_{2}-\rho_{1}\right| \\
\leq C\left(\left|r_{f, 1}-r_{f, 2}\right|^{\alpha}+\left|\theta_{f, 1}-\theta_{f, 2}\right|^{\alpha}\right) .
\end{gathered}
$$

Combining these estimates we conclude that $\left|\Delta K_{1}\right|$ is bounded by the right hand side of (5.15). $\left|\Delta K_{2}\right|$ can be estimated in a similar way, and thus (5.14) holds for $I_{6}$, Finally, the Hölder estimate for $I_{5}$ (with the same Hölder coefficient as for $I_{4}, I_{5}$ ) can be established as in Lemma 5.1. 


\section{$6 L^{\infty}$ estimates for the perturbation}

In this section we estimate the term $J\left(\theta_{0}, \theta_{f}\right)$ in (4.5). Let us introduce, for brevity, the function

$$
W(r, \theta)=r^{\frac{1}{2}} \widetilde{D}(r, \theta) .
$$

As in Section 5, we consider separately the three different regions in (4.6)

Lemma 6.1 If $\theta_{0}<\theta_{f}<\theta_{1}$ then

$$
\left|J\left(\theta_{0}, \theta_{f}\right)\right| \leq C|\epsilon| \sup |\widetilde{D}|
$$

where $C$ is a constant independent of $\epsilon$.

Proof. Following the proof of Lemma 4.1 with $r^{\frac{1}{2}} D_{0}$ replaced by $W$, we arrive at the following analogs of (4.13), (4.14):

$$
\begin{aligned}
& L_{1}\left[W ; r_{f}, \theta_{f}\right]=\theta_{f}^{-1} \int_{1}^{\frac{\theta_{f}}{\theta 1}} e^{-\rho\left(u^{\frac{5}{3}}-1\right)} u^{-\delta} e^{\frac{4}{3} \theta_{f}^{-1}(u-1) i} W\left(r_{f} u^{\frac{4}{3}}, \theta_{f} u^{-1}\right) d u(6.1) \\
& L_{2}\left[W ; r_{f}, \theta_{f}\right]=\int_{1}^{\frac{\theta_{f}}{\theta 1}} e^{-\rho\left(u^{\frac{5}{3}}-1\right)} u^{-\delta} e^{\frac{4}{3} \theta_{f}^{-1}(u-1) i} W\left(r_{f} u^{\frac{4}{3}}, \theta_{f} u^{-1}\right) d u
\end{aligned}
$$

where $\delta=\frac{2 b}{3}$ in (6.1), $\delta=\frac{2 b}{3}+1$ in (6.2), and

$$
|W(r, \theta)| \leq r^{\frac{1}{2}} \sup |\widetilde{D}|
$$

for simplicity we have dropped the term $O\left(\theta^{2}\right)$ in the exponents. Since the singularity of $W$ is weaker than the singularity of $r^{\frac{1}{2}} D_{0}$, we do not need in the present case to change the contour of integration (for the integral $L_{1}$ ) and we may directly estimate, for $j=1,2$,

$$
\begin{aligned}
& \left|L_{j}\left[G ; r_{f}, \theta_{f}\right]\right| \leq \theta_{f}^{-1} \int_{1}^{\infty} e^{-\rho\left(u^{\frac{5}{3}}-1\right)} u^{-\delta}\left|W\left(r_{f} u^{\frac{4}{3}}, \theta_{f} u^{-1}\right)\right| d u \\
& \leq C r_{f}^{\frac{1}{2}} \theta_{f}^{-1} \int_{1}^{\infty} e^{-\rho\left(u^{\frac{5}{3}}-1\right)} u^{\frac{2}{3}-\delta} d u=C \rho e^{\rho} \int_{1}^{\infty} e^{-\rho u^{\frac{5}{3}}} u^{\frac{2}{3}-\delta} d u \\
& =C e^{\rho} \rho^{\frac{3}{5} \delta} \int_{\rho^{\frac{3}{5}}}^{\infty} e^{-u^{\frac{5}{3}}} u^{\frac{2}{3}-\delta} d u \leq C .
\end{aligned}
$$

Finally, the term with $O\left(\theta^{2}\right)$ can be estimated as in Lemma 4.1.

Lemma 6.2 If $\theta_{1}<\theta_{f}<\theta_{2}$ then

$$
\left|J\left(\theta_{0}, \theta_{f}\right)\right| \leq C|\epsilon| \sup |\widetilde{D}|
$$

where $C$ is a constant independent of $\epsilon$. 
Proof. We just need to reproduce the arguments of Lemma 4.2 combined with the same remark as in the proof of Lemma 6.1 regarding the weak singularity of $W$.

Lemma 6.3 If $\theta_{2}<\theta_{f}<\pi$ then

$$
\left|J\left(\theta_{0}, \theta_{f}\right)\right| \leq C|\epsilon| \sup |\widetilde{D}|
$$

where $C$ is a constant independent of $\epsilon$.

Proof. As in the proof of Lemma 4.3 we derive the formulas (4.26), (4.27), (4.28) with $D_{0}$ substituted by $\widetilde{D}$. We follow the proof of Lemma 4.3 and proceed to estimate the integrals $I_{4}, I_{5}, I_{6}$ with $D_{0}$ replaced by $\widetilde{D}$. The estimate on $I_{4}$ is reduced to estimating expressions of the form

$$
\begin{aligned}
& L_{3}\left[W ; r_{f}, \theta_{f}\right]=C_{1} G\left(\theta_{f}\right) L_{1}\left[W ; \mu \theta_{1}^{-\frac{5}{3}}, \theta_{1}\right], \\
& L_{4}\left[W ; r_{f}, \theta_{f}\right]=C_{2} G\left(\theta_{f}\right) L_{2}\left[W ; \mu \theta_{1}^{-\frac{5}{3}}, \theta_{1}\right]
\end{aligned}
$$

and this can be done as in the proof of Lemma 6.1. The estimate for $I_{5}$ is immediate, as before. Finally, the estimate of $I_{6}$ is reduced to estimating terms of the form

$$
\begin{gathered}
K_{1}\left[W ; r_{f}, \theta_{f}\right]=\int_{-\log \left(\pi-\theta_{2}\right)}^{-\log \left(\pi-\theta_{f}\right)} e^{ \pm \frac{8}{3} \sqrt{b^{\prime}} u i} W\left(r_{f} e^{-\frac{4}{3} u},\left(\pi-\theta_{f}\right) e^{u}\right) e^{-\rho e^{-\frac{1}{3} u}} d u \\
K_{2}\left[W ; r_{f}, \theta_{f}\right]=\int_{-\log \left(\pi-\theta_{2}\right)}^{-\log \left(\pi-\theta_{f}\right)} e^{-u} W\left(r_{f} e^{-\frac{4}{3} u},\left(\pi-\theta_{f}\right) e^{u}\right) e^{-\rho e^{-\frac{1}{3} u}} d u
\end{gathered}
$$

Since

$$
\begin{aligned}
\left|K_{j}\right| & \leq \int_{-\log \left(\pi-\theta_{2}\right)}^{-\log \left(\pi-\theta_{f}\right)}\left|W\left(r_{f} e^{-\frac{4}{3} u},\left(\pi-\theta_{f}\right) e^{u}\right)\right| e^{-\rho e^{-\frac{1}{3} u}} d u \\
& \leq \int_{-\log \left(\pi-\theta_{2}\right)}^{-\log \left(\pi-\theta_{f}\right)} r_{f}^{\frac{1}{2}} e^{-\frac{2}{3} u} e^{-\rho e^{-\frac{1}{3} u}} d u \leq C(j=1,2)
\end{aligned}
$$

the proof is complete.

\section{Hölder estimates for the perturbation}

In order to obtain Hölder estimates of the integral coming from the perturbation, we assume that $W$ satisfies

$$
\begin{aligned}
\left|W\left(r_{f}, \theta_{f}\right)\right| & \leq C r_{f}^{\frac{1}{2}} \\
\left|W\left(r_{f, 1}, \theta_{f}\right)-W\left(r_{f, 2}, \theta_{f}\right)\right| & \leq C r_{f, 1}^{\frac{1}{2}-\alpha}\left|r_{f, 1}-r_{f, 2}\right|^{\alpha} \\
\left|W\left(r_{f}, \theta_{f, 1}\right)-W\left(r_{f}, \theta_{f, 2}\right)\right| & \leq C r_{f}^{\frac{1}{2}} \theta_{f, 1}^{-\alpha}\left|\theta_{f, 1}-\theta_{f, 2}\right|^{\alpha} \\
\left|W\left(r_{f}, \theta_{f, 1}\right)-W\left(r_{f}, \theta_{f, 2}\right)\right| & \leq C r_{f}^{\frac{1}{2}}\left(\pi-\theta_{f, 1}\right)^{-\alpha}\left|\theta_{f, 1}-\theta_{f, 2}\right|^{\alpha}
\end{aligned}
$$


here $r_{f, j}, \theta_{f, j}$ are as in (5.4), and $\alpha>0$ and small enough.

Recall, by (4.5), that

$$
J\left(\theta_{0}, \theta_{f}\right)=\frac{2 \epsilon}{3 \alpha_{1}} \int_{\theta_{0}}^{\theta_{f}} U\left(\theta_{f}\right) U^{-1}(\theta) e^{-\frac{2}{3 \alpha_{1}} \int_{\theta f}^{\theta} \frac{r(\theta)^{\frac{1}{2}}}{|f(\theta)|} d \theta} W\left(r_{f}, \theta_{f}\right) \frac{d \theta}{f(\theta)} .
$$

Lemma 7.1 If $0<\theta_{f, 1}, \theta_{f, 2}<\theta_{1}$ then

$$
\left|J\left(\theta_{0}, \theta_{f, 1}\right)-J\left(\theta_{0}, \theta_{f, 2}\right)\right| \leq C|\epsilon|\left(r_{f, 1}^{-\alpha}\left|r_{f, 1}-r_{f, 2}\right|^{\alpha}+\theta_{f, 1}^{-\alpha}\left|\theta_{f, 1}-\theta_{f, 2}\right|^{\alpha}\right) .
$$

where $C$ is a constant independent of $\epsilon$.

Proof. As in Section 6, it is sufficient to prove this estimate for the functions $L_{1}$ and $L_{2}$ corresponding to $W$. Let us for simplicity drop the term with $O\left(\theta^{b_{0}}\right)$, which can be estimated, in fact, more easily. We claim that the functions $L_{j}$ $(j=1,2)$ defined in $(6.1),(6.2)$ satisfy

$$
\left|L_{j}\left[W ; r_{f, 1}, \theta_{f, 1}\right]-L_{j}\left[W ; r_{f, 2}, \theta_{f, 2}\right]\right| \leq C\left(r_{f, 1}^{-\alpha}\left|r_{f, 1}-r_{f, 2}\right|^{\alpha}+\theta_{f, 1}^{-\alpha}\left|\theta_{f, 1}-\theta_{f, 2}\right|^{\alpha}\right) .
$$

To prove it, we proceed analogously to the proof of Lemma 5.1 and consider first the case $j=1$. We write

$$
L_{1}\left[W ; r_{f, 1}, \theta_{f, 1}\right]=\widetilde{L_{1}}\left[W ; r_{f, 1}, \theta_{f, 1}\right]+\widetilde{\widetilde{L_{1}}}\left[W ; r_{f, 1}, \theta_{f, 1}\right]
$$

where $\widetilde{L_{1}}$ is defined as $L_{1}$ but with integration from $u=0$ to $u=\infty$. We first derive the Hölder estimate for $\widetilde{L_{1}}$. We can write

$$
\begin{gathered}
\widetilde{L_{1}}\left[W ; r_{f, 1}, \theta_{f, 1}\right]-\widetilde{L_{1}}\left[W ; r_{f, 2}, \theta_{f, 2}\right] \\
=\theta_{f, 1}^{-1} \int_{1}^{\infty} e^{-\rho_{1}\left(u^{\frac{5}{3}}-1\right)} e^{\frac{4}{3} \theta_{f, 1}^{-1}(u-1) i} u^{-\delta} W\left(r_{f, 1} u^{\frac{4}{3}}, \theta_{f, 1} u^{-1}\right) d u \\
-\theta_{f, 2}^{-1} \int_{1}^{\infty} e^{-\rho_{2}\left(u^{\frac{5}{3}}-1\right)} e^{\frac{4}{3} \theta_{f, 2}^{-1}(u-1) i} u^{-\delta} W\left(r_{f, 2} u^{\frac{4}{3}}, \theta_{f, 2} u^{-1}\right) d u \\
=\int_{0}^{\infty} e^{\frac{4}{3} u i}\left\{\begin{array}{l}
e^{-\rho_{1}\left(\left(1+\theta_{f, 1} u\right)^{\frac{5}{3}}-1\right)}\left(1+\theta_{f, 1} u\right)^{-\delta} W\left(r_{f, 1}\left(1+\theta_{f, 1} u\right)^{\frac{4}{3}}, \frac{\theta_{f, 1}}{1+\theta_{f, 1} u}\right) \\
-e^{-\rho_{2}\left(\left(1+\theta_{f, 2} u\right)^{\frac{5}{3}}-1\right)}\left(1+\theta_{f, 2} u\right)^{-\delta} W\left(r_{f, 2}\left(1+\theta_{f, 2} u\right)^{\frac{4}{3}}, \frac{\theta_{f, 2}}{1+\theta_{f, 2} u}\right)
\end{array}\right\} d u
\end{gathered}
$$

Setting

$$
\begin{aligned}
& a_{1}=r_{f, 1}\left(1+\theta_{f, 1} u\right)^{\frac{4}{3}}, a_{2}=r_{f, 2}\left(1+\theta_{f, 2} u\right)^{\frac{4}{3}} \\
& b_{1}=\frac{\theta_{f, 1}}{1+\theta_{f, 1} u} \quad, \quad b_{2}=\frac{\theta_{f, 2}}{1+\theta_{f, 2} u} .
\end{aligned}
$$

We then have

$$
\left|\widetilde{L_{1}}\left[W ; r_{f, 1}, \theta_{f, 1}\right]-\widetilde{L_{1}}\left[W ; r_{f, 2}, \theta_{f, 2}\right]\right| \leq \Delta_{1}+\Delta_{2}+\Delta_{3}+\Delta_{4}
$$


where

$$
\begin{aligned}
& \Delta_{1} \leq\left|\int_{0}^{\infty} e^{\frac{4}{3} u i} e^{-\rho_{1}\left(\left(1+\theta_{f, 1} u\right)^{\frac{5}{3}}-1\right)}\left(1+\theta_{f, 1} u\right)^{-\delta}\left\{W\left(a_{1}, b_{1}\right)-W\left(a_{2}, b_{2}\right)\right\} d u\right| \\
& \Delta_{2} \leq\left|\int_{0}^{\infty} e^{\frac{4}{3} u i}\left(1+\theta_{f, 1} u\right)^{-\delta}\left\{e^{-\rho_{1}\left(\left(1+\theta_{f, 1} u\right)^{\frac{5}{3}}-1\right)}-e^{-\rho_{1}\left(\left(1+\theta_{f, 2} u\right)^{\frac{5}{3}}-1\right)}\right\} W\left(a_{2}, b_{2}\right) d u\right|, \\
& \Delta_{3} \leq\left|\int_{0}^{\infty} e^{\frac{4}{3} u i}\left(1+\theta_{f, 1} u\right)^{-\delta}\left\{e^{-\rho_{1}\left(\left(1+\theta_{f, 2} u\right)^{\frac{5}{3}}-1\right)}-e^{-\rho_{2}\left(\left(1+\theta_{f, 2} u\right)^{\frac{5}{3}}-1\right)}\right\} W\left(a_{2}, b_{2}\right) d u\right|, \\
& \Delta_{4} \leq\left|\int_{0}^{\infty} e^{\frac{4}{3} u i} e^{-\rho_{1}\left(\left(1+\theta_{f, 2} u\right)^{\frac{5}{3}}-1\right)}\left\{\left(1+\theta_{f, 1} u\right)^{-\delta}-\left(1+\theta_{f, 2} u\right)^{-\delta}\right\} W\left(a_{2}, b_{2}\right) d u\right| .
\end{aligned}
$$

To estimate (7.6) we note that

$$
\begin{gathered}
\left|W\left(a_{1}, b_{1}\right)-W\left(a_{2}, b_{2}\right)\right| \\
\leq\left|W\left(r_{f, 1}\left(1+\theta_{f, 1} u\right)^{\frac{4}{3}}, \frac{\theta_{f, 1}}{1+\theta_{f, 1} u}\right)-W\left(r_{f, 1}\left(1+\theta_{f, 2} u\right)^{\frac{4}{3}}, \frac{\theta_{f, 2}}{1+\theta_{f, 2} u}\right)\right| \\
+\left|W\left(r_{f, 1}\left(1+\theta_{f, 1} u\right)^{\frac{4}{3}}, \frac{\theta_{f, 2}}{1+\theta_{f, 2} u}\right)-W\left(r_{f, 2}\left(1+\theta_{f, 2} u\right)^{\frac{4}{3}}, \frac{\theta_{f, 2}}{1+\theta_{f, 2} u}\right)\right| \equiv \Gamma_{1}+\Gamma_{2}
\end{gathered}
$$

and

$$
\begin{gathered}
\Gamma_{1} \leq r_{f, 1}^{\frac{1}{2}}\left(1+\theta_{f, 1} u\right)^{\frac{2}{3}-\alpha}\left|\left(1+\theta_{f, 1} u\right)^{\frac{4}{3}}-\left(1+\theta_{f, 2} u\right)^{\frac{4}{3}}\right|^{\alpha} \\
+r_{f, 1}^{\frac{1}{2}}\left(1+\theta_{f, 1} u\right)^{\frac{2}{3}}\left(\frac{\theta_{f, 1}}{1+\theta_{f, 1} u}\right)^{-\alpha}\left|\frac{\theta_{f, 1}}{1+\theta_{f, 1} u}-\frac{\theta_{f, 2}}{1+\theta_{f, 2} u}\right|^{\alpha} \leq \\
\leq C r_{f, 1}^{\frac{1}{2}}\left(1+\theta_{f, 1} u\right)^{\frac{2}{3}-\frac{2}{3} \alpha} u^{\alpha}\left|\theta_{f, 1}-\theta_{f, 2}\right|^{\alpha}+C r_{f, 1}^{\frac{1}{2}}\left(1+\theta_{f, 1} u\right)^{\frac{2}{3}-\alpha} \frac{\left|\theta_{f, 1}-\theta_{f, 2}\right|^{\alpha}}{\theta_{f, 1}^{\alpha}} \\
\Gamma_{2} \leq r_{f, 1}^{\frac{1}{2}-\alpha}\left(1+\theta_{f, 1} u\right)^{\frac{2}{3}-\alpha}\left|r_{f, 1}\left(1+\theta_{f, 1} u\right)^{\frac{4}{3}}-r_{f, 2}\left(1+\theta_{f, 2} u\right)^{\frac{4}{3}}\right|^{\alpha} \\
\leq C r_{f, 1}^{\frac{1}{2}-\alpha}\left(1+\theta_{f, 1} u\right)^{\frac{2}{3}+\frac{1}{3} \alpha}\left|r_{f, 1}-r_{f, 2}\right|^{\alpha}+C r_{f, 1}^{\frac{1}{2}}\left(1+\theta_{f, 1} u\right)^{\frac{2}{3}-\frac{2}{3} \alpha} u^{\alpha}\left|\theta_{f, 1}-\theta_{f, 2}\right|^{\alpha} .
\end{gathered}
$$

If we introduce the change of variabl

$$
\left(1+\theta_{f, 1} u\right)^{\frac{5}{3}}-1=v
$$

we obtain

$$
\begin{gathered}
\Delta_{1} \leq C r_{f, 1}^{\frac{1}{2}} \theta_{f, 1}^{-\alpha-1}\left|\theta_{f, 1}-\theta_{f, 2}\right|^{\alpha} \int_{0}^{\infty} e^{-\rho_{1} v}(v+1)^{\frac{2}{5}-\frac{2}{5} \alpha-\frac{3}{5} \delta}\left((1+v)^{\frac{3}{5}}-1\right)^{\alpha} \frac{d v}{(1+v)^{\frac{2}{5}}} \\
+C r_{f, 1}^{\frac{1}{2}} \theta_{f, 1}^{-1} \frac{\left|\theta_{f, 1}-\theta_{f, 2}\right|^{\alpha}}{\theta_{f, 1}^{\alpha}} \int_{0}^{\infty} e^{-\rho_{1} v}(1+v)^{\frac{2}{5}-\frac{3}{5} \alpha-\frac{3}{5} \delta} \frac{d v}{(1+v)^{\frac{2}{5}}}
\end{gathered}
$$




$$
+C r_{f, 1}^{\frac{1}{2}-\alpha} \theta_{f, 1}^{-1}\left|r_{f, 1}-r_{f, 2}\right|^{\alpha} \int_{0}^{\infty} e^{-\rho_{1} v}(1+v)^{\frac{2}{5}+\frac{1}{5} \alpha-\frac{3}{5} \delta} \frac{d v}{(1+v)^{\frac{2}{5}}}
$$

Since each of the three integrals is bounded by

$$
\begin{gathered}
\int_{0}^{\infty} e^{-\rho_{1} v}(1+v)^{\frac{2}{5}+\frac{1}{5} \alpha-\frac{3}{5} \delta} \frac{d v}{(1+v)^{\frac{2}{5}}} \leq C_{1} \int_{0}^{1} e^{-\rho_{1} v} d v+C_{2} \int_{1}^{\infty} e^{-\rho_{1} v} v^{\frac{1}{5} \alpha-\frac{3}{5} \delta} d v \\
\leq C_{1} \rho_{1}^{-1}+C_{2} \rho_{1}^{-1-\frac{1}{5} \alpha+\frac{3}{5} \delta}, \text { if } \frac{1}{5} \alpha-\frac{3}{5} \delta<0 \text { (i.e., if } \alpha<3 \delta \text { ) }
\end{gathered}
$$

we conclude, upon recalling (5.8), that

$$
\Delta_{1} \leq C\left(\theta_{f, 1}^{-\alpha}\left|\theta_{f, 1}-\theta_{f, 2}\right|^{\alpha}+r_{f, 1}^{-\alpha}\left|r_{f, 1}-r_{f, 2}\right|^{\alpha}\right) .
$$

To estimate $\Delta_{2}$ we use the mean value theorem and (5.13) to get

$$
\begin{aligned}
& \left|e^{-\rho_{1}\left(\left(1+\theta_{f, 1} u\right)^{\frac{5}{3}}-1\right)}-e^{-\rho_{1}\left(\left(1+\theta_{f, 2} u\right)^{\frac{5}{3}}-1\right)}\right| \\
\leq & C e^{-\rho_{1}\left(\left(1+\theta_{f, 1} u\right)^{\frac{5}{3}}-1\right)}\left|\rho_{1}\left(\left(1+\theta_{f, 1} u\right)^{\frac{5}{3}}-1\right)-\rho_{1}\left(\left(1+\theta_{f, 2} u\right)^{\frac{5}{3}}-1\right)\right|^{\alpha} \\
\leq & C e^{-\rho_{1}\left(\left(1+\theta_{f, 1} u\right)^{\frac{5}{3}}-1\right)}\left(1+\theta_{f, 1} u\right)^{\frac{2}{3} \alpha} \rho_{1}^{\alpha} u^{\alpha}\left|\theta_{f, 1}-\theta_{f, 2}\right|^{\alpha} .
\end{aligned}
$$

Recalling, by (7.1) that

$$
\left|W\left(r_{f, 2}\left(1+\theta_{f, 2} u\right)^{\frac{4}{3}}, \frac{\theta_{f, 2}}{1+\theta_{f, 2} u}\right)\right| \leq r_{f, 2}^{\frac{1}{2}}\left(1+\theta_{f, 2} u\right)^{\frac{2}{3}}
$$

and changing variable as in (7.10), we find that

$\Delta_{2} \leq C r_{f, 2}^{\frac{1}{2}} \rho_{1}^{\alpha} \theta_{f, 1}^{-\alpha-1}\left|\theta_{f, 1}-\theta_{f, 2}\right|^{\alpha} \int_{0}^{\infty} e^{-\rho_{1} v}\left((1+v)^{\frac{3}{5}}-1\right)^{\frac{2}{3} \alpha}(v+1)^{-\frac{1}{5}+\frac{2}{5} \alpha-\frac{3}{5} \delta} d v$ and the last integral is bounded by $C \rho_{1}^{-1}$. Consequently,

$$
\Delta_{2} \leq C \theta_{f, 1}^{-\alpha}\left|\theta_{f, 1}-\theta_{f, 2}\right|^{\alpha}
$$

In order to estimate $\Delta_{3}$, we use the mean value theorem to conclude that

$$
\begin{aligned}
& \left|e^{-\rho_{1}\left(\left(1+\theta_{f, 2} u\right)^{\frac{5}{3}}-1\right)}-e^{-\rho_{2}\left(\left(1+\theta_{f, 2} u\right)^{\frac{5}{3}}-1\right)}\right| \\
= & e^{-\rho_{2}\left(\left(1+\theta_{f, 2} u\right)^{\frac{5}{3}}-1\right)}\left|\left(1+\theta_{f, 2} u\right)^{\frac{5}{3}}-1\right|^{\alpha}\left|\rho_{1}-\rho_{2}\right|^{\alpha}
\end{aligned}
$$

and substitute this into (7.8) to get

$$
\Delta_{3} \leq C r_{f}^{\frac{1}{2}} \theta_{f, 1}^{-1}\left|\rho_{1}-\rho_{2}\right|^{\alpha} \int_{0}^{\infty} e^{-\rho_{2} v} v^{\alpha}(v+1)^{-\frac{2}{5}-\frac{3}{5} \delta} d v
$$


But the last integral is bounded by

$$
\int_{0}^{\infty} e^{-\rho_{2} v} v^{\alpha}(v+1)^{-\frac{2}{5}} d v \leq C \rho_{2}^{-1-\alpha}
$$

and therefore

$$
\Delta_{3} \leq C \theta_{f, 1}^{-\alpha}\left|\theta_{f, 1}-\theta_{f, 2}\right|^{\alpha}
$$

We finally estimate $\Delta_{4}$. By the mean value theorem

$$
\begin{gathered}
\left|\left(1+\theta_{f, 1} u\right)^{-\delta}-\left(1+\theta_{f, 2} u\right)^{-\delta}\right| \leq\left(1+\theta_{f, 1} u\right)^{-\delta-1}\left|\theta_{f, 1}-\theta_{f, 2}\right| u \\
=C \frac{\theta_{f, 1} u}{\left(1+\theta_{f, 1} u\right)^{1+\delta}} \frac{\left|\theta_{f, 1}-\theta_{f, 2}\right|}{\theta_{f, 1}} \leq C \frac{\left|\theta_{f, 1}-\theta_{f, 2}\right|^{\alpha}}{\theta_{f, 1}^{\alpha}}
\end{gathered}
$$

and, by the change of variable $v=u^{\frac{5}{3}}-1$,

$$
\int_{1}^{\infty} e^{-\rho_{1}\left(u^{\frac{5}{3}}-1\right)} u^{\frac{2}{3}} d u=\int_{0}^{\infty} e^{-\rho_{1} v} d v=\rho_{1}^{-1}
$$

Consequently,

$$
\begin{gathered}
\Delta_{4} \leq C r_{f}^{\frac{1}{2}} \frac{\left|\theta_{f, 1}-\theta_{f, 2}\right|^{\alpha}}{\theta_{f, 1}} \int_{0}^{\infty} e^{-\rho_{1}\left(\left(1+\theta_{f, 2} u\right)^{\frac{5}{3}}-1\right)}\left(1+\theta_{f, 2} u\right)^{\frac{2}{3}} d u \\
\leq C \frac{r_{f}^{\frac{1}{2}}}{\theta_{f, 1}} \rho_{1}^{-1}\left|\theta_{f, 1}-\theta_{f, 2}\right|^{\alpha} \leq C\left|\theta_{f, 1}-\theta_{f, 2}\right|^{\alpha} .
\end{gathered}
$$

Combining the above estimates we get

$$
\begin{gathered}
\left|\widetilde{L_{1}}\left[W ; r_{f, 1}, \theta_{f, 1}\right]-\widetilde{L_{1}}\left[W ; r_{f, 2}, \theta_{f, 2}\right]\right| \\
\leq C\left(\theta_{f, 1}^{-\alpha}\left|\theta_{f, 1}-\theta_{f, 2}\right|^{\alpha}+r_{f, 1}^{-\alpha}\left|r_{f, 1}-r_{f, 2}\right|^{\alpha}\right) .
\end{gathered}
$$

The same estimate can be derived, in fact more simply, for $\widetilde{\widetilde{L_{1}}}$ and thus it holds also for $L_{1}$. Note that the only difference between $L_{1}$ and $L_{2}$ is the complex exponent which appears in $L_{1}$. Since the exponent is bound by $1, L_{2}$ can be estimated exactly as $L_{1}$.

Lemma 7.2 If $\theta_{1} \leq \theta_{f, 1}, \theta_{f, 2} \leq \theta_{2}$ then

$$
\left|J\left(\theta_{0}, \theta_{f, 1}\right)-J\left(\theta_{0}, \theta_{f, 2}\right)\right| \leq C|\epsilon|\left(r_{f, 1}^{-\alpha}\left|r_{f, 1}-r_{f, 2}\right|^{\alpha}+\left|\theta_{f, 1}-\theta_{f, 2}\right|^{\alpha}\right)
$$

where $C$ is a constant independent of $|\epsilon|$.

The proof is similar to that of Lemma 7.1.

Lemma 7.3 If $\theta_{2} \leq \theta_{f, 1}, \theta_{f, 2} \leq \pi$ then

$$
\left|J\left(\theta_{0}, \theta_{f, 1}\right)-J\left(\theta_{0}, \theta_{f, 1}\right)\right| \leq C|\epsilon|\left(r_{f, 1}^{-\alpha}\left|r_{f, 1}-r_{f, 2}\right|^{\alpha}+\left(\pi-\theta_{f, 1}\right)^{-\alpha}\left|\theta_{f, 1}-\theta_{f, 2}\right|^{\alpha}\right)
$$

where $C$ is a constant independent of $\epsilon$. 
Proof. The proof is obtained by combining arguments used in Lemma7.1. It will be enough to consider the most delicate case where $\theta_{f}$ is near $\pi$.

As before (cf. proof of Lemma 6.3), it suffices to consider the integrals $K_{1}\left[W ; r_{f}, \theta_{f}\right]$ and $K_{2}\left[W ; r_{f}, \theta_{f}\right]$ defined in (6.5) and (6.6).

Lemma 7.4 For $j=1,2$ there holds

$$
\begin{gathered}
\left|K_{j}\left[W ; r_{f, 2}, \theta_{f, 2}\right]-K_{j}\left[W ; r_{f, 1}, \theta_{f, 1}\right]\right| \\
\leq C\left(r_{f, 1}^{-\alpha}\left|r_{f, 1}-r_{f, 2}\right|^{\alpha}+\left(\pi-\theta_{f, 1}\right)^{-\alpha}\left|\theta_{f, 1}-\theta_{f, 2}\right|^{\alpha}\right) .
\end{gathered}
$$

Proof. We may perform similar decompositions as for $L_{1}$ and $L_{2}$. The "worse" term belongs to $K_{1}$ and it is of the form

$$
\Delta=\int_{-\log \left(\pi-\theta_{f, 1}\right)}^{-\log \left(\pi-\theta_{f, 2}\right)} e^{ \pm \frac{8}{3} \sqrt{b^{\prime}} u i} W\left(r_{f} e^{-\frac{4}{3} u},\left(\pi-\theta_{f}\right) e^{u}\right) e^{-\rho e^{-\frac{1}{3} u}} d u
$$

Breaking up the integral as we did in the case of $\widetilde{L_{1}}$ and using the assumptions (7.1), (7.2), (7.4), we find that

$$
|\Delta| \leq C\left|\log \left(\pi-\theta_{f, 1}\right)-\log \left(\pi-\theta_{f, 2}\right)\right| \leq \frac{\left|\theta_{f, 1}-\theta_{f, 2}\right|^{\alpha}}{\left|\pi-\theta_{f, 2}\right|^{\alpha}} .
$$

The estimates for all the other terms follow the same steps as in previous lemmas and we therefore omit the details. $\square$

\section{The final estimates for the hyperbolic problem}

We shall summarize the estimates derived in Sections 4-7 in terms of norms introduced in [6], [7], [9], [10] and some new norms specially adapted to our problem. We divide $\Sigma$ into two rectangles,

$$
\begin{aligned}
& \Sigma_{1}=\{(x, y) ;-N<x<N, 0<y<1\}, \\
& \Sigma_{2}=\{(x, y) ;-N<x<N,-1<y<0\},
\end{aligned}
$$

and denote a variable point by $X=(x, y)$.

For any small $\delta>0$, let

$$
A_{1}=B_{\delta}(0,1) \cap \Sigma, A_{2}=B_{\delta}(0,-1) \cap \Sigma
$$

and

$$
\rho(X)=\left\{\begin{array}{c}
\delta^{-1}|X-(0,1)| \text { if } X \in A_{1} \\
\delta^{-1}|X-(0,-1)| \text { if } X \in A_{2} \\
1 \quad \text { if } R \backslash\left(A_{1} \cup A_{2}\right)
\end{array}\right.
$$

Definition 8.1 We denote by $C_{s}^{n+\alpha}(\Sigma)$ the set of functions $f: \Sigma \rightarrow \mathbb{R}$ such that

$$
\|f\|_{C_{s}^{n+\alpha}(\Sigma)} \equiv \sum_{|m| \leq n} \sup _{X \in \Sigma}\left(\rho^{|m|-s}(X)\left|D^{m} f\right|\right)
$$




$$
+\sum_{|m|=n} \sup _{X \in \Sigma}\left(\rho^{\alpha+n-s}(X) \sup _{X^{\prime} \in K(X)} \frac{\left|D_{X}^{n} f(X)-D_{X^{\prime}}^{n} f\left(X^{\prime}\right)\right|}{\left|X-X^{\prime}\right|^{\alpha}}\right)<\infty,
$$

where $X=(x, y), X^{\prime}=\left(x^{\prime}, y^{\prime}\right), K(X)=\left\{X^{\prime}:\left|X-X^{\prime}\right| \leq \frac{1}{2} \rho(X)\right\}$. Similarly we define $C_{s}^{n+\alpha}\left(\Sigma_{1}\right)$ and $C_{s}^{n+\alpha}\left(\Sigma_{2}\right)$.

We shall also need to work with functions for which the Hölder condition in (8.1) is allowed to degenerate near $y=1$ or $y=-1$. Such functions are introduced in the next definition.

Definition 8.2 Let $g(r)$ be a positive $C^{\infty}\left(\mathbb{R}^{+}\right)$function with $g^{\prime}(r)>0$, such that

$$
g(r)=\left\{\begin{array}{c}
|\log r| \quad \text { if } r<\frac{1}{2} \\
r \quad \text { if } r>1
\end{array}\right.
$$

and let $h(\theta)$ be a $C^{\infty}(0, \pi)$ function with

$$
h(\theta)=\left\{\begin{array}{c}
\log \theta \quad \text { if } \theta<\frac{\pi}{3} \\
\log (\pi-\theta) \quad \text { if } \theta>\frac{2 \pi}{3}
\end{array}\right.
$$

We denote by $\stackrel{\circ}{C}_{s}^{n+\alpha}\left(\Sigma_{1}\right)$ the set of functions $f: \Sigma_{1} \rightarrow \mathbb{R}$ such that

$$
\begin{gathered}
\|f\|_{C_{s}^{\circ n+\alpha}\left(\Sigma_{1}\right)} \equiv \sum_{|m| \leq n} \sup \left(\sup _{X \in \Sigma_{1}}\left(\rho^{|m|-s}(X)\left|D^{m} f\right|\right)\right) \\
+\sum_{|m|=n} \sup _{X \in \Sigma_{1}}\left\{\rho^{n-s}(X) \sup _{X^{\prime} \in \widetilde{K}(X)} \frac{\left|D_{X}^{n} f(X)-D_{X^{\prime}}^{n} f\left(X^{\prime}\right)\right|}{\left|g(r)-g\left(r^{\prime}\right)\right|^{\alpha}+\left|h(\theta)-h\left(\theta^{\prime}\right)\right|^{\alpha}}\right\}<\infty,
\end{gathered}
$$

where $X=(r, \theta)$ and $X^{\prime}=\left(r^{\prime}, \theta^{\prime}\right)$ are the polar coordinates of $X$ and $X^{\prime}$ around $(0,1), \widetilde{K}(X)$ is a region in the neighborhood of $X$ defined by

$$
\widetilde{K}(X)=\left\{\left(r^{\prime}, \theta^{\prime}\right): \frac{r^{\prime}}{2}<r<\frac{3 r^{\prime}}{2}, \frac{\theta^{\prime}}{2}<\theta<\frac{3 \theta^{\prime}}{2}\right\}
$$

and $D_{X}$ denotes both $\partial_{r}$ and $r^{-1} \partial_{\theta}$. Similarly we define the space $\stackrel{\circ}{C}_{s}^{n+\alpha}\left(\Sigma_{2}\right)$.

Remark 8.1. Note that in (8.1), if $X$ is near a vertex, then

$$
\begin{aligned}
\left|X-X^{\prime}\right|^{2}=r^{2}+r^{\prime 2}- & 2 r r^{\prime} \cos \left(\theta_{1}-\theta_{2}\right)=\left(r-r^{\prime}\right)^{2}+r r^{\prime}\left(1-\cos \left(\theta_{1}-\theta_{2}\right)\right) \\
& \sim\left(r-r^{\prime}\right)^{2}+\frac{1}{2} r r^{\prime}\left(\theta_{1}-\theta_{2}\right)^{2}
\end{aligned}
$$

and, since

$$
\frac{r}{2}<r^{\prime}<\frac{3 r}{2} \quad \text { if } X^{\prime} \in K(X)
$$

we have

$$
\frac{\rho^{\alpha}(X)}{\left|X-X^{\prime}\right|^{\alpha}} \sim \frac{\left(\frac{\rho^{\alpha}}{r^{\alpha}}\right) r^{\alpha}}{\left[\left(r-r^{\prime}\right)^{2}+\frac{r^{2}}{2}\left(\theta_{1}-\theta_{2}\right)^{2}\right]^{\frac{\alpha}{2}}}
$$


which is bounded from above and below by a constant times

$$
\left[\left(\frac{r-r^{\prime}}{r}\right)^{\alpha}+\frac{1}{2}\left(\frac{r^{\prime}}{r}\right)^{\alpha}\left(\theta_{1}-\theta_{2}\right)^{\alpha}\right]^{-1} .
$$

The same is true if $X$ is not near the vertex. Since

$$
g(r)-g\left(r^{\prime}\right) \sim C \frac{\left(r-r^{\prime}\right)}{r_{0}} \text { for some } \frac{r}{2}<r_{0}<\frac{3 r}{2}
$$

where $C$ is uniformly positive and uniformly bounded, we see that the norm in the space $C_{s}^{n+\alpha}\left(\Sigma_{1}\right)$ is equivalent to

$$
\begin{gathered}
\|f\|_{C_{s}^{n+\alpha}\left(\Sigma_{1}\right)} \equiv \sum_{|m| \leq n} \sup _{X \in \Sigma_{1}}\left(\rho^{|m|-s}(X)\left|D^{m} f\right|\right) \\
+\sum_{|m|=n} \sup _{X \in \Sigma_{1}} \sup _{X^{\prime} \in K(X)} \frac{\left|\rho^{n-s}(X) D_{X}^{n} f(X)-\rho^{n-s}\left(X^{\prime}\right) D_{X^{\prime}}^{n} f\left(X^{\prime}\right)\right|}{\left|g(r)-g\left(r^{\prime}\right)\right|^{\alpha}+r^{\alpha} \rho^{-\alpha}(X)\left|\theta-\theta^{\prime}\right|^{\alpha}} .
\end{gathered}
$$

On the other hand, since

$$
c \frac{\left|\theta-\theta^{\prime}\right|^{\alpha}}{\theta^{\alpha}} \leq\left|h(\theta)-h\left(\theta^{\prime}\right)\right| \leq C \frac{\left|\theta-\theta^{\prime}\right|^{\alpha}}{\theta^{\alpha}} \text { if } X^{\prime} \in \widetilde{K}(X), \theta \text { near } 0,
$$

which means that in (8.2) the Hölder continuity in $\theta$ blows up like $\theta^{-\alpha}$ along the line $\theta=0$, and since $\widetilde{K}(X) \in K(X)$, we conclude that

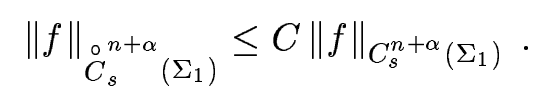

Definition 8.3 We denote by $C_{s}^{n+\alpha}\left(\mathbb{R}^{-}\right)$and $C_{s}^{n+\alpha}\left(\mathbb{R}^{+}\right)$the trace spaces of $C_{s}^{n+\alpha}(\Sigma)$ in each of the components of $\Gamma_{0}$ and $\Gamma_{1}$ respectively. The norm is identical to (8.1) but substituting $\Sigma$ by either one of the sets $\left(\mathbb{R}^{-}, \pm 1\right)=$ $\{(x, \pm 1) ;-\infty<x<0\}$ and $\left(\mathbb{R}^{+}, \pm 1\right)$ respectively.

Consider now the hyperbolic Problem $\left(\mathbf{H}_{0}\right)$ with $F=\epsilon\left(D_{0}+\widetilde{D}\right)$; its solution $T$ can be written in the form $T=U V$ where $U$ is a solution in $\Sigma$ of (3.1) established in Theorem 3.3 and $V$ is given by (3.2). In a neighborhood of $(0, \pm 1)$ we can write $T=T_{h}+T_{s}$ where $T_{h}$ and $T_{s}$ are given by (4.3), (4.4); recall that $T_{s}=I+J$ as in (4.5). In Sections 4-7 we estimated weighted Hölder norms of $I$ and $J$ (and thus of $T_{s}$ ) by a constant times the Hölder norms of $\widetilde{D}$, in a neighborhood of the points $(0, \pm 1)$. The same estimate holds for $T_{h}$ and the proof, in fact, is much simpler, for it uses Hölder estimates on $\int \frac{r^{\frac{1}{2}}(\eta)}{f(\eta)} d \eta$ already derived in Sections 4,5. Away from the points $(0, \pm 1)$ we can estimate the (nonweighted) Hölder norm of $T$ by obvious modifications, since the characteristics are uniformly $C^{1+\alpha}$ (cf. Th. 3.3).

Remark 8.2. We note that the assumption $g_{1}( \pm 1)=0$ implies compatibility of the boundary conditions for at $(-N, \pm 1)$, which enables us to conclude that $T$ is uniformly $C^{\alpha}$ in a neighborhood of these points. This assumption on $g_{1}$ and the fact that $g_{2}=$ const. near $y= \pm 1$ also imply compatibility 
conditions for the solution $\psi$ of (11.1)-(11.4) (in Section 11) which will allow us to deduce that $\psi$ is in $C^{2+\alpha}$ in a neighborhood of the four corner points $(-N, \pm 1),(N, \pm 1)$; this fact will be implicitly used in the future.

In view of Remarks 8.1 and 8.2 , we may conveniently recast the Hölder estimates derived above for $T$ in terms of the $\stackrel{\circ}{C}$ norm as follows:

Theorem 8.4 (i) The solution $\mathbf{T}$ of Problem $\left(\mathbf{H}_{0}\right)$ corresponding to $F=D_{0}+$ $\epsilon \widetilde{D}$ satisfies the estimate

$$
\|\mathbf{T}\|_{C}^{\circ \alpha\left(\Sigma_{i}\right)} \leq C\left(M+|\epsilon|\left(1+\|\widetilde{D}\|_{C}^{\circ \alpha\left(\Sigma_{i}\right)}\right)\right) \quad(i=1,2)
$$

where $M$ is a bound of the Hölder norm of $\left.T\right|_{x=-N}$.

(ii) If $\mathbf{T}_{1}$ is the solution of Problem $\left(\mathbf{H}_{0}\right)$ for $\widetilde{D}_{1}$ and $\mathbf{T}_{2}$ is the solution of problem $\left(\mathbf{H}_{0}\right)$ for $\widetilde{D}_{2}$, then

$$
\left\|\mathbf{T}_{1}-\mathbf{T}_{2}\right\|_{C}^{\circ \alpha}\left(\Sigma_{i}\right) \leq C|\epsilon|\left\|\widetilde{D}_{1}-\widetilde{D}_{2}\right\|_{C\left(\Sigma_{i}\right)}^{\circ \alpha} \quad(i=1,2) ;
$$

$C$ is a constant independent of $\epsilon$.

Here $\alpha$ is a and sufficiently small positive constant, depending on the parameters $a$ and $c$.

\section{Part III}

\section{Analysis of the elliptic problem}

\section{An elliptic problem in the half plane}

Let $\Sigma_{0}=\{(x, y) ;-\infty<x<\infty, 0<y<\infty\}$. In Section 8 we defined spaces $C_{s}^{n+\alpha}\left(\Sigma_{1}\right)$ and $\stackrel{\circ}{C}_{s}^{n+\alpha}\left(\Sigma_{1}\right)$; analogously, we define $C_{s}^{n+\alpha}\left(\Sigma_{0}\right)$ and $\stackrel{\circ}{C}_{s}^{n+\alpha}\left(\Sigma_{0}\right)$.

Set

$$
T=\left(\begin{array}{c}
T_{x x} \\
T_{y y} \\
T_{x y}
\end{array}\right)
$$

and define

$$
\|\mathbf{T}\|_{C_{s}^{n+\alpha}\left(\Sigma_{0}\right)}=\left\|T_{x x}\right\|_{C_{s}^{n+\alpha}\left(\Sigma_{0}\right)}+\left\|T_{y y}\right\|_{C_{s}^{n+\alpha}\left(\Sigma_{0}\right)}+\left\|T_{x y}\right\|_{C_{s}^{n+\alpha}\left(\Sigma_{0}\right)} \cdot
$$

In the same way we define $\|\mathbf{T}\|_{C_{s}{ }^{n+\alpha}\left(\Sigma_{0}\right)}$; the index $s$ will be usually dropped out if $s=0$.

In this and in the following section we study the auxiliary elliptic problem: Given $\mathbf{T} \in \stackrel{\circ}{C}_{0}^{\alpha}\left(\Sigma_{0}\right)$ solve for $u$,

$$
\begin{aligned}
\Delta^{2} u & =\Delta T_{x y}-2 \partial_{x x}^{2} T_{x y}+\partial_{x y}^{2}\left(T_{x x}-T_{y y}\right) \text { in } \Sigma_{0} \\
u & =0 \text { at } y=0 \\
u_{, y} & =0 \text { at } y=0, x<0 \\
u_{, y y} & =0 \text { at } y=0, x>0 .
\end{aligned}
$$


Here the second order derivatives of $T$ are taken in the distribution sense.

We shall prove the following theorem:

Theorem 9.1 For any $\mathbf{T} \in \stackrel{\circ}{C}_{0}^{\alpha}\left(\Sigma_{0}\right)$, there exists a unique (weak) solution $u$ of problem (9.1)-(9.4) in $\stackrel{\circ}{C}_{2}^{2+\alpha}\left(\Sigma_{0}\right)$ and

$$
\|u\|_{C_{2}^{\circ 2+\alpha}\left(\Sigma_{0}\right)} \leq C\|\mathbf{T}\|_{{\stackrel{\circ}{C}\left(\Sigma_{0}\right)}^{\alpha}}
$$

where $C$ is a constant independent of $\mathbf{T}$.

To prove existence we decompose $u$ into $u=u_{1}+u_{2}$ where $u_{1}$ is a solution of the problem

$$
\begin{aligned}
\Delta^{2} u_{1} & =\Delta T_{x y}-2 \partial_{x x}^{2} T_{x y}+\partial_{x y}^{2}\left(T_{x x}-T_{y y}\right) \equiv F \text { in } \Sigma_{0} \\
u_{1} & =0 \quad \text { at } y=0, x>0 \\
u_{1, y y} & =0 \quad \text { at } y=0, x>0
\end{aligned}
$$

( $u_{1}$ is not unique), and $u_{2}$ is a solution of

$$
\begin{aligned}
\Delta^{2} u_{2} & =0 \text { in } \Sigma_{0}, \\
u_{2} & =0 \text { at } y=0, x>0, \\
u_{2} & =-u_{1} \text { at } y=0, x<0, \\
u_{2, y} & =-u_{1, y} \text { at } y=0, x<0, \\
u_{2, y y} & =0 \text { at } y=0, x>0 .
\end{aligned}
$$

We further decompose the problem (9.5)-(9.7) into the two problems:

$$
\left\{\begin{array}{c}
\Delta \Phi_{1}=F \text { in } \Sigma_{0}, \\
\Phi_{1}=0 \text { at } y=0, x>0
\end{array}\right.
$$

and

$$
\left\{\begin{array}{c}
\Delta u_{1}=\Phi \text { in } \Sigma_{0}, \\
u_{1}=0 \text { at } y=0, x>0 .
\end{array}\right.
$$

From the form of $F$ it follows that we can write $u_{1}=u_{11}+u_{12}$ and $\Phi_{1}=$ $\Phi_{11}+\Phi_{12}$ where

$$
\begin{gathered}
\left\{\begin{array}{c}
\Delta \Phi_{11}=\Delta T_{x y} \text { in } \Sigma_{0}, \\
\Phi_{11}=0 \text { at } y=0, x>0,
\end{array}\right. \\
\left\{\begin{array}{c}
\Delta u_{11}=\Phi_{11} \text { in } \Sigma_{0}, \\
u=0 \text { at } y=0, x>0,
\end{array}\right.
\end{gathered}
$$

and

$$
\begin{aligned}
& \left\{\begin{array}{c}
\Delta \Phi_{12}=-2 \partial_{x x}^{2} T_{x y}+\partial_{x y}^{2}\left(T_{x x}-T_{y y}\right) \text { in } \Sigma_{0}, \\
\Phi_{12}=0 \text { at } y=0, x>0,
\end{array}\right. \\
& \left\{\begin{array}{c}
\Delta u_{12}=\Phi_{12} \text { in } \Sigma_{0} \\
u_{12}=0 \text { at } y=0, x>0 .
\end{array}\right.
\end{aligned}
$$


The solution of (9.13) has the form $\Phi_{11}=T_{x y}+\varphi$, where

$$
\left\{\begin{array}{c}
\Delta \varphi=0 \text { in } \Sigma_{0}, \\
\varphi=-T_{x y} \text { at } y=0, x>0 .
\end{array}\right.
$$

In Section 10 we prove the following lemmas:

Lemma 9.2 There exists a solution $\varphi$ of Problem (9.17) such that

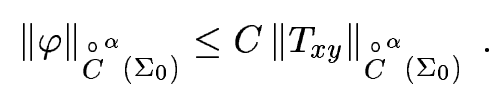

Lemma 9.3 There exists a solution $\Phi_{12}$ of Problem (9.15) such that

$$
\left\|\Phi_{12}\right\|_{C\left(\Sigma_{0}\right)}^{\circ \alpha} \leq C\|\mathbf{T}\|_{C\left(\Sigma_{0}\right)} .
$$

Lemma 9.4 There exists a solution $u_{11}$ of Problem (9.14) such that

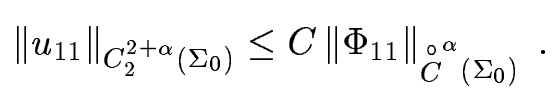

Note that $\left\|\Phi_{11}\right\|_{C\left(\Sigma_{0}\right)}^{\circ \alpha} \leq C\left\|T_{x y}\right\|_{C\left(\Sigma_{0}\right)}^{\circ \alpha}$, by Lemma 9.3 , so that

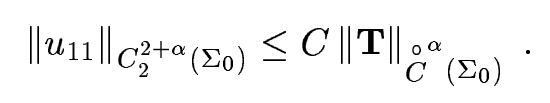

Lemma 9.4 applies also to problem (9.16) and therefore we have:

Corollary 9.5 There exists a solution $u_{12}$ of problem (9.16) such that

$$
\left\|u_{12}\right\|_{C_{2}^{2+\alpha}\left(\Sigma_{0}\right)} \leq C\left\|\Phi_{12}\right\|_{C}^{\circ \alpha}\left(\Sigma_{0}\right)
$$

and, consequently, by Lemma 9.3,

$$
\left\|u_{12}\right\|_{C_{2}^{2+\alpha}\left(\Sigma_{0}\right)} \leq C\|\mathbf{T}\|_{\stackrel{\rho}{\alpha}^{\alpha}\left(\Sigma_{0}\right)}
$$

From (9.20) and (9.22) we get:

\section{Corollary 9.6}

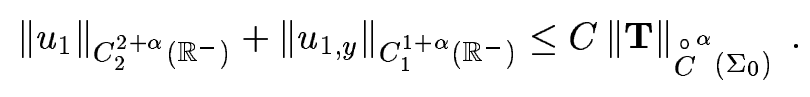

We shall use the estimate (9.23) in order to solve problem (9.8)-(9.12). The system (9.8)-(9.12) consists of a biharmonic equation

$$
\Delta^{2} u_{2}=0 \text { in } \Sigma_{0}
$$

in the half plane with mixed boundary conditions

$$
\begin{array}{r}
u_{2}=b_{1}(x) \text { at } y=0, x<0, \\
u_{2}=b_{2}(x) \text { at } y=0, x>0, \\
u_{2, y}=b_{3}(x) \text { at } y=0, x<0, \\
u_{2, y y}=b_{4}(x) \text { at } y=0, x>0 .
\end{array}
$$


By a general theory developed in [6], [7], [9], [10] and the references therein, it follows that in the special case of the system (9.24)-(9.28) the following holds: Suppose that $b_{1}, b_{2} \in C_{2}^{2+\alpha}, b_{3} \in C_{1}^{1+\alpha}, b_{4} \in C^{\alpha}$ and there exists a positive constant $\gamma$ such that $|\lambda-2|>\gamma$ for any nontrivial solution

$$
u=r^{\lambda} f(\theta), \lambda \in \mathbb{C}
$$

to the homogeneous system associated to (9.24)-(9.28). Then there exists a unique solution of system (9.24)-(9.28) such that

$$
\left\|u_{2}\right\|_{C_{2}^{2+\alpha}\left(\Sigma_{0}\right)} \leq C\left(\left\|b_{1}, b_{2}\right\|_{C_{2}^{2+\alpha}}+\left\|b_{3}\right\|_{C_{1}^{1+\alpha}}+\left\|b_{4}\right\|_{C_{0}^{\alpha}}\right) .
$$

To check the last condition, we substitute (9.29) into (9.24). We find that $f(\theta)$ has to satisfy

$$
f^{(i v)}+\left(\lambda^{2}+(\lambda-2)^{2}\right) f^{\prime \prime}+\lambda^{2}(\lambda-2)^{2} f=0
$$

so that

$f(\theta)=A \sin (\lambda \theta)+B \cos (\lambda \theta)+C \sin ((\lambda-2) \theta)+D \cos ((\lambda-2) \theta)$ if $\lambda \neq 0,1,2$,

$f(\theta)=A+B \theta+C \sin (2 \theta)+D \cos (2 \theta)$ if $\lambda=0$ or $\lambda=2$,

$f(\theta)=A \sin \theta+B \cos \theta+C \theta \sin \theta+D \theta \cos \theta$ if $\lambda=1$.

Imposing the homogeneous boundary conditions we get, for $\lambda \neq 0,1,2$, the system

$$
\begin{aligned}
B+D & =0, \\
\lambda A+(\lambda-2) C & =0, \\
(A+C) \sin (\lambda \pi)+(B+D) \cos (\lambda \pi) & =0 \\
-\left(\lambda^{2} A+(\lambda-2)^{2} C\right) \sin (\lambda \pi)-\left(\lambda^{2} B+(\lambda-2)^{2} D\right) \cos (\lambda \pi) & =0 .
\end{aligned}
$$

The system has a solution only if the determinant of the coefficients is zero, i.e., if $4(\lambda-1) \sin (2 \lambda \pi)=0$. This yields $\lambda_{n}=\frac{n}{2}, n \in \mathbb{Z}$. If $\lambda=0,2$ then we obtain from the boundary conditions the system

$$
A+D=0, B+2 C=0, A+B \pi+D=0,-2 D=0
$$

which has no nontrivial solutions. Finally, if $\lambda=1$, we have the system

$$
B=0, A+D=0,-B+D \pi=0,-B-2 C-\pi D=0
$$

which again has no nontrivial solutions. Therefore, the only admissible exponents are $\left\{\lambda_{n}\right\}=\left\{\frac{3}{2} \cup\left\{\frac{n}{2}\right\}_{n=5}^{\infty}\right\}$, so that $\left|\lambda_{n}-2\right| \geq \gamma>0$.

In view of (9.23), we can now apply the result of [6], [7], [9], [10] to conclude that there exists a solution $u_{2}$ of (9.24)-(9.28) satisfying

$$
\left\|u_{2}\right\|_{C_{2}^{2+\alpha}\left(\Sigma_{0}\right)} \leq C\|\mathbf{T}\|_{{\stackrel{\rho}{O}\left(\Sigma_{0}\right)}^{\alpha}} .
$$

Recalling also (9.20) and (9.22), the existence proof of Theorem 9.1 is complete. Uniqueness in space $C_{2}^{2+\alpha}$ follows immediately from the general theory of [9]. 


\section{Proofs of Lemmas 9.2-9.4}

\section{Proof of Lemma 9.2}

By the change of variables

$$
\sqrt{x^{2}+y^{2}}=r=e^{t}, \arctan \frac{y}{x}=\theta, \varphi(x, y)=\psi(t, \theta), T_{x y}(x .0)=S(t)(x>0)
$$

we find that

$$
\Delta \varphi=e^{-2 t}\left(\frac{\partial^{2} \varphi}{\partial t^{2}}+\frac{\partial^{2} \varphi}{\partial \theta^{2}}\right) \equiv e^{-2 t} \Delta_{t \theta} \psi
$$

so that

$$
\begin{aligned}
\Delta \psi & =0 \text { in } \Omega=\{-\infty<t<\infty, 0<\theta<2 \pi\}, \\
\psi(t, 0) & =-S(t),-\infty<t<\infty .
\end{aligned}
$$

We can represent one solution by means of Poisson's kernel

$$
\psi(t, \theta)=-\frac{1}{\pi} \int_{-\infty}^{\infty} \frac{\theta}{(t-\xi)^{2}+\theta^{2}} S(\xi) d \xi
$$

Since the weighted Hölder norms in the $(x, y)$ variable are equivalent to the nonweighted Hölder norms in the $(\log r, \theta)$ variable (see Remark 8.1), the estimate (9.18), when written in the $(t, \theta)$ variables, means that

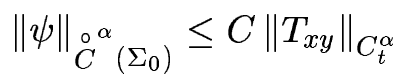

where

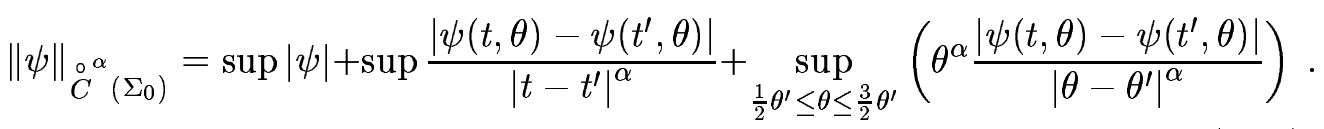

Clearly,

$$
\sup |\psi| \leq \frac{1}{\pi} \int_{-\infty}^{+\infty} \frac{\theta}{(t-\xi)^{2}+\theta^{2}} \sup \left|T_{x y}\right| d \xi \leq \sup \left|T_{x y}\right|
$$

Next we have

$$
\begin{gathered}
\psi(t+h, \theta)-\psi(t, \theta) \\
=-\frac{1}{\pi} \int_{-\infty}^{+\infty} \frac{\theta}{(t+h-\xi)^{2}+\theta^{2}} T_{x y}(\xi, 0) d \xi+\frac{1}{\pi} \int_{-\infty}^{+\infty} \frac{\theta}{(t-\xi)^{2}+\theta^{2}} T_{x y}(\xi, 0) d \xi \\
=-\frac{1}{\pi} \int_{-\infty}^{+\infty} \frac{\theta}{(t-\xi)^{2}+\theta^{2}}\left(T_{x y}(\xi-h, 0)-T_{x y}(\xi, 0)\right) d \xi
\end{gathered}
$$

and then

$$
\begin{gathered}
|\psi(t+h, \theta)-\psi(t, \theta)| \leq\left[\sup \frac{\left|T_{x y}(\xi-h, 0)-T_{x y}(\xi, 0)\right|}{h^{\alpha}}\right] \frac{h^{\alpha}}{\pi} \int_{-\infty}^{+\infty} \frac{\theta}{(t-\xi)^{2}+\theta^{2}} d \xi \\
\leq C\left\|T_{x y}\right\|_{C_{t}^{\alpha}} h^{\alpha} .
\end{gathered}
$$


Consider finally,

$$
\begin{gathered}
\psi(t, \theta+h)-\psi(t, \theta) \\
=-\frac{1}{\pi} \int_{-\infty}^{+\infty} \frac{\theta+h}{(t-\xi)^{2}+(\theta+h)^{2}} T_{x y}(\xi, 0) d \xi+\frac{1}{\pi} \int_{-\infty}^{+\infty} \frac{\theta}{(t-\xi)^{2}+\theta^{2}} T_{x y}(\xi, 0) d \xi
\end{gathered}
$$

where $\frac{\theta}{2}<h<\theta$. By the mean value theorem,

$$
|\psi(t, \theta+h)-\psi(t, \theta)| \leq \frac{1}{\pi} h \int_{-\infty}^{+\infty} \frac{\left|(t-\xi)^{2}-\left(\theta+h^{*}\right)^{2}\right|}{\left[(t-\xi)^{2}+\left(\theta+h^{*}\right)^{2}\right]^{2}}\left|T_{x y}(\xi, 0)\right| d \xi
$$

for some $h^{*} \in(0, h)$; note that $h<\left(\theta+h^{*}\right)<3 h$. We can then estimate

$$
\begin{aligned}
|\psi(t, \theta+h)-\psi(t, \theta)| & \leq \frac{1}{\pi} h \sup \left|T_{x y}\right| \frac{1}{\left(\theta+h^{*}\right)} \int_{-\infty}^{+\infty} \frac{\left|1-x^{2}\right|}{\left(1+x^{2}\right)^{2}} d x \\
& \leq C \frac{h^{\alpha}}{\theta^{\alpha}}\left(\frac{h^{1-\alpha}}{\left(\theta+h^{*}\right)^{1-\alpha}}\right) \sup \left|T_{x y}\right| \leq C \frac{h^{\alpha}}{\theta^{\alpha}} \sup \left|T_{x y}\right|
\end{aligned}
$$

\section{Proof of Lemma 9.3}

By change of variables

$$
r=e^{t}, \Phi_{12}(x, y)=v(t, \theta)
$$

we have, as before,

$$
\Delta \Phi_{12}=e^{-2 t}\left(\frac{\partial^{2} v}{\partial t^{2}}+\frac{\partial^{2} v}{\partial \theta^{2}}\right) \equiv e^{-2 t} \Delta_{t \theta} v
$$

and the right-hand side of the Poisson equation (9.15) becomes a linear combination of $e^{-2 t}$ times

$$
\partial_{t t}^{2} T, \partial_{t \theta}^{2} T,(\sin \theta) \partial_{\theta \theta}^{2} T, \partial_{t} T, \partial_{\theta} T
$$

with smooth bounded coefficients, where $T$ stands for any of the components $T_{x x}, T_{x y}, T_{y y}$. Thus it will suffice to solve an equation of the form

$$
\begin{aligned}
\Delta v & =g S \text { in } \Omega=\{-\infty<t<\infty, 0<\theta<\pi\}, \\
v & =0 \text { at } \theta=0
\end{aligned}
$$

where $S$ is one of the functions in (10.6) and $g$ is a smooth bounded function.

We represent $v$ by means of Green's function in the half-space $\Omega$ :

$$
v=\int_{\Omega} G(t-\xi, \theta-\eta) g(\xi, \eta) S(\xi, \eta) d \xi d \eta
$$

where

$$
G(t-\xi, \theta, \eta)=\frac{1}{2 \pi}\left(\log \left[(t-\xi)^{2}+(\theta-\eta)^{2}\right]-\log \left[(t-\xi)^{2}+(\theta+\eta)^{2}\right]\right)
$$

the integral in (10.7) is taken in the principal value sense. 
Assume first that $g \equiv 1$. We begin with the case $S=\partial_{t \theta}^{2} \widehat{T}$ where $\widehat{T}(t, \theta)=$ $T(x, y)$. We can write $v$ in the form

$$
v(t, \theta)=\int_{\Omega} G(t-\xi, \theta, \eta) \partial_{\xi \eta}^{2}(\widehat{T}(\xi, \eta)-\widehat{T}(t, \theta)) d \xi d \eta
$$

Take a ball $B_{R_{0}}\left(t^{0}, \theta^{0}\right)$ such that $B_{\frac{R_{0}}{2}}\left(t^{0}, \theta^{0}\right)$ contains a neighborhood of $(t, \theta)$. We can break up the integral in (10.8) into

$$
\int_{\Omega \cap B_{R_{0}}\left(t^{0}, \theta^{0}\right)}+\int_{\Omega \backslash B_{R_{0}}\left(t^{0}, \theta^{0}\right)}=v_{1}(t, \theta)+v_{2}(t, \theta) .
$$

Since $v_{2}(t, \theta)$ is clearly uniformly smooth in $\bar{\Omega}$-neighborhood of $\left(t^{0}, \theta^{0}\right)$ and it satisfies the asserted estimates of the lemma, it suffices to consider $v_{1}(t, \theta)$.

Integration by parts with respect to $\eta$ yields

$$
\begin{aligned}
v_{1}(t, \theta) & =-\int_{\Omega \cap B_{R_{0}}\left(t^{0}, \theta^{0}\right)} N(t-\xi, \theta, \eta) \partial_{\xi}(\widehat{T}(\xi, \eta)-\widehat{T}(t, \theta)) d \xi d \eta+J(t, \theta) \\
& \equiv v_{3}(t, \theta)+J(t, \theta)
\end{aligned}
$$

where

$$
N(t-\xi, \theta, \eta) \equiv \partial_{\eta} G(t-\xi, \theta, \eta)
$$

and $J(t, \theta)$ is a boundary integral over the part of $\Omega \cap \partial B_{R_{0}}\left(t^{0}, \theta^{0}\right)$ which does not lie on the line $\eta=0$; we used here the fact that

$$
G(t-\xi, 0, \eta)=0 \text { at } \eta=0 .
$$

Since the boundary integral is uniformly smooth, it remains to consider $v_{3}$. Note that in the expression for $v_{3}$ we may replace $\widehat{T}(t, \theta)$ by $\widehat{T}(t, \eta)$. Then, integration by parts in $\xi$ yields

$$
\begin{aligned}
v_{3}(t, \theta) & =\int_{\Omega \cap B_{R_{0}}\left(t^{0}, \theta^{0}\right)} \partial_{\xi} N(t-\xi, \theta, \eta)(\widehat{T}(\xi, \eta)-\widehat{T}(t, \eta)) d \xi d \eta+J_{1}(t, \theta) \\
& \equiv v_{4}(t, \theta)+J_{1}(t, \theta) .
\end{aligned}
$$

Once again the boundary integral is taken over the boundary of $\Omega \cap \partial B_{R_{0}}\left(t^{0}, \theta^{0}\right)$ which does not lie in $\eta=0$, and it remains to consider $v_{4}(t, \theta)$.

But $v_{4}$ has a form for which the proof of the Schauder estimates ([4], p. 57) yields:

$$
\sup _{B_{\frac{R_{0}}{2}}\left(t^{0}, \theta^{0}\right)}\left|v_{4}\right|+\sup _{(t, \theta),\left(t^{\prime}, \theta\right) \in B_{\frac{R_{0}}{2}}\left(t^{0}, \theta^{0}\right)} \frac{\left|v_{4}(t, \theta)-v_{4}\left(t^{\prime}, \theta\right)\right|}{\left|t-t^{\prime}\right|^{\alpha}} \leq C\|\widehat{T}\|_{C}^{\circ \alpha}{ }_{\left.\frac{\circ}{2} B_{\frac{R_{0}}{2}}\left(t^{0}, \theta^{0}\right)\right)} .
$$

Since, on the right hand side, we only use the Hölder coefficient of $\widehat{T}(\xi, \eta)$ in $\xi$, we conclude that

$$
\sup |v|+\sup _{t^{\prime} \in B_{\frac{t}{2}}(t, \theta)} \frac{\left|v(t, \theta)-v\left(t^{\prime}, \theta\right)\right|}{\left|t-t^{\prime}\right|^{\alpha}} \leq C\|\widehat{T}\|_{C\left(\Sigma_{0}\right)} .
$$


Next we repeat the above process, but integrating by parts (in $v_{1}$ ) first in $\xi$, to obtain

$$
\begin{aligned}
v_{1}(t, \theta)= & -\int_{\Omega \cap B_{R_{0}}\left(t^{0}, \theta^{0}\right)} \partial_{\xi} G(t-\xi, \theta, \eta) \partial_{\eta}(\widehat{T}(\xi, \eta)-\widehat{T}(t, \theta)) d \xi d \eta \\
& + \text { boundary integrals as before },
\end{aligned}
$$

and we may replace in the area integral which we shall denote by $v_{5}(t, \theta), \widehat{T}(t, \theta)$ by $\widehat{T}(\xi, \theta)$. Then by integration by parts (using (10.9)),

$$
\begin{aligned}
v_{5}(t, \theta) & =\int_{\Omega \cap B_{R_{0}}\left(t^{0}, \theta^{0}\right)} \partial_{\xi \eta}^{2} G(t-\xi, \theta, \eta)(\widehat{T}(\xi, \eta)-\widehat{T}(\xi, \theta)) d \xi d \eta+J_{3}(t, \theta) \\
& \equiv v_{6}(t, \theta)+J_{3}(t, \theta)
\end{aligned}
$$

where $J_{3}$ is a boundary integral taken over the part of $\partial\left(\Omega \cap B_{R_{0}}\left(t^{0}, \theta^{0}\right)\right)$ which does not lie on $\eta=0$.

The proof of the interior Schauder estimates (i.e., with weighted distance to the boundary $\theta=0$ ) then gives ([4], p. 62)

$$
\sup _{\left(t, \theta^{\prime}\right) \in B_{\frac{\theta}{2}}(t, \theta)} \theta^{\alpha} \frac{\left|v_{6}(t, \theta)-v_{6}\left(t, \theta^{\prime}\right)\right|}{\left|\theta-\theta^{\prime}\right|^{\alpha}} \leq C\|\widehat{T}\|_{C}^{\circ \alpha}{ }_{\left(B_{R_{0}}\left(t^{0}, \theta^{0}\right)\right)} .
$$

uniformly for $(t, \theta) \in B_{\frac{R_{0}}{2}}\left(t^{0}, \theta^{0}\right)$ (here we use the weighted Hölder continuity of $\widehat{T}(\xi, \eta)$ in $\eta)$. Hence

$$
\sup _{\left(t, \theta^{\prime}\right) \in B_{\frac{\theta}{2}}(t, \theta)} \theta^{\alpha} \frac{\left|v(t, \theta)-v\left(t, \theta^{\prime}\right)\right|}{\left|\theta-\theta^{\prime}\right|^{\alpha}} \leq C\|\widehat{T}\|_{C\left(\Sigma_{0}\right)}^{\circ \alpha} .
$$

Combining (10.10) with (10.11), the lemma follows for $\partial_{\xi \eta}^{2} \widehat{T}$.

In case $S=\partial_{\xi \xi}^{2} \widehat{T}$ we end up with the integral

$$
v_{7}(t, \theta)=\int_{\Omega \cap B_{R_{0}}\left(t^{0}, \theta^{0}\right)} \partial_{\xi \xi}^{2} G(t-\xi, \theta, \eta)(\widehat{T}(\xi, \eta)-\widehat{T}(t, \eta)) d \xi d \eta
$$

for which we have a bound similar to (10.10).

To obtain a bound similar to (10.11) we need to use the fact

$$
\partial_{\xi \xi}^{2} G(t-\xi, \theta, \eta)=0 \text { at } \eta=0
$$

or, more precisely, that

$$
\begin{aligned}
\partial_{\xi \xi}^{2} G(t-\xi, \theta, \eta) & =-\frac{4}{\pi} \theta \eta \frac{\partial}{\partial \xi}\left[\frac{(t-\xi)}{\left[(t-\xi)^{2}+(\theta-\eta)^{2}\right]\left[(t-\xi)^{2}+(\theta+\eta)^{2}\right]}\right] \\
& \equiv \theta \eta K(t-\xi, \theta, \eta) .
\end{aligned}
$$

We have then

$$
v_{7}(t, \theta)=\int_{\Omega \cap B_{R_{0}}\left(t^{0}, \theta^{0}\right)} \partial_{\xi \xi}^{2} G(t-\xi, \theta, \eta)(\widehat{T}(\xi, \eta)-\widehat{T}(t, \theta)) d \xi d \eta
$$




$$
\begin{aligned}
& =\int_{\Omega \cap B_{R_{0}}\left(t^{0}, \theta^{0}\right)} \partial_{\xi \xi}^{2} G(t-\xi, \theta, \eta)[(\widehat{T}(\xi, \eta)-\widehat{T}(t, \eta))+(\widehat{T}(t, \eta)-\widehat{T}(t, \theta))] d \xi d \eta \\
& \equiv v_{7,1}+v_{7,2}
\end{aligned}
$$

The derivation of the Hölder estimate for $v_{7,2}$ is identical to that for the case $S=\partial_{\xi \eta}^{2} \widehat{T}$; it uses the interior Schauder estimates in [4], p. 62. The estimate for $v_{7,1}$ uses formula (10.13). Accordingly, it is possible to obtain, for every $\left(t, \theta^{\prime}\right) \in B_{\frac{\theta}{2}}(t, \theta)$

$$
\begin{aligned}
& \left|v_{7,1}(t, \theta)-v_{7,1}\left(t, \theta^{\prime}\right)\right| \\
\leq & C\|\widehat{T}\|_{C\left(\Sigma_{0}\right)} \int_{\Omega \cap B_{R_{0}}\left(t^{0}, \theta^{0}\right)}\left|\theta \eta K(t-\xi, \theta, \eta)-\theta^{\prime} \eta K\left(t-\xi, \theta^{\prime}, \eta\right)\right||t-\xi|^{\alpha} d \xi d \eta \\
\leq & C\|\widehat{T}\|_{{\stackrel{\circ}{\alpha}\left(\Sigma_{0}\right)}^{\alpha}}\left|\theta-\theta^{\prime}\right| \int_{\Omega \cap B_{R_{0}}\left(t^{0}, \theta^{0}\right)}|\eta K(t-\xi, \theta, \eta)||t-\xi|^{\alpha} d \xi d \eta \\
& +C\|\widehat{T}\|_{C\left(\Sigma_{0}\right)} \theta \int_{\Omega \cap B_{R_{0}}\left(t^{0}, \theta^{0}\right)}\left|\eta\left(K(t-\xi, \theta, \eta)-K\left(t-\xi, \theta^{\prime}, \eta\right)\right)\right||t-\xi|^{\alpha} d \xi d \eta \\
\equiv & I_{1}+I_{2} .
\end{aligned}
$$

It is easy to verify that

$$
\begin{aligned}
\int_{\Omega \cap B_{R_{0}}\left(t^{0}, \theta^{0}\right)}|\eta K(t-\xi, \theta, \eta)||t-\xi|^{\alpha} d \xi d \eta & \leq \frac{C}{\theta} \\
\int_{\Omega \cap B_{R_{0}}\left(t^{0}, \theta^{0}\right)}\left|\eta\left(K(t-\xi, \theta, \eta)-K\left(t-\xi, \theta^{\prime}, \eta\right)\right)\right||t-\xi|^{\alpha} d \xi d \eta & \leq C \frac{\left|\theta-\theta^{\prime}\right|}{\theta \theta^{\prime}}
\end{aligned}
$$

and, if we use the fact that $\left(t, \theta^{\prime}\right) \in B_{\frac{\theta}{2}}(t, \theta)$, then we get

$$
\begin{aligned}
& I_{1} \leq C\|\widehat{T}\|_{C^{\circ}\left(\Sigma_{0}\right)} \frac{\left|\theta-\theta^{\prime}\right|}{\theta} \leq C\|\widehat{T}\|_{C^{\circ}\left(\Sigma_{0}\right)} \frac{\left|\theta-\theta^{\prime}\right|^{\alpha}}{\theta^{\alpha}}, \\
& I_{2} \leq C\|\widehat{T}\|_{C^{\circ}\left(\Sigma_{0}\right)} \frac{\left|\theta-\theta^{\prime}\right|}{\theta^{\prime}} \leq C\|\widehat{T}\|_{C^{\circ}\left(\Sigma_{0}\right)} \frac{\left|\theta-\theta^{\prime}\right|^{\alpha}}{\theta^{\alpha}} .
\end{aligned}
$$

Hence

$$
\sup _{\left(t, \theta^{\prime}\right) \in B_{\frac{\theta}{2}}(t, \theta)} \theta^{\alpha} \frac{\left|v_{7,1}(t, \theta)-v_{7,1}\left(t, \theta^{\prime}\right)\right|}{\left|\theta-\theta^{\prime}\right|^{\alpha}} \leq C\|\widehat{T}\|_{C}{ }_{\circ\left(\Sigma_{0}\right)}
$$

Finally, if $S=\sin \eta \partial_{\eta \eta}^{2} \widehat{T}$ we can still integrate by parts twice in $\eta$, and use the fact that $\sin \eta \sim \eta$ when $\eta \rightarrow 0$ to derive, as before, estimates of the type (10.10) and (10.11). So far we have assumed that $g \equiv 1$. If $g \neq 1$ then we simply replace $G$ by $g G$ in the above arguments.

Collecting all these results, the lemma follows.

Proof of Lemma 9.4

The proof of Lemma 9.4 is very similar to the proof of Lemma 9.3. In terms of $(x, y)$ coordinates, Problem (9.16) reads (omitting subindexes)

$$
\left\{\begin{array}{c}
\Delta u=\Phi \text { in } \Sigma_{0} \\
u=0 \text { at } y=0, x>0
\end{array}\right.
$$


If we apply the operator $\partial_{x}^{2}$ to system (10.14), then we get a system identical in structure to (9.15) so that, by Lemma 9.3 ,

$$
\left\|\partial_{x}^{2} u\right\|_{C\left(\Sigma_{0}\right)}^{\circ \alpha} \leq C\|\Phi\|_{C}^{\circ \alpha}\left(\Sigma_{0}\right)
$$

where $\partial_{x}^{2} u$ is given (at leading order) by (10.12) with $\widehat{T}$ substituted by $\Phi$.

From equation (10.14) we get

$$
\partial_{y}^{2} u=-\partial_{x}^{2} u+\Phi
$$

and therefore

$$
\left\|\partial_{y}^{2} u\right\|_{C\left(\Sigma_{0}^{\alpha}\right)} \leq C\left(\left\|\partial_{x}^{2} u\right\|_{{\stackrel{\circ}{\circ}\left(\Sigma_{0}\right)}^{\alpha}}+\|\Phi\|_{{\stackrel{\circ}{\alpha}\left(\Sigma_{0}\right)}^{\alpha}}\right) \leq C\|\Phi\|_{C\left(\Sigma_{0}^{\alpha}\right)} .
$$

Thus it only remains to estimate $\partial_{x y}^{2} u$. Since its leading order term is given by (10.12) with $\partial_{\xi \xi}^{2} G(t-\xi, \theta-\eta)$ substituted by $\partial_{\eta \xi}^{2} G(t-\xi, \theta-\eta)$, the estimates of the corresponding integrals then follow along the same lines as in the previous lemma.

To estimate the lower order derivatives of $u$ we write

$$
u_{r}=\int_{0}^{r} u_{r r} d \rho, \quad u=\int_{0}^{r} d \rho \int_{0}^{\rho} u_{r r} d \rho^{\prime}, \quad u_{\theta}=\int_{0}^{r} u_{r \theta} d \rho .
$$

Then, clearly,

$$
\begin{aligned}
\left|u_{r}\right| & \leq r \sup \left|u_{r r}\right| \\
|u| & \leq r^{2} \sup \left|u_{r r}\right|, \\
\left|\frac{1}{r} u_{\theta}\right| & \leq r \sup \left|\frac{1}{r} u_{r \theta}\right| .
\end{aligned}
$$

Together with the estimates derived for $D^{2} u$ we then get

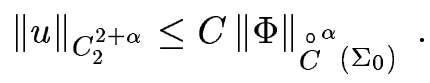

\section{The complete problem}

In this section we will solve the following problem:

$$
\begin{aligned}
\Delta^{2} \psi & =F[\widetilde{\psi}], \\
\psi & =0 \text { at } y= \pm 1, \\
\psi_{y} & =0 \text { at }-N<x<0, y= \pm 1, \\
\psi_{y y} & =G[\widetilde{\psi}] \text { at } 0<x<N, y= \pm 1 \\
\psi & =\psi_{y}=0 \text { at }|x|=N,|y|<1
\end{aligned}
$$

$F[\widetilde{\psi}]$ is an integrodifferential operator on $\widetilde{\psi}$ defined by

$$
\begin{aligned}
F[\widetilde{\psi}]= & -\Delta^{2} \psi_{0}+\frac{\alpha_{1}}{\alpha_{2}} \operatorname{Re} \operatorname{curl}[(\overrightarrow{\vec{v}} \cdot \nabla) \overrightarrow{\vec{v}}]+\frac{\epsilon}{\epsilon-1} \operatorname{curl} \operatorname{div}\left(\mathbf{K}^{-1} D_{0}\right) \\
& +\frac{\epsilon}{\epsilon-1} \operatorname{curl} \operatorname{div}\left(\mathbf{K}^{-1} \widetilde{D}\right)
\end{aligned}
$$


where

$$
\begin{gathered}
\widetilde{\vec{v}}=\left(\widetilde{\psi_{y}},-\widetilde{\psi}_{x}\right), \\
\widetilde{D}=\left(\begin{array}{cc}
\frac{\partial \widetilde{v_{x}}}{\partial x} & \frac{1}{2}\left(\frac{\partial \widetilde{v_{x}}}{\partial y}+\frac{\partial \widetilde{v_{y}}}{\partial x}\right) \\
\frac{1}{2}\left(\frac{\partial \widetilde{v_{x}}}{\partial y}+\frac{\partial \widetilde{v_{y}}}{\partial x}\right) & \frac{\partial y}{\partial y}
\end{array}\right),
\end{gathered}
$$

and $G[\widetilde{\psi}]$ is defined by

$$
G[\widetilde{\psi}]=\frac{\epsilon}{\epsilon-1} T_{x y}=\frac{\epsilon}{\epsilon-1}\left(\mathbf{K}^{-1}\left(D_{0}+\widetilde{D}\right)\right)_{x y} \quad \text { on } y= \pm 1,0<x<N .
$$

We can decompose $\psi$ as follows

$$
\psi=\psi_{0}+\psi_{1}+\psi_{2}+\psi_{3}+\psi_{4}
$$

where

$$
\begin{gathered}
\Delta^{2} \psi_{1}=-\Delta^{2} \psi_{0}+\frac{\alpha_{1}}{\alpha_{2}} \operatorname{Re} \operatorname{curl}[(\overrightarrow{\vec{v}} \cdot \nabla) \overrightarrow{\vec{v}}] \equiv \frac{\alpha_{1}}{\alpha_{2}} \operatorname{Re} f_{1}(x, y) \text { in } \Sigma, \\
\Delta^{2} \psi_{2}=\frac{\epsilon}{\epsilon-1} \operatorname{curl} \operatorname{div}\left(\mathbf{K}^{-1} D_{0}\right) \equiv \epsilon f_{2}(x, y) \text { in } \Sigma \\
\Delta^{2} \psi_{3}=\frac{\epsilon}{\epsilon-1} \operatorname{curl} \operatorname{div}\left(\mathbf{K}^{-1} \widetilde{D}\right) \equiv \epsilon f_{3}(x, y) \text { in } \Sigma \\
\Delta^{2} \psi_{4}=0
\end{gathered}
$$

and the functions $\psi_{i}$, for $i=1,2,3$, satisfy the boundary conditions:

$$
\begin{aligned}
\psi_{i}(x, \pm 1) & =0 \text { at }|x| \leq N \\
\psi_{i, y}(x, \pm 1) & =0 \text { at }-N<x<0 \\
\psi_{i, y y}(x, \pm 1) & =0 \text { at } 0<x<N \\
\psi_{i}( \pm 1, y) & =\psi_{i, y}( \pm 1, y)=0 \text { at }|x|=N
\end{aligned}
$$

while $\psi_{4}$ satisfies the boundary conditions (11.10), (11.11), (11.13) together with

$$
\psi_{i, y y}(x, \pm 1)=\frac{\epsilon}{\epsilon-1}\left(\mathbf{K}^{-1}\left(D_{0}+\widetilde{D}\right)\right)_{x y} \quad \text { at } 0<x<N
$$

Note that $\psi=\widetilde{\psi}$ if and only if $\psi$ is a solution to Problem $(\mathbf{L})$.

The most difficult component of $\psi$ to analyze is $\psi_{3}$. We treat this component first, and then briefly discuss the components $\psi_{1}$ and $\psi_{2}$

We may write $\psi_{3}=\eta_{1}(y) \Psi_{1}+\eta_{2}(y) \Psi_{2}+\Psi_{3}+\Psi_{4}+\Psi_{5}$ where $\eta_{i} \in C^{\infty}$,

$$
\begin{aligned}
& \eta_{1}(y)=\left\{\begin{array}{r}
1 \text { if } \frac{1}{4}<y \leq 1 \\
0 \text { if }-1 \leq y<-\frac{1}{4}
\end{array}\right. \\
& \eta_{2}(y)=1-\eta_{1}(y)
\end{aligned}
$$


and

$$
\begin{aligned}
& \Delta^{2} \Psi_{1}=\epsilon f_{3}(x, y) \text { for } x \in \mathbb{R}, y<1, \\
& \Psi_{1}=0 \text { at } y=1, \\
& \Psi_{1, y}=0 \text { at } y=1, x<0, \\
& \Psi_{1, y y}=0 \text { at } y=1, x>0 ; \\
& \Delta^{2} \Psi_{2}=\epsilon f_{3}(x, y) \text { for } x \in \mathbb{R}, y>-1, \\
& \Psi_{2}=0 \text { at } y=-1, \\
& \Psi_{2, y}=0 \text { at } y=-1, x<0, \\
& \Psi_{2, y y}=0 \text { at } y=-1, x>0 ; \\
& \Delta^{2} \Psi_{3}=-\Delta^{2}\left(\eta_{1}\right.\left.(y) \Psi_{1}+\eta_{2}(y) \Psi_{2}\right)+\eta_{1}(y) \Delta^{2} \Psi_{1}+\eta_{2}(y) \Delta^{2} \Psi_{2} \\
& \equiv \Psi_{3}=\quad \text { in }\{-1<y<1\}, \\
& \Psi_{3, y}=0 \text { at } y= \pm 1,= \pm 1,-\infty<x<0, \\
& \Psi_{3, y y}=0 \text { at } y= \pm 1,0<x<\infty ; \\
& \Psi_{2, y y}=0 \text { at } x>0, y= \pm 1, \\
& \Delta^{2} \Psi_{4}=0 \text { in }\{-1<y<1\} \\
& \Psi_{4}=0 \text { at } y= \pm 1, \\
& \Psi_{4, y}=-\Psi_{1, y} \text { at } x<0, y=1, \\
& \Psi_{4, y}=-\Psi_{2, y} \text { at } x<0, y=-1,
\end{aligned}
$$

and

$$
\begin{aligned}
\Delta^{2} \Psi_{5} & =0 \text { for }|x|<N,|y|<1, \\
\Psi_{5} & =-\sum_{i=1}^{4} \Psi_{i} \text { at } x= \pm N, \\
\Psi_{5, x} & =-\sum_{i=1}^{4} \Psi_{i, x} \text { at } x= \pm N, \\
\Psi_{5} & =0 \text { at } y= \pm 1, \\
\Psi_{5, y} & =0 \text { at }-N<x<0, y= \pm 1, \\
\Psi_{5, y y} & =0 \text { at } 0<x<N, y= \pm 1 .
\end{aligned}
$$

Observe that $f_{3}(x, y)$ (in $(11.15),(11.16)$ ) was defined only in $\Sigma$; we need to extend it to $\{|x|>N,|y| \leq 1\}$ in $C^{1+\alpha}$ way. We do it by defining it as constant along the horizontal lines $\{y=$ const; $x<-N\},\{y=$ const; $x>N\}$.

The existence of solutions $\Psi_{1}, \Psi_{2}$ such that

$$
\left\|\Psi_{i}\right\|_{\mathrm{C}_{2} 2+\alpha} \leq C\|T\|_{\mathrm{C}}^{\circ \alpha}
$$


was proved in the last two sections.

In order to solve problems (11.17), (11.18), (11.20) we use the theory developed in [6], [7], [9], [10] for problems with mixed boundary conditions. In particular, for problem (11.17) the Green function $K(x, y ; \xi, \eta)$ exists and its detailed asymptotic behavior near the points $(0, \pm 1)$ is known. In particular,

$$
K(x, y ; \xi, \eta)=\widetilde{K}(x-\xi, y-\eta)+R(x, y ; \xi, \eta)
$$

where

$$
\widetilde{K}(\vec{x}-\vec{\xi})=C|\vec{x}-\vec{\xi}|^{2} \log |\vec{x}-\vec{\xi}| \quad(C \text { is constant })
$$

and $R$ has a lower order singularity; if $|\eta| \leq \frac{3}{4}$ then the singularity of $R(x, y ; \xi, \eta)$ occurs only as $(x, y) \rightarrow(0, \pm 1)$. We can write

$$
\Psi_{3}(x, y)=\int_{\mathbb{R} \times[-1,1]} K(x, y ; \xi, \eta) W(\xi, \eta) d \xi d \eta
$$

It is important to recall that the support of $W(\xi, \eta)$ lies in $|\eta|<\frac{1}{2}$; furthermore

$$
W(\xi, \eta)=\eta_{1}(\eta) \frac{\partial^{3} \Psi_{1}}{\partial \eta^{3}}+\eta_{2}(\eta) \frac{\partial^{3} \Psi_{2}}{\partial \eta^{3}}+\widetilde{W}(\xi, \eta)
$$

where $\widetilde{W}(\xi, \eta)$ involves up to second order derivatives of $\Psi_{1}$ and $\Psi_{2}$. We can perform integration by parts in (11.20) with zero boundary contributions (as every derivative of $\Psi_{1}, \Psi_{2}$ is multiplied by $\eta_{j}$ or one of its derivatives) and obtain

$$
\begin{gathered}
\Psi_{3}(x, y) \\
=\int_{\mathbb{R} \times[-1,1]}\left(-\sum_{i=1}^{2} \frac{\partial\left[\eta_{i}(\eta) K(x, y ; \xi, \eta)\right]}{\partial \eta} \frac{\partial^{2} \Psi_{i}}{\partial \eta^{2}}+K(x, y ; \xi, \eta) \widetilde{W}(\xi, \eta)\right) d \xi d \eta .
\end{gathered}
$$

We may now use standard techniques to estimate the integrals and conclude that

$$
\left\|\Psi_{3}\right\|_{C^{2+\alpha}(\mathbb{R} \times[-1,1])} \leq C \sum_{i=1}^{2}\left\|\Psi_{i}\right\|_{C^{2+\alpha}\left(\mathbb{R} \times\left[-\frac{3}{4}, \frac{3}{4}\right]\right)} \leq \sum_{i=1}^{2}\left\|\Psi_{i}\right\|_{C_{2} 2+\alpha} .
$$

Moreover, the theory of $[6],[7],[9],[10]$ shows that near the point $(0,1)$ (resp. near $(0,-1))$,

$$
\Psi_{3}=\Gamma_{1} r^{\frac{3}{2}} f(\theta)+\widetilde{\Psi_{3}}
$$

where

$$
\left|\Gamma_{1}\right| \leq C \sum_{i=1}^{2}\left\|\Psi_{i}\right\|_{C_{2} 2+\alpha}
$$

and

$$
\left\|\widetilde{\Psi_{3}}\right\|_{C_{2} 2+\alpha} \leq C \sum_{i=1}^{2}\left\|\Psi_{i}\right\|_{C_{2}^{\circ 2+\alpha}}
$$


Note that, by symmetry with respect to the $x$-axis, the coefficient $\Gamma_{1}$ is the same at $(0,1)$ as at $(0,-1)$; the same observation applies to $\Psi_{i}, \psi_{j}$ below.

The above theory applies also to Problem (11.18), so that

$$
\Psi_{4}=\Gamma_{2} r^{\frac{3}{2}} f(\theta)+\widetilde{\Psi_{4}}
$$

where

$$
\begin{aligned}
& \left|\Gamma_{2}\right| \leq C \sum_{i=1}^{2}\left\|\Psi_{i, y}\right\|_{C_{1} 1+\alpha} \leq C \sum_{i=1}^{2}\left\|\Psi_{i}\right\|_{C_{2}{ }^{2+\alpha}}, \\
& \left\|\widetilde{\Psi_{4}}\right\|_{C_{2}{ }^{2+\alpha}} \leq C \sum_{i=1}^{2}\left\|\Psi_{i, y}\right\|_{C_{1}^{\circ 1+\alpha}} \leq C \sum_{i=1}^{2}\left\|\Psi_{i}\right\|_{C_{2}^{\circ 2+\alpha}} \text {. }
\end{aligned}
$$

We also have analogous results for the solution of (11.20), i.e.,

$$
\Psi_{5}=\Gamma_{3} r^{\frac{3}{2}} f(\theta)+\widetilde{\Psi_{5}}
$$

where

$$
\begin{aligned}
&\left|\Gamma_{3}\right| \leq C \sum_{i=1}^{4}\left\|\Psi_{i}\right\|_{C^{2+\alpha}} \leq C\left(\left|\Gamma_{1}\right|+\left|\Gamma_{2}\right|+\sum_{i=1}^{2}\left\|\Psi_{i}\right\|_{C_{2}^{2+\alpha}}+\sum_{i=3}^{4}\left\|\widetilde{\Psi}_{i}\right\|_{C_{C_{2}}^{2+\alpha}}\right), \\
&\left\|\widetilde{\Psi_{5}}\right\|_{\mathrm{O}_{2}^{2+\alpha}} \leq C \sum_{i=1}^{4}\left\|\Psi_{i}\right\|_{C^{2+\alpha}} \leq C\left(\left|\Gamma_{1}\right|+\left|\Gamma_{2}\right|+\sum_{i=1}^{2}\left\|\Psi_{i}\right\|_{\mathrm{O}_{2}^{2+\alpha}}+\sum_{i=3}^{4}\left\|\widetilde{\Psi}_{i}\right\|_{\mathrm{O}_{2}^{2+\alpha}}\right) .
\end{aligned}
$$

Summarizing, we have obtained a unique solution $\psi_{3}$ of (11.8) such that

$$
\psi_{3}=\eta\left(r_{1}\right) \Gamma_{4} r_{1}^{\frac{3}{2}} f\left(\theta_{1}\right)+\eta\left(r_{2}\right) \Gamma_{4} r_{2}^{\frac{3}{2}} f\left(\theta_{2}\right)+\overline{\psi_{3}}
$$

where $\left(r_{1}, \theta_{1}\right),\left(r_{2}, \theta_{2}\right)$ are polar coordinates near $(0,1)$ and $(0,-1)$ respectively and $\eta(r)$ is cutoff function which is equal to 1 for $r<\frac{1}{4}$ and to zero for $r>\frac{1}{2}$; furthermore, $\Gamma_{4}$ and $\overline{\psi_{3}}$ satisfy the estimates

$$
\begin{aligned}
\left|\Gamma_{4}\right| & \leq C\|T\|_{\stackrel{\circ}{\circ} \alpha}, \\
\left\|\overline{\psi_{3}}\right\|_{C_{2} 2+\alpha} & \leq C\|T\|_{C}^{\circ \alpha} .
\end{aligned}
$$

The same method can be applied to problem (11.7), and we obtain

$$
\psi_{2}=\eta\left(r_{1}\right) \Gamma_{5} r_{1}^{\frac{3}{2}} f\left(\theta_{1}\right)+\eta\left(r_{2}\right) \Gamma_{5} r_{2}^{\frac{3}{2}} f\left(\theta_{2}\right)+\overline{\psi_{2}}
$$

where

$$
\begin{aligned}
\left|\Gamma_{5}\right| & \leq C|\epsilon|\left\|T_{0}\right\|_{C} \alpha \\
\left\|\overline{\psi_{2}}\right\|_{C_{2}^{\circ 2+\alpha}} & \leq C|\epsilon|\left\|T_{0}\right\|_{C}^{\circ \alpha} .
\end{aligned}
$$

We next consider Problem (11.6). Since the right hand side of the differential equation consists of the term $\left(-\Delta^{2} \psi_{0}\right)$ and of terms involving only second 
derivatives of $\widetilde{\psi}$, the theory of $[6],[7],[9],[10]$ can again be applied to conclude that

$$
\psi_{1}=\eta\left(r_{1}\right) \Gamma_{6} r_{1}^{\frac{3}{2}} f\left(\theta_{1}\right)+\eta\left(r_{2}\right) \Gamma_{6} r_{2}^{\frac{3}{2}} f\left(\theta_{2}\right)+\overline{\psi_{1}}
$$

where

$$
\begin{aligned}
\left|\Gamma_{6}\right| & \leq C\left(|\epsilon|+\left|\frac{\alpha_{1}}{\alpha_{2}}\right| \operatorname{Re}\right)\|\widetilde{\psi}\|_{\mathrm{o}_{2}^{2+\alpha}}, \\
\left\|\overline{\psi_{1}}\right\|_{C_{C_{2}} 2+\alpha} & \leq C\left(|\epsilon|+\left|\frac{\alpha_{1}}{\alpha_{2}}\right| \operatorname{Re}\right)\|\widetilde{\psi}\|_{\mathrm{o}_{2}^{2+\alpha}} .
\end{aligned}
$$

Finally, the solution $\psi_{4}$, of (11.9) with boundary conditions (11.10), (11.11), (11.13), (11.14), can be written (using the theory of [6], [7], [9], [10], as well as the estimates of Sections 4-8) in the form

$$
\psi_{4}=\eta\left(r_{1}\right) \Gamma_{7} r_{1}^{\frac{3}{2}} f\left(\theta_{1}\right)+\eta\left(r_{2}\right) \Gamma_{7} r_{2}^{\frac{3}{2}} f\left(\theta_{2}\right)+\overline{\psi_{4}}
$$

where

$$
\begin{aligned}
&\left|\Gamma_{7}\right| \leq C\|G[\widetilde{\psi}]\|_{C^{\alpha}} \leq C|\epsilon|\left(1+\|\widetilde{\psi}\|_{C_{2}^{2+\alpha}}\right), \\
&\left\|\overline{\psi_{4}}\right\|_{C_{2}^{2} 2+\alpha} \leq C\|G[\widetilde{\psi}]\|_{C^{\alpha}} \leq C|\epsilon|\left(1+\|\widetilde{\psi}\|_{O_{C_{2}}^{2+\alpha}}\right) .
\end{aligned}
$$

\section{Part IV}

\section{Solution of Problem (L); More general Oldroyd models}

\section{The fixed point}

In this section we shall establish the existence of a solution to Problem $(L)$ in a neighborhood of the flow with stream function $\psi_{0}$. The solution will belong to a class of functions of the form

$$
\psi=\eta\left(r_{1}\right) \Gamma r_{1}^{\frac{3}{2}} f\left(\theta_{1}\right)+\eta\left(r_{2}\right) \Gamma r_{2}^{\frac{3}{2}} f\left(\theta_{2}\right)+\psi_{0}+\widetilde{\psi}
$$

where $\Gamma$ is a real number, $\widetilde{\psi} \in \stackrel{\circ}{C}_{2}^{2+\alpha}(\Sigma)$ and

$$
|\Gamma|+\|\widetilde{\psi}\|_{C_{2}^{2+\alpha}(\Sigma)} \leq M \quad(M>1)
$$

We denote by $X$ the Banach space of pairs $(\Gamma, \widetilde{\psi})$ with norm

$$
\|(\Gamma, \widetilde{\psi})\|_{X}=|\Gamma|+\|\widetilde{\psi}\|_{\mathrm{O}_{2}^{2}(\Sigma)}^{2+\alpha},
$$

and by $X_{M}$ the ball of radius $M$ in $X$. By the results of Section 11, for any

$$
\bar{\psi}=\eta\left(r_{1}\right) \bar{\Gamma} r_{1}^{\frac{3}{2}} f\left(\theta_{1}\right)+\eta\left(r_{2}\right) \bar{\Gamma} r_{2}^{\frac{3}{2}} f\left(\theta_{2}\right)+\psi_{0}+\widetilde{\bar{\psi}}
$$


with $(\bar{\Gamma}, \widetilde{\bar{\psi}}) \in X_{M}$, there exists a unique solution $\psi$ of Problem (11.1)-(11.5) in the form (12.1) and

$$
|\Gamma|+\|\widetilde{\psi}\|_{C_{2}^{2+\alpha}(\Sigma)} \leq C\left(\left\|\psi_{0}\right\|_{C_{2}^{2+\alpha}(\Sigma)}+\left(|\epsilon|+\left|\frac{\alpha_{1}}{\alpha_{2}}\right| \operatorname{Re}\right)\left(|\bar{\Gamma}|+\|\widetilde{\widetilde{\psi}}\|_{C_{2}^{\circ 2+\alpha}(\Sigma)}\right)\right)
$$

Introducing the mapping $W$ by

$$
W(\bar{\Gamma}, \widetilde{\bar{\psi}})=(\Gamma, \widetilde{\psi})
$$

we see that $W$ maps $X_{M}$ into itself provided

$$
M=C\left\|\psi_{0}\right\|_{C_{2}^{2+\alpha}(\Sigma)}+1
$$

and $|\epsilon|+$ Re is sufficiently small (note that $\left|\frac{\alpha_{1}}{\alpha_{2}}\right| \sim 1$ if $|\epsilon|$ is small).

By linearity of the problem, given two pairs $\left(\bar{\Gamma}_{1}, \widetilde{\bar{\psi}}_{1}\right)$ and $\left(\bar{\Gamma}_{2}, \widetilde{\bar{\psi}}_{2}\right)$ in $X_{M}$ their images $\left(\Gamma_{1}, \widetilde{\psi}_{1}\right)$ and $\left(\Gamma_{2}, \widetilde{\psi}_{2}\right)$ under the mapping $W$ satisfy

$$
\left|\Gamma_{1}-\Gamma_{2}\right|+\left\|\widetilde{\psi}_{1}-\widetilde{\psi}_{2}\right\|_{C_{2} 2+\alpha} \leq C(|\epsilon|+\operatorname{Re})\left(\left|\bar{\Gamma}_{1}-\bar{\Gamma}_{2}\right|+\left\|\widetilde{\bar{\psi}}_{1}-\widetilde{\bar{\psi}}_{2}\right\|_{C_{2} 2+\alpha}\right) .
$$

Hence if $|\epsilon|+\operatorname{Re}$ is sufficently small then $W$ is a contraction. Therefore, by Banach's fixed point theorem we conclude:

Theorem 12.1 If $|\epsilon|$ and Re are small enough, then there exists a unique solution of Problem (L) in the form (12.1) with

$$
|\Gamma|+\|\widetilde{\psi}\|_{C_{2}^{2+\alpha}} \leq C(|\epsilon|+\operatorname{Re}) .
$$

Remark 12.1. From the form of $\psi$ in we see that although the first order term in the expansion corresponds to a Newtonian singularity, some of the second order terms (although belonging to $\stackrel{\circ}{C}_{2}^{2+\alpha}$ ) are genuinely non-Newtonian. Indeed, as we have seen in the analysis of Sections 3 and 4, there appear second order terms with coefficients of the form $\operatorname{Re}\left\{(\pi-\theta)^{ \pm \frac{3}{8} \sqrt{b^{\prime}} i}\right\}$ as $\theta \rightarrow \pi$, and these are highly oscillatory.

Remark 12.2. Theorem 12.1 can be extended to somewhat more general $\overrightarrow{v_{0}}$. In particular, the requirement $(2.22)$ may be replaced by

$$
\psi=C r^{\frac{3}{2}}\left(\cos \frac{3 \theta}{2}-\cos \frac{\theta}{2}\right)(1+e(r, \theta))
$$

where $e(r, \theta)$ is an error terms such that $|e(r, \theta)| \leq C r$. This is the same error term as for the stick-slip problem for Newtonian flow (cf. [12]). 


\section{Discussion of Jeffrey's model}

In this section we discuss the model corresponding to $a=c=d=0$; then also $b^{\prime}=b=0$. This case corresponds to the so called Jeffrey model (see [2], for instance). All the estimates deduced in Sections 4-7 hold in this case except for those in the region $\theta_{f} \in\left(\theta_{2}, \pi\right)$ which involve the integral $I_{6}$ of Lemma 4.3 (and the corresponding integral with $D_{0}$ substituted by $\widetilde{D}$ ). In order to analize $I_{6}$ in this region, let us define

$$
\begin{aligned}
T^{\prime}= & \left(\begin{array}{c}
T_{x x}+T_{y y} \\
T_{x x}-T_{y y} \\
T_{x y}
\end{array}\right), \\
D^{\prime}= & \left(\begin{array}{c}
D_{x x}+D_{y y} \\
D_{x x}-D_{y y} \\
D_{x y}
\end{array}\right)=\left(\begin{array}{c}
0 \\
2 D_{x x} \\
D_{x y}
\end{array}\right) .
\end{aligned}
$$

Then the hyperbolic system can be written as

$$
K^{\prime} T^{\prime}=T^{\prime}+\alpha_{1}\left(\frac{d}{d s} T^{\prime}+A^{\prime}\left(\overrightarrow{v_{0}}\right) T^{\prime}\right)=\epsilon D^{\prime},
$$

where

$$
A^{\prime}\left(\overrightarrow{v_{0}}\right)=\left(\begin{array}{ccc}
0 & 0 & 0 \\
0 & 0 & 2\left(\frac{\partial v_{0, y}}{\partial x}-\frac{\partial v_{0, x}}{\partial y}\right) \\
0 & -\left(\frac{\partial v_{0, y}}{\partial x}-\frac{\partial v_{0, x}}{\partial y}\right) & 0
\end{array}\right)
$$

and therefore

$$
\left(T_{x x}+T_{y y}\right)(s)=\left(T_{x x}+T_{y y}\right)\left(s_{0}\right) e^{-\frac{\left(s-s_{0}\right)}{\alpha_{1}}},
$$

which yields uniform boundedness of $T_{x x}+T_{y y}$.

As in Section 3, we can write

$$
T^{\prime}=\left(\begin{array}{c}
T_{x x}-T_{y y} \\
T_{x y}
\end{array}\right)=U V
$$

with $U$ a $2 \times 2$ matrix and $V$ a vector of two components. $U$ satisfies

$$
\frac{d U}{d s}+\left(\frac{\partial v_{0, y}}{\partial x}-\frac{\partial v_{0, x}}{\partial y}\right)\left(\begin{array}{cc}
0 & -2 \\
1 & 0
\end{array}\right) U=0
$$

and, in the region we are interested in (i.e., $\theta_{f} \in\left(\theta_{2}, \pi\right)$ ), we can write

$$
\frac{d U}{d s}-2 D_{0, x y}\left(\begin{array}{cc}
0 & -2 \\
1 & 0
\end{array}\right) U=0 .
$$

Defining $d s^{\prime}=\frac{1}{2 D_{0, x y}} d s$, and diagonalyzing, we obtain by the techniques of Section 3,

$$
U=M^{-1}\left(\begin{array}{cc}
e^{\sqrt{2} i s^{\prime}} & 0 \\
0 & e^{-\sqrt{2} i s^{\prime}}
\end{array}\right) M C
$$


for some constant matrices $M$ and $C$. We then deduce that the integral $I_{6}$ is a linear combination with bounded coefficients of the integrals

$$
K_{ \pm}(s)=\int_{s_{0}}^{s} e^{ \pm \sqrt{2} i s^{\prime}(s)} e^{-\frac{s}{\alpha_{1}}} D d s
$$

If we change variables first from $s$ to $\theta$ and then from $\theta$ to $u=-\log (\pi-\theta)$ we find (similarly to (4.32), (4.33)) that $K_{ \pm}(s)$ take the form

$$
K_{ \pm}=\int_{-\log \left(\pi-\theta_{2}\right)}^{-\log \left(\pi-\theta_{f}\right)} e^{\sqrt{2} i s^{\prime}(u)} e^{-\rho e^{-\frac{1}{3} u}} d u \equiv K_{ \pm}\left(\rho, \theta_{f}\right),
$$

or

$$
K_{ \pm}\left(\rho, \theta_{f}\right)=\int_{-\log \left(\pi-\theta_{2}\right)}^{-\log \left(\pi-\theta_{f}\right)} e^{\sqrt{2} i s^{\prime}(u)} e^{-\rho e^{-\frac{1}{3} u}} e^{-u} d u
$$

here we consider for simplicity just the highest order terms of $D$. It can be easily verified that $s^{\prime}(u) \rightarrow 0$ as $u \rightarrow \infty$, and, therefore,

$$
K_{ \pm}\left(\rho, \theta_{f}\right) \sim-\log \left(\pi-\theta_{f}\right) \quad \text { as } \theta_{f} \rightarrow \pi, \text { in case (13.4) }
$$

and

$$
K_{ \pm}\left(\rho, \theta_{f}\right) \sim C \quad \text { as } \theta_{f} \rightarrow \pi, \text { in case (13.5). }
$$

We conclude that

$$
\begin{aligned}
T_{x x}-T_{y y} & \sim C_{1} \log \left(\pi-\theta_{f}\right) \quad \text { as } \theta_{f} \rightarrow \pi, \\
T_{x y} & \sim C_{2} \log \left(\pi-\theta_{f}\right) \quad \text { as } \theta_{f} \rightarrow \pi .
\end{aligned}
$$

Recalling that $T_{x x}+T_{y y}$ is uniformly bounded, it follows that

$$
T \sim C \log \left(\pi-\theta_{f}\right) \quad \text { if } \theta_{f} \rightarrow \pi
$$

where $C$ is a constant vector. Similarly one can estimate the Hölder coefficients of $T$ and thus establish an equivalent to Theorem 8.4 for $T-C \log \left(\pi-\theta_{f}\right)$. If we define

$$
T=T^{(1)}+\widehat{T}
$$

where

$$
T^{(1)}=C \eta(\theta) \log (\pi-\theta)
$$

and

$$
\eta(\theta) \in C^{\infty}, \eta^{\prime}(\theta) \geq 0 \text { and } \eta(\theta)=\left\{\begin{array}{cc}
1 & \text { if } \theta \in\left[\frac{2 \theta_{2}}{3}, \pi\right] \\
0 & \text { if } \theta<\frac{\theta_{2}}{3}
\end{array},\right.
$$

then the conclusion of Theorem 8.4 holds also for $\widehat{T}$.

For the Jeffrey's model the boundary condition (2.21) becomes

$$
\frac{1}{r^{2}} \psi_{\theta \theta}-\frac{1}{\epsilon-1} T_{x y} \rightarrow 0 \text { as } \theta \rightarrow \pi \text { for any } r>0 .
$$

Setting

$$
\psi^{(1)}=\frac{r^{2}}{\epsilon-1} \eta(\theta) \frac{(\pi-\theta)^{2}}{2} T_{x y}^{(1)}
$$


and introducing a function $\widehat{\psi}$ by

$$
\psi=\psi^{(1)}+\widehat{\psi},
$$

one can extend the analysis of Sections 9,10 to the system (11.1)-(11.5) for $\widehat{\psi}$ (instead of $\psi$ ) and establish the existence of a unique solution $\widehat{\psi}$ which yields a unique solution to Problem (L) with $T$ and $\psi$ of the form (13.6), (13.8).

Although the velocity field $\left(\psi_{y},-\psi_{x}\right)$ about $(0, \pm 1)$ is Newtonian, the second derivative of $\psi$ is non Newtonian; in fact

$$
\frac{1}{r^{2}} \psi_{\theta \theta} \sim C \log (\pi-\theta)(C \neq 0) \text { as } \theta \rightarrow \pi, r<<1,
$$

whereas for Newtonian flow

$$
\frac{1}{r^{2}} \psi_{\theta \theta} \sim o(1) \text { as } \theta \rightarrow \pi, r<<1 .
$$

Notice that (13.9) coincides with (1.20).

The relation (13.9) implies a singular strain $D_{x y}$ along the slip boundary.

\section{The solution for general Oldroyd models}

In this section we consider the case $d \neq a$. In this case the solution of the hyperbolic Problem $\left(\mathbf{H}_{0}\right)$ develops a new type of singularity and, as will be formally shown, a non-Newtonian singularity will form near the linearized free boundary. Going over the calculation of $I\left(\theta_{0}, \widetilde{\theta_{f}}\right)$ in Section 4 (corresponding to some pair $\left(\widetilde{r_{f}}, \widetilde{\theta_{f}}\right)$ ) we see that, when $d \neq a$, in addition to linear combinations of

$$
u^{-1-\frac{2}{3} b} e^{ \pm \frac{4}{3 \widetilde{\theta_{f}}}(u-1) i},{\widetilde{\theta_{f}}}^{-1} u^{-\frac{2}{3} b} e^{ \pm \frac{4}{3 \widetilde{\theta_{f}}}(u-1) i}
$$

there also appears, linearly, the term $u^{\frac{4}{3} b-1}$. Thus, in addition to integrals $J, K$ as in (4.13), (4.14), there appear integrals

$$
L=\int_{1}^{\frac{\widetilde{\theta_{f}}}{\theta_{0}}} e^{-\rho\left(u^{\frac{5}{3}}-1\right)\left(1+O\left(\theta^{2}\right)\right)} u^{\frac{4}{3} b-1} d u
$$

where $\rho=\frac{2}{5 \alpha_{1}} \frac{\widetilde{r}_{f}^{\frac{1}{2}}}{\widetilde{\theta}_{f}}$. Here $b$ is defined as in (1.18), and we consider the case $0<b<\frac{3}{2}$ so that $J$ and $K$ ccan be analyzed exactly as before.

If we fix $\widetilde{\theta}_{f}=\theta_{1}$ then, for fixed $\theta_{0}<\theta_{1}$ if $r_{0}$ approaches zero, $\widetilde{r}_{f}$ also approaches 0 (see Figure 2) so that

$$
\begin{aligned}
& L \sim \int_{1}^{\infty} e^{-\rho\left(u^{\frac{5}{3}}-1\right)} u^{\frac{4}{3} b-1} d u \\
& =\frac{3}{5} \int_{1}^{\infty} e^{-\rho(v-1)} v^{\frac{4}{5} b-1} d v=\frac{3}{5} e^{\rho} \rho^{-\frac{4}{5} b} \int_{\rho}^{\infty} e^{-v} v^{\frac{4}{5} b-1} d v \sim C \rho^{-\frac{4}{5} b} \text { as } \rho \rightarrow 0
\end{aligned}
$$




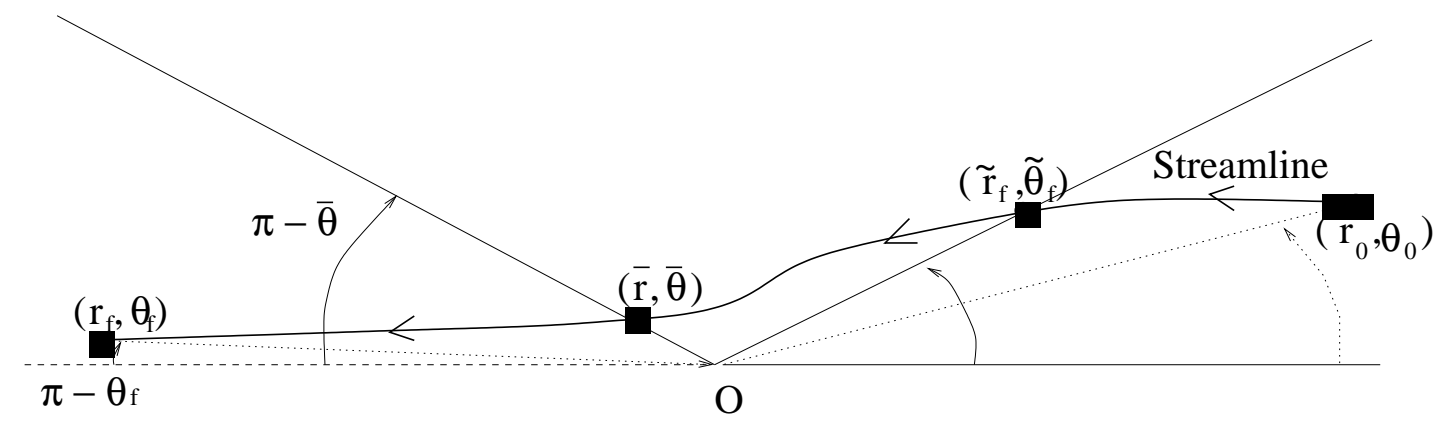

Figure 2: The geometry of the streamlines near the point $(0,1)$

in contrast with the fact that both $J$ and $K$ remain bounded (as proved in Section 4).

Tracing this singularity to $T$, we find that

$$
\begin{aligned}
& T_{x x} \sim \epsilon C_{1}(\theta) \widetilde{r}_{f}^{-\frac{2}{5} b}, \\
& T_{y y} \sim \epsilon C_{2}(\theta) \widetilde{r}_{f}^{-\frac{2}{5} b} \text { for } \theta \text { small but fixed, and } \widetilde{r_{f}} \rightarrow 0 .
\end{aligned}
$$

This singularity is convected along the Newtonian streamlines of $\overrightarrow{v_{0}}$ (which "mixes" the components of $T$ ) so that

$$
\begin{aligned}
T_{x x} & \sim \epsilon C_{1}(\theta) r^{-\frac{2}{5} b}, T_{y y} \sim \epsilon C_{2}(\theta) r^{-\frac{2}{5} b}, T_{x y} \sim \epsilon C_{3}(\theta) r^{-\frac{2}{5} b} \\
\text { for every } \theta & <\pi, \text { as } r \rightarrow 0
\end{aligned}
$$

where $C_{1}(\theta), C_{2}(\theta), C_{3}(\theta)$ are bounded for each $\theta$. As we approach the angle $\theta=\pi$, the Newtonian streamlines, to first approximation, satisfy

$$
r^{\frac{3}{2}} \vartheta=\text { Const. where } \vartheta=\pi-\theta .
$$

If we take as reference the line $\theta=\bar{\theta}$ with $\bar{\theta}$ close to $\pi$ (but fixed, see Figure 2 ), and define the distance of a point on it to the origin by $\bar{r}$, then we have

$$
r_{f}^{\frac{3}{2}} \vartheta_{f}=\bar{r}^{\frac{3}{2}}(\pi-\bar{\theta}) \quad\left(\vartheta_{f}=\pi-\theta_{f}\right)
$$

so that

$$
\bar{r}=C r_{f} \vartheta_{f}^{\frac{2}{3}}
$$


and, therefore, from the estimates (14.2) with $\theta=\bar{\theta}$ and $r=\bar{r}$ we see that the vector $T$ will have a singularity of the form

$$
T\left(r_{f}, \vartheta_{f}\right) \sim \epsilon C(\bar{\theta})\left(r_{f} \vartheta_{f}^{\frac{2}{3}}\right)^{-\frac{2}{5} b} .
$$

This singularity, distributed (near the origin) along the line $\vartheta_{f}=0$, indicates the failure of the Newtonian approximation in the region $\vartheta_{f}<<1$ (since the Newtonian singularity occurs only at the origin $O$ ). Nevertheless we shall assume that the velocity field $\left(\psi_{y},-\psi_{x}\right)$ is Newtonian (but not the second derivatives of $\psi$ !) and verify this hypothesis a posteriori.

If we keep the dominant terms in equation (2.18), which correspond ot the higher order derivatives in $\vartheta$ for $\psi$ and $T_{x y}$, we obtain the following equation (written in polar coordinates):

$$
\frac{1}{r^{4}} \frac{\partial^{4} \psi}{\partial \vartheta^{4}}=\frac{1}{\epsilon-1} \frac{1}{r^{2}} \frac{\partial^{2} T_{x y}}{\partial \vartheta^{2}}
$$

where $T_{x y}$ is given by (14.3). By the boundary condition, as $\vartheta \rightarrow 0$ the matrix $A\left(\overrightarrow{v_{0}}\right)$ in $(2.2)$ is approximately

$$
A\left(\vec{v}_{0}\right)=\left(\begin{array}{ccc}
-2 a+c+2 d & c-2 d & 0 \\
-c+2 d & 2 a-c-2 d & 0 \\
0 & 0 & 0
\end{array}\right) \frac{\partial v_{0, x}}{\partial x}
$$

Therefore, in the system for $T$, the equation for $T_{x y}$ is uncoupled to the equations for $T_{x x}$ and $T_{y y}$. The equation for $T_{x y}$, to a leading order, is

$$
T_{x y}+\alpha_{1} \frac{d T_{x y}}{d s}=\epsilon D_{x y} .
$$

If we use polar coordinates and the definition of $\tau_{(1)}$ as given in (1.4), then we may write (to a leading order) equation (14.5) in the form

$$
T_{x y}-\alpha_{1}\left[\frac{1}{r} \psi_{\vartheta} \frac{\partial T_{x y}}{\partial r}-\frac{1}{r} \psi_{r} \frac{\partial T_{x y}}{\partial \vartheta}\right]=\epsilon\left(\frac{1}{r^{2}} \psi_{\vartheta \vartheta}-\psi_{r r}\right)
$$

(note that $\partial_{\theta}=-\partial_{\vartheta}$ ).

From (14.4) we obtain, after integration,

$$
\frac{1}{r^{2}} \psi_{\vartheta \vartheta}=\frac{1}{\epsilon-1}\left(T_{x y}+B(r)\right)+A(r) \vartheta
$$

The boundary condition

$$
\frac{1}{r^{2}} \psi_{\vartheta \vartheta}-\frac{1}{\epsilon-1} T_{x y} \rightarrow 0 \text { as } \vartheta \rightarrow 0,
$$

implies that $B(r)=0$. When $\epsilon \rightarrow 0, \psi$ becomes a Newtonian stream function, and this implies that

$$
A(r)=C r^{-\frac{1}{2}}(C \text { constant } \neq 0)
$$


Hence (14.7) reduces to

$$
\frac{1}{r^{2}} \psi_{\vartheta \vartheta}=\frac{1}{\epsilon-1} T_{x y}+C r^{-\frac{1}{2}} \vartheta
$$

If we combine now (14.8) with (14.6), we obtain, to a leading order, the following equation:

$$
\frac{1}{1-\epsilon} T_{x y}-\alpha_{1}\left[\frac{1}{r} \psi_{\vartheta} \frac{\partial T_{x y}}{\partial r}-\frac{1}{r} \psi_{r} \frac{\partial T_{x y}}{\partial \vartheta}\right]=\epsilon C r^{-\frac{1}{2}} \vartheta .
$$

This hyperbolic equation can be solved for $\theta_{1}<\theta<\pi$ provided $\psi_{\vartheta}, \psi_{r}$ and $\left.T_{x y}\right|_{\theta=\theta_{1}}$ are given functions; here $\theta_{1} \in(0, \pi)$ and $\pi-\theta_{1}<<1$. Since we assume that the velocity field is Newtonian, we can, for $\theta_{1}<\theta<\pi$,

$$
\frac{1}{1-\epsilon} T_{x y}+\alpha_{1} \frac{d T_{x y}}{d s}=C \epsilon r^{-\frac{1}{2}}(\pi-\theta)
$$

where, to a leading order,

$$
d s=\frac{2}{3} \frac{r^{\frac{1}{2}}(\theta)}{\pi-\theta} d \theta
$$

so that

$$
\begin{gathered}
T_{x y}\left(r_{f}, \theta_{f}\right)=e^{-\frac{2}{3} \frac{1}{(1-\epsilon) \alpha_{2}} \int_{\theta_{1}}^{\theta_{f}} \frac{r^{\frac{1}{2}}(\theta)}{\pi-\theta} d \theta} T_{x y}\left(r, \theta=\theta_{1}\right) \\
+C \epsilon(1-\epsilon) \int_{\theta_{1}}^{\theta_{f}} e^{-\frac{2}{3} \frac{1}{(1-\epsilon) \alpha_{2}} \int_{\theta_{1}}^{\eta} \frac{r^{\frac{1}{2}}(\theta)}{\pi-\theta} d \theta} r^{-\frac{1}{2}}(\eta)(\pi-\eta) \frac{r^{\frac{1}{2}}(\eta)}{\pi-\eta} d \eta
\end{gathered}
$$

Since

$$
\begin{aligned}
r^{\frac{3}{2}}(\theta)(\pi-\theta) \sim r_{f}^{\frac{3}{2}}\left(\pi-\theta_{f}\right), \\
\int_{\theta_{1}}^{\theta_{f}} \frac{r^{\frac{1}{2}}(\theta)}{\pi-\theta} d \theta=r_{f}^{\frac{1}{2}}\left(\pi-\theta_{f}\right)^{\frac{1}{3}} \int_{\theta_{1}}^{\theta_{f}} \frac{1}{(\pi-\theta)^{\frac{4}{3}}} d \theta \\
=-3 r_{f}^{\frac{1}{2}}\left(\pi-\theta_{f}\right)^{\frac{1}{3}}\left(\frac{1}{\left(\pi-\theta_{f}\right)^{\frac{1}{3}}}-\frac{1}{\left(\pi-\theta_{1}\right)^{\frac{1}{3}}}\right) \\
=-3 r_{f}^{\frac{1}{2}}\left(\pi-\theta_{f}\right)^{\frac{1}{3}}(1+o(1))
\end{aligned}
$$

and

$$
\begin{gathered}
\int_{\theta_{1}}^{\theta_{f}} e^{-\frac{2}{3} \frac{1}{(1-\epsilon) \alpha_{2}} \int_{\theta_{1}}^{\vartheta} \frac{r^{\frac{1}{2}}(\theta)}{\pi-\theta} d \theta} r^{-\frac{1}{2}}(\vartheta)(\pi-\vartheta) \frac{r^{\frac{1}{2}}(\vartheta)}{\pi-\vartheta} d \vartheta \\
\sim \int_{\theta_{1}}^{\theta_{f}} e^{-\frac{2}{(1-\epsilon) \alpha_{2}} r_{f}^{\frac{1}{2}}(\pi-\vartheta)^{\frac{1}{3}}\left(\frac{1}{(\pi-\vartheta)^{\frac{1}{3}}}-\frac{1}{\left(\pi-\theta_{1}\right)^{\frac{1}{3}}}\right)} d \vartheta \leq C .
\end{gathered}
$$

Hence the second term at the right-hand side of (14.9) is bounded whereas, in the first term, we use the boundedness of the integral and the relations (14.3), (14.10) to get

$$
T_{x y}(r, \theta)=\widetilde{C} \epsilon r^{-\frac{2}{5} b}(\pi-\theta)^{-\frac{4}{15} b}
$$


where $\widetilde{C}$ is a bounded function, $\widetilde{C} \rightarrow C_{0} \neq 0$ if $\theta \rightarrow \pi$. By (14.8) we can deduce that

$$
\psi_{\theta \theta}=\frac{\widetilde{C} \epsilon}{\epsilon-1} r^{2-\frac{2}{5} b}(\pi-\theta)^{-\frac{4}{15} b}+C r^{\frac{3}{2}}(\pi-\theta)
$$

and by integration

$$
\begin{aligned}
\psi= & \frac{\widetilde{C} \epsilon}{\epsilon-1} r^{2-\frac{2}{5} b} \frac{(\pi-\theta)^{2-\frac{4}{15} b}}{\left(2-\frac{4}{15} b\right)\left(1-\frac{4}{15} b\right)}-D(r)(\pi-\theta) \\
& +\frac{C}{6} r^{\frac{3}{2}}(\pi-\theta)^{3}
\end{aligned}
$$

If $\epsilon \rightarrow 0$ we have to recover the Newtonian singularity, so that $D(r)=C_{2} r^{\frac{3}{2}}, C_{2}$ constant.

From (14.13) we see that

$$
\nabla \psi=\operatorname{Const} \nabla\left[r^{\frac{3}{2}}(\pi-\theta)(1+o(1))\right]
$$

where $o(1)$ comes form the first term on the right-hand side of (14.13). Hence the velocity field is Newtonian, as we have assumed above. The first term at the right-hand side of (14.12) is the principal singularity of $\psi_{\theta \theta}$ as $\theta \rightarrow \pi$, and is non-Newtonian; the second term on the right-hand side of (14.12) is the Newtonian contribution to $\psi_{\theta \theta}$. Note that (14.12) implies (1.21).

Remark 14.1. If $b<0,|b|$ small, then the integrals $J, K$ defined in (4.13) and $L$ defined in (14.1) are all bounded. Nevertheless, we cannot conclude that the integral $L_{1}\left[W ; r_{f}, \theta_{f}\right]$ of Lemma 6.1 in Section 6 is bounded and therefore, a boundary layer of the type described above could appear.

If $b^{\prime}<0$ but $\left|b^{\prime}\right|$ is small, then the matrix $U(\theta)$ defined in Theorem 3.3 contains a singularity of the form $|\pi-\theta|^{-\frac{8}{3} \sqrt{\left|b^{\prime}\right|}}$ as $\theta \rightarrow \pi$. Nevertheless, if $\sqrt{\left|b^{\prime}\right|}<\frac{3}{8}$, then the component $T_{x y}$ remains uncoupled (to higher order) to the components $T_{x x}$ and $T_{y y}$ of the stress tensor and the assumptions that led to equations (14.4), (14.5) remain valid. Hence the analysis of the boundary layer done in this section can be extended to this case.

\section{Conclusions}

In this paper we studied the stick-slip problem in a strip $|y|<1$ for nonNewtonian flows of Oldroyd type in the Johnson-Segalman form; the flow emerges for $x<0$ toward $x>0$, so that the stick-slip contact points are at $(0, \pm 1)$. The non-Newtonian model depends on three parameters: $a, c$ and $d$. Under the assumption of Newtonian kinematics, i.e., replacing the constitutive equations (1.3) (1.4) by (1.5)-(1.7), we analyzed the case $d=a, b=a^{2}-a c>0$ and proved that the singularity at $(0, \pm 1)$ is to a leading order of Newtonian type; however, the next order term shows a very oscillatory (non-Newtonian) character.

Next we have shown that as soon as $d \neq a$ (as well as in the case $d=a=c=$ 0 which corresponds to Jeffrey's model) the leading order singularity at $(0, \pm 1)$ 
is non-Newtonian and is such that the component $D_{x y}$ of the strain tensor becomes singular along the free boundary. We have also given a description of this singularity. This result was established under the assumption that $0<$ $b<\frac{3}{2}$. But, as indicated at the end of Section 14, this type of non-Newtonian singularity should occur also in cases where $b$ or $b^{\prime}$ (defined in (1.18) and (1.18)) are negative and small in absolute value.

\section{ACKNOWLEDGMENTS}

The first author is grateful for the support from the Institute of Mathematics and its Applications and has been partially supported by the Spanish Ministry of Education. The second author is partially supported by National Science Foundation Grant DMS \#9970522.

\section{References}

[1] M. R. Apelian, R. C. Armstrong and R. A. Brown. Impact of the constitutive equation and singularity on the calculation of stick-slip flow: the modified upper-convected Maxwell model (MUCM). J. non-Newtonian Fluid Mechanics, 27 (1988), 299-321.

[2] R. B. Bird, R. C. Armstrong, O. Hassager. Dynamics of polymeric liquids, Vol. 1. Wiley, New York, 1977.

[3] M. A. Fontelos, A. Friedman. Stationary non-Newtonian flows in channellike and pipe-like domains. To appear in Archive for Rational Mechanics and Analysis.

[4] D. Gilbarg, N. S. Trudinger. Elliptic partial differential equations of second order. Second edition. Gründlehren del Mathematischen Wissenschaften, 224. Springer-Verlag, Berlin-New York, 1983.

[5] D. D. Joseph. Fluid dynamics of viscoelastic liquids. Applied Mathematical Sciences, 84. Springer-Verlag, New York, 1990.

[6] V. A. Kondrat'ev, Boundary value problems for elliptic equations in domains with conical or angular points, Trudy Mosk. Mat. Obsch., 16 (1967), 209-292. English transl.: Trans. Moscow Math. Soc., 16 (1967), 227-313.

[7] V. A. Kozlov, V. G. Maz'ya and J. Rossmann, Elliptic boundary problems in domains with point singularities, Mathematical Surveys and Monographs, Vol. 52, American Mathematical Society, Providence R. I. (1997).

[8] R. G. Larson. Instabilities in viscoelastic flows. Rheologica Acta, 31 (1992), 213-263.

[9] V. G. Maz'ya, N. F. Mozorov, B. A. Plamenevskii and L. Stupyalis, Elliptic Boundary Value Problems, American mathematical society translations, series 2, Vol. 123, (1984). 
[10] B. A. Plamenevskij, Elliptic boundary value problems in domains with piecewise smooth boundary, In Encyclopaedia of Mathematical Sciences, Vol. 79, Springer-Verlag Berlin (1997).

[11] M. Renardy. The stresses of an upper convected Maxwell fluid in a Newtonian velocity field near a re-entrant corner. J. non-Newtonian Fluid Mechanics, 50 (1993), 127-134.

[12] S. Richardson. A "stick-slip" problem related to the motion of a free jet at low Reynolds numbers. Proc. Cambridge Philos. Soc., 67 (1970), 477-489.

[13] T. R. Salamon, D. E. Bornside, R. C. Armstrong and R. A. Brown. Local similarity solutions for the stress filed in the presence of a slip boundary condition. Phys. Fluids, 9 (1997), 1235-1247.

[14] T. R. Salamon, D. E. Bornside, R. C. Armstrong and R. A. Brown. Local similarity solutions for the stress filed of an Oldroyd-B fluid in the partial slip/slip flow. Phys. Fluids, 9 (1997), 2191-2209.

[15] V. A. Solonnikov. Problème de frontiére libre dans l'écoulement d'un fluide á la sortie d'un tube cylindrique. Asymptotic analysis 17 (1998), 135-163.

[16] A. Trogdon and D. D. Joseph. The stick-slip problem for a round jet I, large surface tension. Rheologica Acta, 19 (1980), 404-420.

[17] A. Trogdon and D. D. Joseph. The stick-slip problem for a round jet II, small surface tension. Rheologica Acta, 20 (1981), 1-13. 